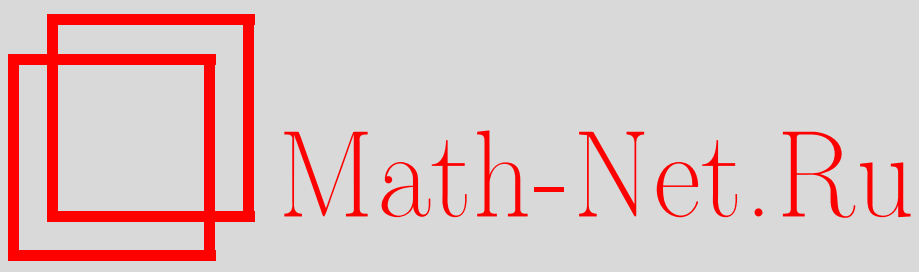

Вик. С. Куликов, Старые и новый примеры поверхностей общего типа с $p_{g}=0$, Изв. РАН. Сер. матем., 2004, том 68, выпуск 5, 123-170

DOI: https://doi.org/10.4213/im505

Использование Общероссийского математического портала Math-Net.Ru подразумевает, что вы прочитали и согласны с пользовательским соглашением

http://www . mathnet.ru/rus/agreement

Параметры загрузки:

IP : 54.237 .59 .107

26 апреля 2023 г., 03:03:24 
УДК 512.7

Вик. С. Куликов

\title{
Старые и новый примеры поверхностей общего типа с $p_{g}=0$
}

\begin{abstract}
Рассмотрены поверхности общего типа геометрического рода $p_{g}=0$, которые могут быть заданы как накрытия Галуа проективной плоскости, имеющие группу Галуа $G=(\mathbb{Z} / q \mathbb{Z})^{k}$, где $k \geqslant 2$ и $q$ - простое число, и разветвленные вдоль некоторой конфигурации прямых. В качестве таких накрытий можно получить классическую поверхность Годо, поверхности Кампеделли, поверхности Бурниа и новую поверхность $X$ с инвариантами $K_{X}^{2}=6$ и $(\mathbb{Z} / 3 \mathbb{Z})^{3} \subset \operatorname{Tors}(X)$. Доказано, что группа автоморфизмов общей поверхности Кампеделли изоморфна группе $(\mathbb{Z} / 2 \mathbb{Z})^{3}$. Описаны неприводимые компоненты пространства модулей поверхностей, содержащих поверхности Бурниа. Доказано, что поверхность Бурниа $S$ с $K_{S}^{2}=2$ имеет группу кручения $\operatorname{Tors}(S) \simeq(\mathbb{Z} / 2 \mathbb{Z})^{3}$ (следовательно, она принадлежит семейству поверхностей Кампеделли), т. е. соответствующее утверждение в статьях [9], [4], а также в книге [1, с. 237] о группе кручения поверхности Бурниа $S$ с $K_{S}^{2}=2$ является не верным.
\end{abstract}

Библиография: 10 наименований.

\section{Введение}

Как известно, индекс самопересечения канонического класса поверхности общего типа геометрического рода $p_{g}=0$ может принимать значения $K^{2}=1, \ldots, 9$, и в прошлом столетии было доказано сушествование таких поверхностей для всех возможных значений $K^{2}$. Тем не менее, наши знания о поверхностях общего типа геометрического рода, равного нулю, далеко не полны. В частности, до сих пор полностью не описаны пространства модулей таких поверхностей. Более того, не известен список всех возможных абелевых групп, которые могут быть реализованы как группы кручения этих поверхностей.

В настоящей статье исследованы поверхности общего типа с $p_{g}=0$, которые могут быть заданы как накрытия Галуа проективной плоскости с групой Галуа $G=(\mathbb{Z} / q \mathbb{Z})^{k}$, где $k \geqslant 2$ и $q$ является простым числом, разветвленные над некоторой конфигурацией прямых. В частности, классическая поверхность Годо [5], поверхности Кампеделли [3], [8], поверхности Бурниа [2] и новая поверхность $X$ с $K_{X}^{2}=6$ и $(\mathbb{Z} / 3 \mathbb{Z})^{3} \subset \operatorname{Tors}(X)=\operatorname{Tors} H_{1}(X, \mathbb{Z})=\operatorname{Tors} H^{2}(X, \mathbb{Z})$ могут быть получены как такие накрытия. Доказано, что группа автоморфизмов общей поверхности Кампеделли изоморфна группе $(\mathbb{Z} / 2 \mathbb{Z})^{3}$. Показано, что поверхность Бурниа $S$ с $K_{S}^{2}=2$ имеет группу кручения $\operatorname{Tors}(S) \simeq(\mathbb{Z} / 2 \mathbb{Z})^{3}$ (следовательно, она принадлежит семейству поверхностей Кампеделли; см. предложение 4.24), и тем самым

Работа частично поддержана РФФИ (грант № 02-01-00786). 
соответствующее утверждение в [9], [4] и в [1, с. 237] о группе кручения поверхности Бурниа $S$ с $K_{S}^{2}=2$ является не верным.

Описание неприводимых компонент пространства модулей поверхностей, содержаших поверхности Бурниа, дает следующая диаграмма:

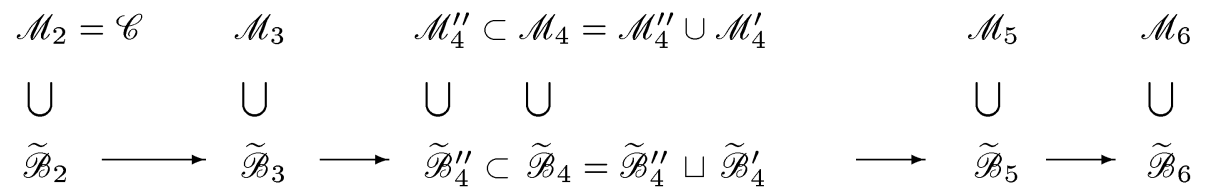

в которой через $\mathscr{M}_{k}$ обозначено объединение неприводимых компонент пространства модулей поверхностей с $p_{g}=0$ и $K^{2}=k$, содержаших поверхности Бурниа, и через $\mathscr{C}$ - пространство модулей поверхностей Кампеделли. Точки подмногообразий $\widetilde{\mathscr{B}}_{k}$ пространств $\mathscr{M}_{k}$ соответствуют поверхностям Бурниа. Многообразия $\widetilde{\mathscr{B}}_{k}$ при $k \neq 4$ унирациональны и $\widetilde{\mathscr{B}}_{4}$ состоит из двух рациональных поверхностей (точки неприводимой компоненты $\widetilde{\mathscr{B}}_{4}^{\prime \prime}$ параметризуют поверхности Бурниа с $K^{2}=4$, содержащие $(-2)$-кривую), $\widetilde{\mathscr{B}}_{2}$ состоит из одной точки, $\widetilde{\mathscr{B}}_{3}$ является рациональной кривой, $\operatorname{dim} \widetilde{\mathscr{B}}_{5}=3$ и $\operatorname{dim} \widetilde{\mathscr{B}}_{6}=4$. Подмногообразия $\widetilde{\mathscr{B}}_{k}$ всюду плотны в $\mathscr{M}_{k}$ при $k \geqslant 4, \operatorname{dim} \mathscr{M}_{3}=4$, и, как известно (см. [8]), $\mathscr{C}$ также унирационально, $\operatorname{dim} \mathscr{C}=6$. Стрелки в диаграмме показывают примыкания компонент (например, $\widetilde{\mathscr{B}}_{3} \rightarrow \widetilde{\mathscr{B}}_{4}^{\prime \prime}$ означает, что поверхности Бурниа с $K^{2}=3$ являются вырождениями поверхностей Бурниа с $K^{2}=4$, имеющих $(-2)$-кривые). Отметим, что аналогичный результат о компоненте $\widetilde{\mathscr{B}}_{6}$ был получен в [10] с использованием другой техники.

Статья организована следуюшим образом. В $\oint 1$ напоминаются основные факты о накрытиях Галуа $g: Y \rightarrow \mathbb{P}^{2}$ плоскости $\mathbb{P}^{2}$ с группой Галуа $G=(\mathbb{Z} / q \mathbb{Z})^{k}$, разветвленные вдоль конфигурации прямых $\bar{L} \subset \mathbb{P}^{2}$, и показано, как получить разрешение $X$ особых точек многообразия $Y$ в терминах особых точек конфигурации $\bar{L}$. Затем эти результаты используются в $\S 2$ для вычисления $K_{X}^{2}$ и топологической эйлеровой характеристики $e(X)$, в 33 напоминается алгоритм вычисления геометрического рода поверхности $X$. В $\S 4$ рассмотрены упомянутые выше примеры и приведены доказательства основных результатов.

Автор выражает искреннюю благодарность Университету г. Падуя (Италия), во время пребывания в котором была начата работа над данной статьей.

\section{§ 1. Абелевы накрытия плоскости, разветвленные в конфигурациях прямых}

По определению накрытие Галуа гладкого алгебраического многообразия $Y$ это конечный морфизм $f: X \rightarrow Y$ нормального алгебраического многообразия $X$ на $Y$ такой, что вложение полей рациональных функций $\mathbb{C}(Y) \subset \mathbb{C}(X)$, индуцированное морфизмом $f$, является расширением Галуа. Как хорошо известно, конечный морфизм $f: X \rightarrow Y$ является накрытием Галуа с группой Галуа $G$ тогда и только тогда, когда $G$ совпадает с групой накрывающих преобразований, действующих транзитивно на каждом слое морфизма $f$. Кроме того, конечное разветвленное накрытие является накрытием Галуа тогда и только тогда, когда неразветвленная часть этого накрытия (т. е. ограничение морфизма на дополнения 
к ветвлениям внизу и наверху) является накрытием Галуа. Более того, морфизм накрытий Галуа из неразветвленной части одного разветвленного накрытия в неразветвленную часть другого может быть продолжен до морфизма разветвленных накрытий, если задано продолжение морфизма накрываемых многообразий в точки ветвления накрытий. В частности, разветвленное накрытие Галуа определяется однозначно с точностью до изоморфизма своей неразветвленной частью. Напомним также, что неразветвленное накрытие является накрытием Галуа с группой Галуа $G$ тогда и только тогда, когда это топологическое накрытие ассоциировано с эпиморфизмом из фундаментальной групшы накрываемого многообразия в $G$ и, в частности, накрытия Галуа с абелевой группой Галуа $G$ взаимно однозначно соответствуют эпиморфизмам из первой группы гомологий с целыми коэффициентами накрываемого многообразия в $G$. Все перечисленные свойства накрытий Галуа являются хорошо известными, и наиболее нетривиальная часть из них может быть получена, например, из теоремы Грауэрта-Ремерта [6].

Далее мы будем иметь дело только с накрытиями комплексной проективной плоскости $\mathbb{P}^{2}$, разветвленными вдоль некоторой конфигурации прямых $\bar{L}=$ $L_{1} \cup \cdots \cup L_{n}$. Простые петли $\lambda_{i}, 1 \leqslant i \leqslant n$, вокруг прямых $L_{i}$ порождают группу $H_{1}\left(\mathbb{P}^{2} \backslash \bar{L}, \mathbb{Z}\right) \simeq \mathbb{Z}^{n-1}$. Они удовлетворяют соотношению

$$
\lambda_{1}+\cdots+\lambda_{n}=0 .
$$

Подобно общим абелевым накрытиям Галуа, накрытие Галуа $g: Y \rightarrow \mathbb{P}^{2}$ с абелевой группой Галуа $G$, разветвленное вдоль $\bar{L}$, однозначно определяется некоторым эпиморфизмом $\varphi: H_{1}\left(\mathbb{P}^{2} \backslash \bar{L}, \mathbb{Z}\right) \rightarrow G$ и существует для любого такого эпиморфизма. Накрытие $g$ разветвлено вдоль прямой $L_{i} \subset \bar{L}$ тогда и только тогда, когда $\varphi\left(\lambda_{i}\right) \neq 0$ и, более того, индекс ветвления морфизма $g$ вдоль $L_{i}$ совпадает с порядком элемента $\varphi\left(\lambda_{i}\right)$ в группе $G$.

Поскольку $H_{1}\left(\mathbb{P}^{2} \backslash \bar{L}, \mathbb{Z}\right) \simeq \mathbb{Z}^{n-1}$, то существует, в частности, универсальное накрытие $g_{u(m)}: Y_{u(m)} \rightarrow \mathbb{P}^{2}$, соответствуюшее естественному эпиморфизму

$$
\bar{\varphi}: H_{1}\left(\mathbb{P}^{2} \backslash \bar{L}, \mathbb{Z}\right) \rightarrow H_{1}\left(\mathbb{P}^{2} \backslash \bar{L}, \mathbb{Z} / m \mathbb{Z}\right)=H_{1}\left(\mathbb{P}^{2} \backslash \bar{L}, \mathbb{Z}\right) \otimes(\mathbb{Z} / m \mathbb{Z}) .
$$

Простейшим примером такого накрытия является следующий

Пример. Пусть конфигурация $\bar{L}=L_{0}+L_{1}+L_{2} \subset \mathbb{P}^{2}$ задана уравнением $x_{0} x_{1} x_{2}=0$, где $\left(x_{0}: x_{1}: x_{2}\right)$ - однородные координаты в $\mathbb{P}^{2}$. Легко видеть, что накрытие $g_{u(m)}: \mathbb{P}^{2} \rightarrow \mathbb{P}^{2}$, заданное уравнениями $y_{i}=x_{i}^{m}, i=0,1,2$, ассоциировано с эпиморфизмом

$$
\bar{\varphi}: H_{1}\left(\mathbb{P}^{2} \backslash \bar{L}, \mathbb{Z}\right) \simeq \mathbb{Z}^{2} \rightarrow(\mathbb{Z} / m \mathbb{Z})^{2} .
$$

Следующее утверждение является непосредственным следствием общих результатов о разветвленных накрытиях, упомянутых в начале этого параграфа.

ПредлОЖениЕ 1.1. Если $g: Y \rightarrow \mathbb{P}^{2}$ является накрытием Галуа с группой Галуа $G \simeq(\mathbb{Z} / m \mathbb{Z})^{k}$, разветвленным вдоль $\bar{L}$, то $k \leqslant n-1$ и для любого эпиморфизма $H_{1}\left(\mathbb{P}^{2} \backslash \bar{L}\right) \rightarrow G$ существует однозначно определенное накрытие Галуа $h: Y_{u(m)} \rightarrow Y$, индуцированное этим әпиморфизмом и такое, что $g_{u(m)}=g \circ h$. 
Далее ограничимся рассмотрением только накрытий Галуа с группой Галуа $G \simeq(\mathbb{Z} / q \mathbb{Z})^{k}$ и будем строить их так, как это описано в предложении 1.1.

Положим

$$
G_{u}=\left\{\bar{\gamma}=\left(\gamma_{1}, \ldots, \gamma_{n-1}\right) \mid \gamma_{i} \in \mathbb{Z} / q \mathbb{Z}\right\} \simeq(\mathbb{Z} / q \mathbb{Z})^{n-1},
$$

и пусть $\check{G}_{u} \simeq(\mathbb{Z} / q \mathbb{Z})^{n-1}$ - двойственная (как векторное пространство над $\mathbb{Z} / q \mathbb{Z}$ ) группа. Для элементов $\bar{\gamma}=\left(\gamma_{1}, \ldots, \gamma_{n-1}\right) \in G_{u}$ и $\bar{a}=\left(a_{1}, \ldots, a_{n-1}\right) \in \check{G}_{u}$ спаривание $(\bar{\gamma}, \bar{a})$ задается формулой

$$
(\bar{\gamma}, \bar{a})=\sum_{j=1}^{n-1} \gamma_{j} a_{j} \in \mathbb{Z} / q \mathbb{Z} .
$$

Не ограничивая общности, можно предполагать, что универсальное накрытие $g_{u}: Y_{u} \rightarrow \mathbb{P}^{2}$ ассоциировано с эпиморфизмом $\bar{\varphi}: H_{1}\left(\mathbb{P}^{2} \backslash \bar{L}, \mathbb{Z}\right) \rightarrow G_{u}$, отображающим $\lambda_{n}$ в $(q-1, \ldots, q-1)$ и $\lambda_{i}$ с $1 \leqslant i \leqslant n-1$ в $(0, \ldots, 0,1,0, \ldots, 0)$ с единицей, стоящей на $i$-м месте. Выберем, кроме того, прямую $L_{\infty} \subset \mathbb{P}^{2}$, находящуюся в общем положении с конфигурацией $\bar{L}$, и аффинные координаты $(x, y)$ в $\mathbb{C}^{2}=\mathbb{P}^{2} \backslash L_{\infty}$. Пусть $l_{i}(x, y)=0$ - линейное уравнение прямой $L_{i} \cap \mathbb{C}^{2}$. Положим $z_{i}=\left(l_{i} l_{n}^{q-1}\right)^{1 / q}$, $1 \leqslant i \leqslant n-1$. Тогда поле рациональных функций

$$
K_{u}=\mathbb{C}\left(Y_{u}\right)=\mathbb{C}\left(x, y, z_{1}, \ldots, z_{n-1}\right)
$$

на нормальном многообразии $Y_{u}$ является расширением степени $q^{n-1}$ поля рациональных функций $K=\mathbb{C}(x, y)$ на плоскости $\mathbb{P}^{2}$. (Другими словами, прообраз дополнения $\mathbb{P}^{2} \backslash L_{\infty}$ в многообразии $Y_{u}$ естественным образом изоморфен нормализации аффинного подмногообразия в $\mathbb{C}^{n+1}$, заданного в координатах $x, y, z_{1}, \ldots, z_{n-1}$ уравнениями $z_{1}^{q}=l_{1} l_{n}^{q-1}, \ldots, z_{n-1}^{q}=l_{n-1} l_{n}^{q-1}$.)

Для мультииндекса $\bar{a}=\left(a_{1}, \ldots, a_{n-1}\right), 0 \leqslant a_{i} \leqslant q-1$, положим

$$
z^{\bar{a}}=\prod_{i=1}^{n-1} z_{i}^{a_{i}}
$$

Действие элементов $\bar{\gamma}=\left(\gamma_{1}, \ldots, \gamma_{n-1}\right) \in G_{u}$ на элементах поля $K_{u}$ задается формулой

$$
\bar{\gamma}\left(z^{\bar{a}}\right)=\mu^{(\bar{\gamma}, \bar{a})} z^{\bar{a}}
$$

где $\mu=e^{2 \pi \sqrt{-1} / q}-$ корень из единищы $q$-й степени. Таким образом, группа Галуа $\operatorname{Gal}\left(K_{u} / \mathbb{C}[x, y]\right)=G_{u}$ и поле

$$
K_{u}=\bigoplus_{0 \leqslant a_{i} \leqslant q-1} \mathbb{C}(x, y) z^{\bar{a}}
$$

раскладывается как векторное пространство над $\mathbb{C}(x, y)$ в конечную прямую сумму представлений групшы $G_{u}$ степени 1. 
Пусть $\varphi: H_{1}\left(\mathbb{P}^{2} \backslash \bar{L}, \mathbb{Z}\right) \rightarrow(\mathbb{Z} / q \mathbb{Z})^{k}$ - эпиморфизм, заданньй формулами $\varphi\left(\lambda_{i}\right)=$ $\left(a_{i, 1}, \ldots, a_{i, k}\right)$, где $a_{1, j}+\cdots+a_{n, j} \equiv 0 \bmod q$ для $j=1, \ldots, k$, и пусть $g$ : $Y \rightarrow \mathbb{P}^{2}$ - соответствующее накрытие Галуа. Эпиморфизм $\varphi$ индуцирует эпиморфизм $\psi: G_{u} \rightarrow G$. Согласно предложению 1.1 существует однозначно определенное накрытие Галуа $h: Y_{u} \rightarrow Y$. Оно задает вложение $h^{*}: \mathbb{C}(Y) \rightarrow K_{u}$ поля функций $\mathbb{C}(Y)$ многообразия $Y$ в поле функций $K_{u}=\mathbb{C}\left(Y_{u}\right)$.

Поскольку $\operatorname{Gal}\left(K_{u} / h^{*}(\mathbb{C}(Y))\right)=\operatorname{ker} \psi$, то, очевидно, поле $h^{*}(\mathbb{C}(Y))$ совпадает с подполем $K_{\varphi}=\mathbb{C}\left(x, y, w_{1}, \ldots, w_{k}\right)$ поля $K_{u}$, где $w_{j}=z_{1}^{a_{1, j}} \ldots z_{n-1}^{a_{n-1, j}}$, и

$$
\operatorname{Gal}\left(K_{u} / K_{\varphi}\right)=\left\{\left(\gamma_{1}, \ldots, \gamma_{n-1}\right) \in G \mid \sum_{i=1}^{n-1} a_{i, j} \gamma_{i} \equiv 0 \quad \bmod q, 1 \leqslant j \leqslant k\right\}
$$

По построению поверхность $Y$ является нормальньм многообразием с изолированными особыми точками. Особые точки многообразия $Y$ могут возникать только над $r$-кратными точками конфигурации $\bar{L}$ при $r \geqslant 2$, т. е. над точками пересечения $r$ прямых $L_{i_{1}}, \ldots, L_{i_{r}}$.

Назовем два элемента группы $(\mathbb{Z} / q \mathbb{Z})^{k}$ линейно независимымми над $\mathbb{Z} / q \mathbb{Z}$, если они порождают в $(\mathbb{Z} / q \mathbb{Z})^{k}$ подгруппу, изоморфную $(\mathbb{Z} / q \mathbb{Z})^{2}$.

ЛЕмма 1.2. Пусть $p$ - двойная точка конфигурации $\bar{L}$, и пусть $\varphi\left(\lambda_{i_{1}}\right)$ и $\varphi\left(\lambda_{i_{2}}\right)$ являются линейно независимьми над $\mathbb{Z} / q \mathbb{Z}$ в группе $(\mathbb{Z} / q \mathbb{Z})^{k}$. Тогда поверхность $Y$ является неособой в каждой точке прообраза $g^{-1}(p)$.

ДокАЗАТЕЛЬСтво. Пусть $p=L_{i_{1}} \cap L_{i_{2}}$. Выберем малую окрестность $U$ точки $p$ в $\mathbb{P}^{2}$ и локальные координаты $u_{1}, u_{2}$ в $U$ так, что $U \simeq\left\{\left|u_{1}\right|^{2}+\left|u_{2}\right|^{2}<\varepsilon\right\}$ и $u_{j}=0$ является уравнением прямой $L_{i_{j}}$. Тогда

$$
H_{1}\left(U \backslash\left(L_{i_{1}} \cup L_{i_{2}}\right), \mathbb{Z}\right) \simeq \mathbb{Z} \oplus \mathbb{Z}
$$

В каждой точке $\tilde{p} \in g^{-1}(p)$ росток $V \rightarrow U$ накрытия $Y \rightarrow \mathbb{P}^{2}$ является $G^{\prime}$-накрытием, где $G^{\prime}$ - это образ группы $H_{1}\left(U \backslash\left(L_{i_{1}} \cup L_{i_{2}}\right), \mathbb{Z}\right)$ при композиции $\varphi \circ i_{*}$ эпиморфизма $\varphi$ и гомоморфизма $i_{*}: H_{1}\left(U \backslash\left(L_{i_{1}} \cup L_{i_{2}}\right), \mathbb{Z}\right) \rightarrow H_{1}\left(\mathbb{P}^{2} \backslash \bar{L}, \mathbb{Z}\right)$, индуцированного вложением многообразий. Более того, это $G^{\prime}$-накрытие определяется однозначно гомоморфизмом $\varphi \circ i_{*}$. Отождествляя $\varphi\left(\lambda_{i_{1}}\right), \varphi\left(\lambda_{i_{2}}\right)$ со стандартными порождающими группы $(\mathbb{Z} / q \mathbb{Z})^{2}$, получаем изоморфизм между $V \rightarrow U$ и накрытием, заданным уравнениями $z_{1}^{q}=u_{1}, z_{2}^{q}=u_{2}$. Следовательно, $V$ является неособым многообразием.

В примерах, которые будут рассмотрены ниже, чтобы разрешить особые точки многообразия $Y$ над $r$-кратными точками конфигурации $\bar{L}$ при $r \geqslant 3$, достаточно раздуть все эти точки. Пусть $\sigma: \widetilde{\mathbb{P}}^{2} \rightarrow \mathbb{P}^{2}$ - раздутие этих точек, $L_{i}^{\prime}-$ собственньй прообраз прямой $L_{i}, E_{p}=\sigma^{-1}(p)$ - рациональная кривая, являющаяся прообразом $r$-кратной точки $p$, и $\varepsilon_{p} \in H_{1}\left(\widetilde{\mathbb{P}}^{2} \backslash \sigma^{-1}(\bar{L}), \mathbb{Z}\right)=H_{1}\left(\mathbb{P}^{2} \backslash \bar{L}, \mathbb{Z}\right)$ - простая петля вокруг кривой $E_{p}$.

Композиция отождествления $H_{1}\left(\widetilde{\mathbb{P}}^{2} \backslash \sigma^{-1}(\bar{L}), \mathbb{Z}\right)=H_{1}\left(\mathbb{P}^{2} \backslash \bar{L}, \mathbb{Z}\right)$ и эпиморфизма $\varphi$ является эпиморфизмом $\varphi: H_{1}\left(\widetilde{\mathbb{P}}^{2} \backslash \sigma^{-1}(\bar{L}), \mathbb{Z}\right) \rightarrow(\mathbb{Z} / q \mathbb{Z})^{k}$. Рассмотрим соответствующее накрытие Галуа $f: X \rightarrow \widetilde{\mathbb{P}}^{2}$. 
Лемма 1.3. Пусть $p=L_{i_{1}} \cap \cdots \cap L_{i_{r}}-r$-кратная точка конфигурации $\bar{L}$. Тогда $\varepsilon_{p}=\lambda_{i_{1}}+\cdots+\lambda_{i_{r}}$.

ДокАЗАТЕЛЬСТво. Чтобы проверить требуемое равенство, достаточно рассмотреть обший пучок прямых, проходящих через точку $p$.

ЛЕмма 1.4. Если для каждой $r$-кратной точки $p=L_{i_{1}} \cap \cdots \cap L_{i_{r}}$ конфигурачии $\bar{L}$ при $r \geqslant 3$ либо пары әлементов $\varphi\left(\varepsilon_{p}\right)$ и $\varphi\left(\lambda_{i_{j}}\right), \quad 1 \leqslant j \leqslant r$, являются линейно независимыми над $\mathbb{Z} / q \mathbb{Z}$ в $(\mathbb{Z} / q \mathbb{Z})^{k}$, либо $\varphi\left(\varepsilon_{p}\right)=0$, то $X$ является неособой поверхностью.

ДоКАЗАТЕЛЬСТВо следует из лемм 1.2 и 1.3.

Пусть $p_{1}, \ldots, p_{s}$ - множество $r$-кратных точек, $r \geqslant 2$, конфигурации прямых $\bar{L}$, и пусть $\varphi: H_{1}\left(\mathbb{P}^{2} \backslash \bar{L}, \mathbb{Z}\right) \rightarrow(\mathbb{Z} / q \mathbb{Z})^{k}-$ эпиморфизм, заданный формулами $\varphi\left(\lambda_{i}\right)=$ $\left(a_{i, 1}, \ldots, a_{i, k}\right)$, где $a_{1, j}+\cdots+a_{n, j} \equiv 0 \bmod q$ для всех $j=1, \ldots, k$. Предположим, что все особые точки конфигурации $\bar{L}$ являются $\varphi$-хорошими точками, т. е. для всех $r$-кратных точек $p_{i_{1}, \ldots, i_{r}}=L_{i_{1}} \cap \cdots \cap L_{i_{r}}$ конфигурашии $\bar{L}$ с $r \geqslant 2$ либо пары элементов $\varphi\left(\varepsilon_{p_{i_{1}}, \ldots, i_{r}}\right)$ и $\varphi\left(\lambda_{i_{j}}\right), 1 \leqslant j \leqslant r$, являются линейно независимыми над $\mathbb{Z} / q \mathbb{Z}$ в $(\mathbb{Z} / q \mathbb{Z})^{k}$, либо $\varphi\left(\varepsilon_{p_{i_{1}, \ldots, i_{r}}}\right)=0$. Скажем, что $r$-кратная точка $p_{i_{1}, \ldots, i_{r}}=L_{i_{1}} \cap \cdots \cap L_{i_{r}}$ не является точкой ветвления относительно $\varphi$, если $\varphi\left(\varepsilon_{p_{i_{1}, \ldots, i_{r}}}\right)=0$.

Пусть $\sigma: \widetilde{\mathbb{P}}^{2} \rightarrow \mathbb{P}^{2}$ - раздутие с центром во всех $r$-кратных точках с $r \geqslant 3$ и во всех двойных точках конфигурации $\bar{L}$, не являюшихся точками ветвления. Согласно лемме 1.4 полученная поверхность $X$ является разрешением особых точек поверхности $Y$ и накрытие $f$ может быть включено в следующую коммутативную диаграмму:

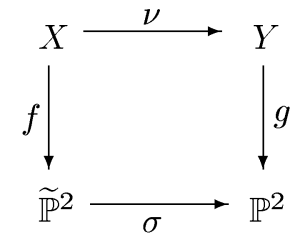

в которой $\nu$ - регулярное бирациональное отображение.

Пусть $N_{\varphi}$-множество всех $r$-кратных точек, не являюшихся точками ветвления относительно $\varphi$. Рассмотрим подпространство в $(\mathbb{Z} / q \mathbb{Z})^{n}=\left\{\left(x_{1}, \ldots, x_{n}\right) \mid x_{i} \in\right.$ $\mathbb{Z} / q \mathbb{Z}\}$ решений следующей системы линейных уравнений:

$$
\begin{aligned}
\sum_{i=1}^{n} x_{i} & =0 \\
\sum_{i \in\left\{i_{1}, \ldots, i_{r}\right\}} x_{i} & =0, \quad p_{i_{1}, \ldots, i_{r}} \in N_{\varphi} .
\end{aligned}
$$

Обозначим через $n_{\varphi}$ ранг этой линейной системы над $\mathbb{Z} / q \mathbb{Z}$. Имеем $k \leqslant k_{\varphi}=$ $n-n_{\varphi}$, так как ранг множества векторов

$$
A_{\varphi}=\left\{\left(a_{1, j}, \ldots, a_{n, j}\right)\right\}_{\{j=1, \ldots, k\}}
$$


равен $k$ и векторы из $A_{\varphi}$ удовлетворяют уравнениям (1.1). Расширим множество $A_{\varphi}$ до базиса $A_{u, \varphi}$ пространства решений над $\mathbb{Z} / q \mathbb{Z}$ линейной системы (1.1), добавив $k_{\varphi}-k$ векторов к множеству $A_{\varphi}$ :

$$
A_{u, \varphi}=\left\{\left(a_{1, j}, \ldots, a_{n, j}\right)\right\}_{\left\{j=1, \ldots, k_{\varphi}\right\}}
$$

и рассмотрим эпиморфизм

$$
\psi_{\varphi}: H_{1}\left(\mathbb{P}^{2} \backslash \bar{L}, \mathbb{Z}\right) \rightarrow G_{u, \varphi}=(\mathbb{Z} / q \mathbb{Z})^{k_{\varphi}},
$$

заданный формулами $\psi_{\varphi}\left(\lambda_{i}\right)=\left(a_{i, 1}, \ldots, a_{i, k_{\varphi}}\right)$. Очевидно, эпиморфизм $\varphi$ может быть разложен в композицию $\varphi=\eta \circ \psi_{\varphi}$, где $\eta:(\mathbb{Z} / q \mathbb{Z})^{k_{\varphi}} \rightarrow(\mathbb{Z} / q \mathbb{Z})^{k}-$ проекция на первые $k$ координат. Пусть $\bar{f}: \bar{X} \rightarrow \widetilde{\mathbb{P}}^{2}$ и $h_{u, \varphi}: \bar{X} \rightarrow X$ - накрытия Галуа, ассоциированные соответственно с $\psi_{\varphi}$ и $\eta$ (см. предложение 1.1). Заметим, что группа Галуа накрытия $h_{u, \varphi}$ изоморфна группе $(\mathbb{Z} / q \mathbb{Z})^{k_{\varphi}-k}$.

Группа $\operatorname{Tors}(X)=\operatorname{Tors} H_{1}(X, \mathbb{Z}) \simeq \operatorname{Tors} H^{2}(X, \mathbb{Z})$ называется группой круче-

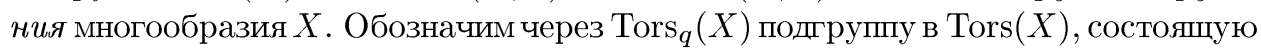
из элементов порядка $q$.

Из вышеизложенного следует

ПРеДЛОЖЕнИЕ 1.5. Пусть $f: X \rightarrow \widetilde{\mathbb{P}}^{2}$ - накрытие Галуа, ассочиированное с әпиморфизмом $\varphi: H_{1}\left(\mathbb{P}^{2} \backslash \bar{L}, \mathbb{Z}\right) \rightarrow(\mathbb{Z} / q \mathbb{Z})^{k}$, такое, что все особые точки конфигурации прямых $\bar{L}$ являются $\varphi$-хорошими. Предположим также, что $\varphi\left(\lambda_{i}\right) \neq 0$ для каждой прямой $L_{i} \subset \bar{L}$. Тогда $h_{u, \varphi}: \bar{X} \rightarrow X$ является неразветвленным накрытием.

СЛЕДСТВИЕ 1.6. Пусть накрытие $f: X \rightarrow \widetilde{\mathbb{P}}^{2}$ mо же, ито и в предложении 1.5. Если иррегулярность $q(X)=\operatorname{dim} H^{1}\left(X, \mathscr{O}_{X}\right)=0 u k_{\varphi}-k>0$, то әруппа q-кручения $\operatorname{Tors}_{q}(X)$ является нетривиальной. $B$ частности, существует вложение группы $\operatorname{ker} \eta \simeq(\mathbb{Z} / q \mathbb{Z})^{k_{\varphi}-k}$ в $\operatorname{Tors}_{q}(X)$.

\section{§ 2. Вычисление $K^{2}$ и эйлеровой характеристики}

Как и выше, пусть накрытие Галуа $g: Y \rightarrow \mathbb{P}^{2}$ с группой Галуа $G \simeq(\mathbb{Z} / q \mathbb{Z})^{k}$, разветвленное вдоль конфигурации прямых $\bar{L}=L_{1}+\cdots+L_{n}$, определено с помошью эпиморфизма $\varphi: H_{1}\left(\mathbb{P}^{2} \backslash \bar{L}, \mathbb{Z}\right) \rightarrow G$ такого, что $\varphi\left(\lambda_{i}\right) \neq 0$ для каждой $L_{i} \subset \bar{L}$. Предположим также, что все особые точки конфигурации $\bar{L}$ являются $\varphi$-хорошими. Обозначим через $\sigma: \widetilde{\mathbb{P}}^{2} \rightarrow \mathbb{P}^{2}$ композицию раздутий с центрами во всех $r$-кратных точках конфигурации $\bar{L} \mathrm{c} r \geqslant 3$ и во всех двойных точках, которые не являются точками ветвления относительно $\varphi$, и через $f: X \rightarrow \widetilde{\mathbb{P}}^{2}$ накрытие, индуцированное эпиморфизмом $\varphi$. Поскольку все особые точки конфигурации $\bar{L}$ являются $\varphi$-хорошими, то поверхность $X$ неособа.

Обозначим через $E_{p}=\sigma^{-1}(p)$ кривую, полученную в результате раздутия $r$-кратной точки $p, L_{i}^{\prime}=\sigma^{-1}\left(L_{i}\right)-$ собственный прообраз прямой $L_{i}, C_{i}=$ 
$f^{-1}\left(L_{i}^{\prime}\right)$ и $D_{p}=f^{-1}\left(E_{p}\right)$ - собственные прообразы кривых $L_{i}^{\prime}$ и $E_{p}$ соответственно. Пусть $T_{r}$ - это множество всех $r$-кратных точек конфигурации $\bar{L}$. Положим

$$
\begin{gathered}
T_{r}^{\prime}=\left\{p \in T_{r} \mid p \text { не является точкой ветвления для } \varphi\right\}, \\
T_{r}^{\prime \prime}=T_{r} \backslash T_{r}^{\prime}, \quad T^{\prime}=\bigcup_{r \geqslant 2} T_{r}^{\prime}, \quad T^{\prime \prime}=\bigcup_{r \geqslant 3} T_{r}^{\prime \prime}, \quad T=T^{\prime} \cup T^{\prime \prime} .
\end{gathered}
$$

Обозначим через $t_{r}^{\prime}=\# T_{r}^{\prime}$ (соответственно, $t_{r}^{\prime \prime}=\# T_{r}^{\prime \prime}$ ) число точек, принадлежащих множеству $T_{r}^{\prime}\left(\right.$ соответственно, $\left.T_{r}^{\prime \prime}\right)$, и положим $t_{r}=t_{r}^{\prime}+t_{r}^{\prime \prime}$. Заметим, что полньй прообраз $f^{*}\left(L_{i}^{\prime}\right)=q C_{i}$ для каждой прямой $L_{i} \subset \bar{L}$ и $f^{*}\left(E_{p}\right)=q D_{p}$ для каждой точки $p \in T^{\prime \prime}$.

ТЕорема 2.1. Индекс самопересечения $K_{X}^{2}$ канонического класса $K_{X}$ поверхности Х равен

$$
K_{X}^{2}=q^{k-2}\left[(q n-n-3 q)^{2}-\sum_{r \geqslant 2}(r q-q-r)^{2} t_{r}^{\prime}-\sum_{r \geqslant 3}(r q-2 q-r+1)^{2} t_{r}^{\prime \prime}\right] .
$$

ДокАЗАТЕЛЬСТво. Канонический класс поверхности $\widetilde{\mathbb{P}}^{2}$ равен

$$
K_{\widetilde{\mathbb{P}}^{2}}=-3 L+\sum_{p \in T} E_{p}
$$

где $L=\sigma^{*}\left(\mathbb{P}^{1}\right)$ - полный прообраз прямой $\mathbb{P}^{1} \subset \mathbb{P}^{2}$, и по формуле присоединения получим

$$
K_{X}=f^{*}\left(K_{\widetilde{\mathbb{P}}^{2}}\right)+(q-1)\left(\sum C_{i}+\sum_{p \in T^{\prime \prime}} D_{p}\right) .
$$

Кроме того, имеем

$$
\begin{aligned}
q \sum C_{i}= & f^{*}\left(n L-\sum_{r \geqslant 3} \sum_{p \in T_{r}} r E_{p}-2 \sum_{p \in T_{2}^{\prime}} E_{p}\right), \\
& q \sum_{p \in T^{\prime \prime}} D_{p}=f^{*}\left(\sum_{p \in T^{\prime \prime}} E_{p}\right) .
\end{aligned}
$$

Следовательно,

$$
\begin{aligned}
q K_{X}= & q f^{*}\left(K_{\widetilde{\mathbb{P}} 2}\right)+(q-1)\left(q \sum C_{i}+q \sum_{p \in T^{\prime \prime}} D_{p}\right) \\
= & q f^{*}\left(-3 L+\sum_{p \in T} E_{p}\right) \\
& +(q-1) f^{*}\left(n L-\sum_{r \geqslant 3} \sum_{p \in T_{r}} r E_{p}-2 \sum_{p \in T_{2}^{\prime}} E_{p}\right) \\
& +(q-1) f^{*}\left(\sum_{p \in T^{\prime \prime}} E_{p}\right)
\end{aligned}
$$


и, окончательно,

$$
\begin{aligned}
q K_{X}= & f^{*}\left((q n-n-3 q) L-\sum_{r \geqslant 2} \sum_{p \in T_{r}^{\prime}}(r q-q-r) E_{p}\right. \\
& \left.-\sum_{r \geqslant 3} \sum_{p \in T_{r}^{\prime \prime}}(r q-2 q-r+1) E_{p}\right) .
\end{aligned}
$$

Для каждого дивизора $D \in \operatorname{Pic} \widetilde{\mathbb{P}}^{2}$ имеем

$$
\left(f^{*}(D), f^{*}(D)\right)_{X}=\operatorname{deg} f \cdot(D, D)_{\widetilde{\mathbb{P}}^{2}}=q^{k}(D, D)_{\widetilde{\mathbb{P}}^{2}}
$$

и теорема следует из равенств $(L, L)_{\widetilde{\mathbb{P}}^{2}}=1,\left(L, E_{p}\right)_{\widetilde{\mathbb{P}}^{2}}=0$ и $\left(E_{p}, E_{p}\right)_{\widetilde{\mathbb{P}}^{2}}=-1$ для каждой кривой $E_{p}$.

Теорема 2.1 будет применяться в $\S 4$ в случае, когда конфигурации $\bar{L}$ и эпиморфизмы $\varphi$ удовлетворяют следуюшим условиям: $t_{2}^{\prime}=0, t_{4}^{\prime}=0$ и $t_{r}=0$ при $r \geqslant 5$. В этом случае формула (2.1) принимает следующий вид:

$$
K_{X}^{2}=q^{k-2}\left[(q n-n-3 q)^{2}-(2 q-3)^{2} t_{3}^{\prime}-(q-2)^{2} t_{3}^{\prime \prime}-(2 q-3)^{2} t_{4}^{\prime \prime}\right]
$$

Введем обозначение

$$
D_{K}=(q n-n-3 q) L-\sum_{r \geqslant 2} \sum_{p \in T_{r}^{\prime}}(r q-q-r) E_{p}-\sum_{r \geqslant 3} \sum_{p \in T_{r}^{\prime \prime}}(r q-2 q-r+1) E_{p}
$$

Поскольку $f$ является конечным накрытием Галуа, то имеет место следующее утверждение.

УТВЕРЖДЕНИЕ 2.2. Пусть дивизор $D_{K}$ является объемным, m. е. $D_{K}^{2}>0$. Тогда:

(i) поверхность $X$ не является минимальной моделью, если и только если существует неприводимая кривая $C \subset \widetilde{\mathbb{P}}^{2}$ такая, что $\left(D_{K}, C\right)_{\widetilde{\mathbb{P}}^{2}}<0$;

(ii) канонический класс поверхности $X$ не является обильным, если и только если существует неприводимая кривая $C \subset \widetilde{\mathbb{P}}^{2}$ такая, что $\left(D_{K}, C\right)_{\widetilde{\mathbb{P}}^{2}} \leqslant 0$.

ТЕОРема 2.3. Топологическая әйлерова характеристика поверхности $X$ выражсается формулой

$$
\begin{gathered}
e(X)=q^{k-2}\left(3 q^{2}-2 n\left(q^{2}-q\right)+q^{2} \sum_{r \geqslant 2} t_{r}^{\prime}+(q-1)^{2} t_{2}^{\prime \prime}\right. \\
\left.+\left((r-1)(q-1)^{2}+1\right) \sum_{r \geqslant 3} t_{r}^{\prime \prime}\right) .
\end{gathered}
$$


ДокАЗАТЕЛЬСтво. Обозначим через

$$
B=\sum_{i=1}^{n} L_{i}^{\prime}+\sum_{p \in T^{\prime \prime}} E_{p}
$$

кривую ветвления морфизма $f$. Легко видеть, что

$$
e(\operatorname{Sing} B)=\# \operatorname{Sing} B=t_{2}^{\prime \prime}+\sum_{r \geqslant 3} r t_{r}^{\prime \prime}
$$

где Sing $B$ - множество двойных точек кривой $B$.

Топологическая эйлерова характеристика кривой $B$ определяется следуюшим образом:

$$
e(B)=2\left(n+\sum_{r \geqslant 3} t_{r}^{\prime \prime}\right)-\# \operatorname{Sing} B=2 n-t_{2}^{\prime \prime}-\sum_{r \geqslant 3}(r-2) t_{r}^{\prime \prime},
$$

так как $B$ является дивизором с нормальными пересечениями и топологическая эйлерова характеристика каждой неприводимой компоненты кривой $B$ равна двум.

Топологическая эйлерова характеристика поверхности $\widetilde{\mathbb{P}}^{2}$ выражается формулой

$$
e\left(\widetilde{\mathbb{P}}^{2}\right)=3+\sum_{r \geqslant 2} t_{r}^{\prime}+\sum_{r \geqslant 3} t_{r}^{\prime \prime}
$$

Имеем

$$
\begin{aligned}
e(X) & =q^{k} e\left(\widetilde{\mathbb{P}}^{2} \backslash B\right)+q^{k-1} e(B \backslash \operatorname{Sing} B)+q^{k-2} e(\operatorname{Sing} B) \\
& =q^{k-2}\left(q^{2} e\left(\widetilde{\mathbb{P}}^{2}\right)-\left(q^{2}-q\right) e(B)-(q-1) e(\operatorname{Sing} B)\right) .
\end{aligned}
$$

Чтобы завершить доказательство, достаточно подставить соотношения (2.4)-(2.6) в $(2.7)$.

В случае конфигураций прямых $\bar{L}$ и эпиморфизмов $\varphi$, удовлетворяющих условиям $t_{2}^{\prime}=0, t_{4}^{\prime}=0$ и $t_{r}=0$ при $r \geqslant 5$, формула $(2.3)$ принимает следуюший вид:

$$
\begin{aligned}
e(X)= & q^{k-2}\left(3 q^{2}-2 n\left(q^{2}-q\right)+q^{2} t_{3}^{\prime}+(q-1)^{2} t_{2}^{\prime \prime}\right. \\
& \left.+\left(2(q-1)^{2}+1\right) t_{3}^{\prime \prime}+\left(3(q-1)^{2}+1\right) t_{4}^{\prime \prime}\right) .
\end{aligned}
$$




\section{§ 3. Вычисление геометрического рода}

Цель данного параграфа - объяснить общий алгоритм, которьй будет применен для вычисления геометрического рода. Фактически, если геометрический род накрытия вычислен, то тем самым вычислена и иррегулярность накрытия, так как их разность является топологическим инвариантом, равным согласно формуле Нетера $\frac{K_{X}^{2}+e(X)}{12}-1$. В процессе вычисления будет постоянно использоваться инвариантность геометрического рода при бирациональных преобразованиях, которая позволяет на каждом шаге использовать ту неособую бирациональную модель, которая более удобна для вычислений.

Алгоритм вычисления, который будет использоваться, не является новым, он содержится, например, в [7]. Напомним его основные шаги.

3.1. Редукция к циклическим накрытиям. Пусть $g: Y_{G} \rightarrow \mathbb{P}^{2}$-накрытие Галуа с абелевой группой Галуа $G=(\mathbb{Z} / q \mathbb{Z})^{k}$, разветвленное вдоль кривых $B_{1}, \ldots, B_{n} \subset \mathbb{P}^{2}$, где $Y_{G}$ - нормальная поверхность. Как и вьше, такое накрытие определяется эпиморфизмом $\varphi: H_{1}\left(\mathbb{P}^{2} \backslash \cup B_{i}\right) \rightarrow G$. Запишем его в виде

$$
\varphi\left(\gamma_{i}\right)=m_{1, i} \alpha_{1}+\cdots+m_{k, i} \alpha_{k}, \quad i=1, \ldots, n,
$$

где $\alpha_{j}$ - стандартные порождающие групшы $G=\bigoplus(\mathbb{Z} / q \mathbb{Z}), \quad \gamma_{i}$ - стандартные порождающие групшы $H_{1}\left(\mathbb{P}^{2} \backslash \cup B_{i}\right)$, двойственные к кривым $B_{i}$, и $m_{j, i} \in \mathbb{Z} / q \mathbb{Z}$, $0 \leqslant m_{j, i}<q,-$ координаты элементов $\varphi\left(\gamma_{i}\right)$ относительно базиса $\alpha_{j}$. В этих обозначениях поверхность $Y_{G}$ является нормализацией проективного замыкания аффинной поверхности $Y_{G, 0} \subset \mathbb{C}^{m+2}$, заданной уравнениями

$$
z_{j}^{q}=\prod_{i=1}^{n} h_{i}^{m_{j, i}}(x, y), \quad j=1, \ldots, k,
$$

где $h_{i}(x, y)$ - уравнения кривых $B_{i}$ в некоторой карте $\mathbb{C}^{2} \subset \mathbb{P}^{2}$.

Пусть $X_{G}$ - минимальное разрешение особенностей поверхности $Y_{G}$. Как известно, оно существует, единственно и действие групшы $G$ на $Y_{G}$ однозначно поднимается до регулярного действия на $X_{G}$.

Рассмотрим действие групшы $G$ на пространстве $H^{0}\left(X_{G}, \Omega_{X_{G}}^{2}\right)$ регулярных 2-форм. Оно задает разложение

$$
H^{0}\left(X_{G}, \Omega_{X_{G}}^{2}\right)=\bigoplus H_{\left(s_{1}, \ldots, s_{k}\right)}
$$

в прямую сумму собственных подпространств $H_{\left(s_{1}, \ldots, s_{k}\right)}$, где $\omega \in H_{\left(s_{1}, \ldots, s_{k}\right)}$ тогда и только тогда, когда $\alpha_{j}(\omega)=e^{2 \pi s_{j} \sqrt{-1} / q} \omega$ для $j=1, \ldots, k$. Пусть $H \subset G-$ некоторая подгруппа и $G_{1}=G / H$. Имеем следующую коммутативную диаграмму:

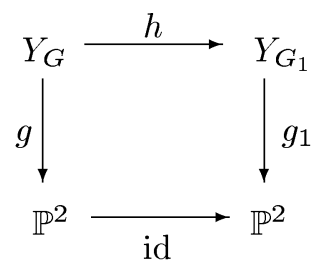


в которой $g_{1}: Y_{G_{1}} \rightarrow \mathbb{P}^{2}$ является накрытием Галуа, ассоциированным с эпиморфизмом $\varphi_{1}=i \circ \varphi$, где $i: G \rightarrow G_{1}=G / H$ - канонический эпиморфизм. Отображение $h$ индуцирует рациональное доминантное (т. е. имеющее всюду плотный образ) отображение $X_{G} \rightarrow X_{G_{1}}$, и это отображение, как любое доминантное отображение неособых многообразий, преобразует голоморфные 2-формы в голоморфные 2 -формы. Таким образом, подпространство $h^{*}\left(H^{0}\left(X_{G_{1}}, \Omega_{X_{G_{1}}}^{2}\right)\right) \subset H^{0}\left(X_{G}, \Omega_{X_{G}}^{2}\right)$ хорошо определено и совпадает с подпространством

$$
H^{0}\left(X_{G}, \Omega_{X_{G}}^{2}\right)^{H} \subset H^{0}\left(X_{G}, \Omega_{X_{G}}^{2}\right)
$$

элементов, неподвижных при действии группы $H$. С другой стороны, собственное подпространство $H_{\left(s_{1}, \ldots, s_{k}\right)}$ неподвижно при действии элемента $x_{1} \alpha_{1}+\cdots+x_{k} \alpha_{k}$ тогда и только тогда, когда $x_{1} s_{1}+\cdots+x_{k} s_{k}=0(\bmod q)$. Следовательно, сумма $\bigoplus H_{\left(\theta s_{1}, \ldots, \theta s_{k}\right)}$, взятая по $\theta \in \mathbb{Z} / q \mathbb{Z}$, совпадает с $H^{0}\left(\widetilde{X}_{G}, \Omega_{\widetilde{X}_{G}}^{2}\right)^{H}$, где

$$
H=\left\{x_{1} \alpha_{1}+\cdots+x_{k} \alpha_{k} \mid x_{1} s_{1}+\cdots+x_{k} s_{k}=0 \quad(\bmod q)\right\} .
$$

Таким образом, эта сумма изоморфна пространству $H^{0}\left(X_{G / H}, \Omega_{X_{G / H}}^{2}\right)$. Это рассуждение приводит к следующему утверждению.

ПРЕДЛОЖЕНИЕ 3.1. Геометрический род $p_{g}\left(X_{G}\right)=\operatorname{dim} H^{0}\left(X_{G}, \Omega_{X_{G}}^{2}\right)$ nоверхности $X_{G}$ выражается формулой

$$
p_{g}\left(X_{G}\right)=\sum_{H} p_{g}\left(X_{G / H}\right),
$$

где суммирование ведется по всем подгруппам $H$ группь $G$ ранга $\mathrm{rk} H=$ $\operatorname{rk} G-1$.

3.2. Циклические накрытия. Пусть теперь $G=\mathbb{Z} / q \mathbb{Z}$ является циклической группой. Чтобы вычислить $p_{g}\left(X_{G}\right)$, выберем однородные координаты $\left(x_{0}\right.$ : $\left.x_{1}: x_{2}\right)$ в $\mathbb{P}^{2}$ так, что прямая $x_{0}=0$ не принадлежит дивизору ветвления накрытия $g: Y_{G} \rightarrow \mathbb{P}^{2}$. Как и вьше, обозначим через $Y_{G}$ нормализацию проективного замыкания поверхности, заданной в $\mathbb{C}^{3}$ уравнением

$$
z^{p}=h(x, y),
$$

где $x=\frac{x_{1}}{x_{0}}, y=\frac{x_{2}}{x_{0}}$,

$$
h(x, y)=\prod_{i=1}^{n} h_{i}^{m_{i}}(x, y)
$$

и $h_{i}(x, y)$ - неприводимые уравнения в $\mathbb{C}^{2} \subset \mathbb{P}^{2}$ кривых $B_{i}$, составляющих дивизор ветвления, $0<m_{i}<q$. Заметим, что степень

$$
\operatorname{deg} h(x, y)=\sum m_{i} \operatorname{deg} h_{i}(x, y)=m q
$$

делится на $q$, так как прямая $x_{0}=0$ не принадлежит дивизору ветвления.

Легко видеть, что над картой $x_{1} \neq 0$ многообразие $Y_{G}$ совпадает с нормализацией поверхности, заданной в $\mathbb{C}^{3}$ уравнением

$$
w^{q}=\tilde{h}(u, v)
$$

где $u=\frac{1}{x}, v=\frac{y}{x}, \tilde{h}(u, v)=u^{m q} h\left(\frac{1}{u}, \frac{v}{u}\right)$ и $w=z u^{m}$. 
3.2.1. Условие регулярности над общей точкой базы. Рассмотрим форму

$$
\omega \in H^{0}\left(Y_{G} \backslash \operatorname{Sing} Y_{G}, \Omega_{Y_{G} \backslash \operatorname{Sing} Y_{G}}^{2}\right)
$$

и найдем критерий для ее регулярности вне дивизора ветвления и множества особых точек.

Над картой $x_{0} \neq 0$ форма $\omega$ может быть записана в виде

$$
\omega=\left(\sum_{j=0}^{q-1} z^{j} g_{j}(x, y)\right) \frac{d x \wedge d y}{z^{q-1}}
$$

где $g_{j}(x, y)$ - некоторые рациональные функции от $x$ и $y$. Форма

$$
\frac{d x \wedge d y}{z^{q-1}}
$$

не имеет ни нулей, ни полюсов вне прообраза дивизора ветвления. Следовательно, $\omega$ является регулярной в некоторой точке $(a, b) \notin \sum B_{i}$ тогда и только тогда, когда все $g_{j}(x, y)$ регулярны в этой точке.

Действительно, если некоторая функция $g_{j}(x, y)$ не регулярна в точке $(a, b)$, то сумма

$$
\sum_{j=0}^{q-1} z^{j} g_{j}(x, y)
$$

может быть записана в виде

$$
\frac{\sum_{j=0}^{q-1} z^{j} P_{j}(x, y)}{P_{q}(x, y)}
$$

где $P_{j}(x, y), j=0, \ldots, q,-$ такие многочлены, что $P_{j}(a, b) \neq 0$ для некоторого $j<q$ и $P_{q}(a, b)=0$. Следовательно,

$$
\sum_{j=0}^{q-1} z^{j} P_{j}(a, b)=0
$$

во всех $q$ точках, принадлежащих прообразу $f^{-1}(a, b)$, так как в противном случае $\omega$ не была бы регулярной формой над точкой $(a, b)$. С другой стороны, это невозможно, поскольку нетривиальный многочлен степени меньше $q$ не может иметь $q$ корней.

3.2.2. Условие регулярности над бесконечно удаленной прямой. Рассмотрим форму $\omega$ над картой $x_{1} \neq 0$,

$$
\omega=-\left(\sum_{j=0}^{q-1} w^{j} \frac{\tilde{g}_{j}(u, v)}{u^{j m+\operatorname{deg} g_{j}}}\right) \frac{1}{u^{3-m(q-1)}} \frac{d u \wedge d v}{w^{q-1}} .
$$

Аргументы, аналогичные приведенным выше, показывают, что критерий регулярности эквивалентен следующему ограничению на степени рациональных функций $g_{j}$ :

$$
\operatorname{deg} g_{j}(x, y) \leqslant(q-j-1) m-3 \text {. }
$$


3.2.3. Условие регулярности над неособой точкой кривой ветвления. Рассмотрим форму

$$
\omega=\left(\sum_{j=0}^{q-1} z^{j} g_{j}(x, y)\right) \frac{d x \wedge d y}{z^{q-1}}
$$

над неособой точкой $(a, b)$ одной из компонент $B_{i_{0}}$ кривой ветвления. Пусть $r_{j}-$ порядок нуля (или полюса, если $r_{j}<0$ ) функции $g_{j}$ вдоль кривой $B_{i_{0}}$, т. е. $g_{j}=$ $\bar{g}_{j} h_{i_{0}}^{r_{j}}$, где функция $\bar{g}_{j}$ не обрашается в нуль и не имеет полюсов вдоль кривой $B_{i_{0}}$. Так как точка $(a, b)$ является неособой точкой кривой $B$, мы можем предполагать, что $h_{i_{0}}(x, y)$ и некоторая функция $g(x, y)$ являются локальными аналитическими координатами в некоторой окрестности $U$ точки $(a, b)$ (обозначим их через $u$ и $v$ ). Таким образом, над $U$ поверхность $Y_{G}$ (после аналитической замены координат) изоморфна нормализации $Y_{G}$, ос поверхности в $\mathbb{C}^{3}$, заданной уравнением

$$
z^{q}=u^{k_{i_{0}}}
$$

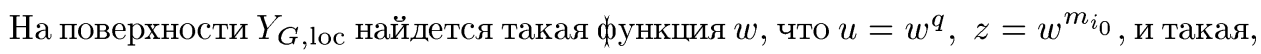

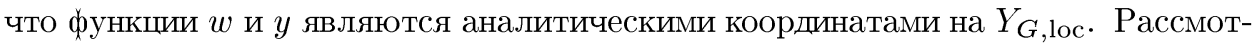
ренная выше дифференциальная 2-форма $\omega$ имеет следующий вид в новых координатах:

$$
\omega=\left(\sum_{j=0}^{q-1} w^{j m_{i_{0}}} \bar{g}_{j}(x, y) w^{q r_{j}}\right) \frac{q w^{q-1} d w \wedge d v}{w^{(q-1) m_{i_{0}}}} .
$$

Легко видеть, что

$$
j_{1} m_{i_{0}}+q r_{j_{1}}+q-1-(q-1) m_{i_{0}} \neq j_{2} m_{i_{0}}+q r_{j_{2}}+q-1-(q-1) m_{i_{0}}
$$

если $0<m_{i_{0}}<q, 0 \leqslant j_{1}, j_{2} \leqslant q-1$ и $j_{1} \neq j_{2}$. Следовательно, $\omega$ является регулярной формой над неособой точкой $(a, b)$, принадлежащей кривой $B_{i_{0}}$, тогда и только тогда, когда

$$
j m_{i_{0}}+q r_{j}+q-1-(q-1) m_{i_{0}} \geqslant 0
$$

для $0 \leqslant j \leqslant q-1$. Более того, если $\omega$ является регулярной формой над точками кривой $B_{i_{0}}$, то числа $r_{j}$ должны быть не меньше нуля, так как при $0<m_{i_{0}}<q$, $0 \leqslant j \leqslant q-1$ и $r_{j} \leqslant-1$ мы получили бы

$$
j m_{i_{0}}+q r_{j}+q-1-(q-1) m_{i_{0}}<0 .
$$

Отсюда следует, что если $\omega$ является регулярной формой, то все рациональные функции $g_{j}(x, y)$ являются регулярными всюду в $\mathbb{C}^{2}$ вне коразмерности два. Следовательно, $g_{j}(x, y)$ должны быть многочленами от $x$ и $y$. Более того, многочлены $g_{j}(x, y)$ должны делиться на $h_{i}^{r_{j}}(x, y)$, где $r_{j}$ - наименьшее целое неотрицательное число, удовлетворяющее неравенству

$$
q r_{j} \geqslant(q-j-1) m_{i}-q+1
$$


3.2.4. Условие регулярности над особой точкой кривой ветвления. Обозначим через $\nu: X_{G} \rightarrow Y_{G}$ минимальное разрешение особых точек поверхности $Y_{G}$ и через $E$ исключительный дивизор морфизма $\nu$. Пусть $\sigma: \widetilde{\mathbb{P}}^{2} \rightarrow \mathbb{P}^{2}-$ композиция $\sigma$-процессов с центрами в особых точках кривой $B$ (и их прообразах) такая, что $\sigma^{-1} \circ f \circ \nu\left(E_{i}\right)$ является кривой для каждой неприводимой компоненты $E_{i}$ дивизора $E$. Обозначим через $Z$ нормализацию поверхности $\widetilde{\mathbb{P}}^{2} \times_{\mathbb{P}^{2}} Y_{G}$, и пусть $g: X_{G} \rightarrow Z$ - бирациональное отображение, индуцированное морфизмами $\nu$ и $\sigma$. Из условия, наложенного на $\sigma$, следует, что для любой формы $\omega \in$ $H^{0}\left(Z \backslash \operatorname{Sing} Z, \Omega_{Z \backslash \operatorname{Sing} Z}^{2}\right)$ ее прообраз $g^{*}(\omega)$ является регулярной формой в общей точке каждой кривой $E_{i}$ и поэтому продолжается до регулярной формы на все $X_{G}$. Следовательно, пространство $H^{0}\left(X_{G}, \Omega_{X_{G}}^{2}\right)$ изоморфно пространству $H^{0}\left(Z \backslash \operatorname{Sing} Z, \Omega_{Z \backslash \operatorname{Sing} Z}^{2}\right)$.

Таким образом, необходимо рассмотреть 2-форму $\omega$, записанную в виде (3.1), и найти критерий ее регулярности в точках, принадлежаших $Z \backslash \operatorname{Sing} Z$. Это может быть сделано в результате последовательного применения выбранных вьше $\sigma$-процессов. Рассмотрим только первое раздутие, так как оно достаточно для вычисления геометрического рода в примерах, которые будут рассмотрены ниже.

Представим еще раз поверхность $Y_{G}$ как нормализацию поверхности, заданной уравнением

$$
z^{q}=h(x, y) .
$$

Обозначим через $r$ порядок нуля функции $h(x, y)$ в точке $(0,0), r=s q+c$, $0 \leqslant c<q$, и осушествим $\sigma$-процесс с центром в этой точке. В подходящей карте этот $\sigma$-процесс $\sigma: \mathbb{C}_{(u, v)}^{2} \rightarrow \mathbb{C}_{(x, y)}^{2}$ задается уравнениями $x=u, y=u v$. Нормализация $Z_{1}$ поверхности $Y_{G} \times_{\mathbb{C}_{(x, y)}^{2}} \mathbb{C}_{(u, v)}^{2}$ бирациональна нормализации поверхности, заданной уравнением

$$
w^{q}=u^{c} \bar{h}(u, v),
$$

где $w=z / u^{s}$ и $\bar{h}(u, v)=h(u, u v) / u^{r}$. Имеем

$$
\begin{aligned}
\omega & =\left(\sum_{j=0}^{q-1} z^{j} g_{j}(x, y)\right) \frac{d x \wedge d y}{z^{q-1}} \\
& =\left(\sum_{j=0}^{q-1} w^{j} \bar{g}_{j}(u, v) u^{s j+s_{j}+1-s(q-1)}\right) \frac{d u \wedge d v}{w^{q-1}},
\end{aligned}
$$

где $s_{j}$ - порядок нуля функции $g_{j}(x, y)$ в точке $(0,0)$. Применяя неравенство $(3.3)$, получаем необходимые условия для регулярности прообраза формы $\omega$ в общей точке исключительного дивизора: порядок нуля $s_{j}$ каждой функции $g_{j}(x, y)$ в особой точке кривой ветвления $B$ кратности $r$ является наименьшим целым числом, удовлетворяющим неравенству

$$
q s_{j} \geqslant(q-j-1) r-2 q+1 .
$$

Для вычисления геометрического рода каждой из поверхностей $X_{G_{i}}$ нужно найти все регулярные 2-формы, имеюшие такой же вид, как и в формуле (3.1), и которые удовлетворяют критериям (3.2)-(3.4).

Изложенное вьше приводит к следующему утверждению о $q$-листном циклическом накрытии, разветвленном вдоль конфигурации прямых $\bar{L}=L_{1}+\cdots+L_{n}$, в случаях $q=2$ и $q=3$. 
УТВЕРЖДЕНИЕ 3.2. Пусть $X$ - разрешение особенностей двулистного накрытия $g: Y \rightarrow \mathbb{P}^{2}$, разветвленного вдоль $\bar{L}=L_{1}+\cdots+L_{n}$. Обозначим через $T_{r}$ множество $r$-кратных точек конфигурации прямых $\bar{L}$ u $T=\cup T_{r}$. Тогда $n=2 m$ является четным числом и

$$
\begin{gathered}
p_{g}(X)=\operatorname{dim}_{\mathbb{C}}\left\{\bar{s} \in H^{0}\left(\mathbb{P}^{2}, \mathscr{O}_{\mathbb{P}^{2}}(m-3)\right) \mid \bar{s}\right. \text { имеет нуль порядка не меньие } \\
\left.\left\lceil\frac{r+1}{2}\right\rceil-2 \text { в точке } p \in T_{r} \text { для } \forall p \in T\right\},
\end{gathered}
$$

где $\left\lceil\frac{a}{b}\right\rceil$ - наименьшее челое число, которое больше или равно $\frac{a}{b}$.

УТВЕРЖДЕНИЕ 3.3. Пусть $X$ - разрешение особенностей трехлистного накрытия Галуа $g: Y \rightarrow \mathbb{P}^{2}$, в неоднородных координатах заданного уравнением

$$
z^{3}=\prod_{i=1}^{n} l_{i}(x, y)^{m_{i}},
$$

где $l_{i}(x, y)=0$ - уравнение прямой $L_{i} u$ каждое $m_{i}=1$ или 2 , а сумма $\sum m_{i}=3 m$ делится на 3 . Обозначим через $T_{r}$ мнохсество $r$-кратных точек дивизора $\prod_{i=1}^{n} l_{i}(x, y)^{m_{i}}=0, T=\bigcup T_{r} u \tilde{l}(x, y)=\prod_{i=1}^{n} l_{i}(x, y)^{m_{i}-1}$. Тогда

$$
p_{g}(X)=\operatorname{dim}_{\mathbb{C}} \mathscr{P}_{0}+\operatorname{dim}_{\mathbb{C}} \mathscr{P}_{1}
$$

$2 \partial e$

$$
\begin{aligned}
\mathscr{P}_{0}=\{s \in & H^{0}\left(\mathbb{P}^{2}, \mathscr{O}_{\mathbb{P}^{2}}\left(2 m-3-\sum\left(m_{i}-1\right)\right)\right) \mid \text { si имеет нуль } \\
& \text { порядка не меньие } \left.2\left\lceil\frac{r+1}{3}\right\rceil-2 \text { в точке } p \in T_{r} \text { для } \forall p \in T\right\}
\end{aligned}
$$

$u$

$$
\begin{gathered}
\mathscr{P}_{1}=\left\{\bar{s} \in H^{0}\left(\mathbb{P}^{2}, \mathscr{O}_{\mathbb{P}^{2}}(m-3)\right) \mid \bar{s} \text { имеет нуль порядка не меньие }\left\lceil\frac{r+1}{3}\right\rceil-2\right. \\
\text { в точке } \left.p \in T_{r} \text { для } \forall p \in T\right\} .
\end{gathered}
$$

\section{§ 4. Примеры}

4.1. Поверхности Кампеделли. Пусть $\bar{L}=L_{1}+\cdots+L_{7}$ - конфигурация прямых в $\mathbb{P}^{2}$, состояшая из семи прямых. Занумеруем их ненулевыми элементами $\alpha_{i} \in(\mathbb{Z} / 2 \mathbb{Z})^{3}$. Пусть конфигурация $\bar{L}$ не имеет $r$-кратных точек при $r \geqslant 4$, и если $\bar{L}$ имеет тройную точку $p_{\alpha_{1}, \alpha_{2}, \alpha_{3}}=L_{\alpha_{1}} \cap L_{\alpha_{2}} \cap L_{\alpha_{3}}$, то $\alpha_{1}+\alpha_{2}+\alpha_{3} \neq 0$. Такую конфигурацию прямых будем называть конфигурацией Кампеделли. Рассмотрим накрытие $g: Y \rightarrow \mathbb{P}^{2}$, индуцированное эпиморфизмом $\varphi: H_{1}\left(\mathbb{P}^{2} \backslash \bar{L}, \mathbb{Z}\right) \rightarrow G=$ $(\mathbb{Z} / 2 \mathbb{Z})^{3}$, заданным по правилу $\varphi\left(\lambda_{\alpha_{i}}\right)=\alpha_{i}$.

Поверхность $Y$ имеет особые точки, лежащие только над тройными точками $p_{\alpha_{1}, \alpha_{2}, \alpha_{3}}$. Чтобы разрешить их, раздуем все тройные точки и рассмотрим индуцированное накрытие Галуа $f: X \rightarrow \widetilde{\mathbb{P}}^{2}$. Пусть $\sigma: \widetilde{\mathbb{P}}^{2} \rightarrow \mathbb{P}^{2}$ - композиция раздутий с центрами в тройных точках. Построенную поверхность $X$ будем называть $n о$ верхностью Кампеделли. Обозначим через $E_{\alpha_{i}, \alpha_{j}, \alpha_{k}}=\sigma^{-1}\left(p_{\alpha_{i}, \alpha_{j}, \alpha_{k}}\right)$ исключительную кривую, лежащую над точкой $p_{\alpha_{i}, \alpha_{j}, \alpha_{k}}$. Поскольку $\alpha_{i}+\alpha_{j}+\alpha_{k} \neq 0$ для тройных точек, каждая кривая $E_{\alpha_{i}, \alpha_{j}, \alpha_{k}}$ является кривой ветвления накрытия $f$. Из леммы 1.4 следует, что $X$ является неособой поверхностью, так как элементы $\varphi\left(\varepsilon_{\alpha_{i}, \alpha_{j}, \alpha_{k}}\right)=\alpha_{i}+\alpha_{j}+\alpha_{k}$ и $\alpha_{i}$ (соответственно, $\alpha_{j}$ и $\left.\alpha_{k}\right)$ линейно независимы в $G$. Действительно, $\alpha_{i}+\alpha_{j}+\alpha_{k}$ и $\alpha_{i}$ являются линейно зависимыми в группе $G$ тогда и только тогда, когда $\alpha_{i}+\alpha_{j}+\alpha_{k}=\alpha_{i}$, т. е. когда $\alpha_{j}=\alpha_{k}$. 
ПРЕДЛОЖЕнИЕ 4.1. Построенные выше поверхности Кампеделли Х являются поверхностями общего типа с $K_{X}^{2}=2, p_{g}=0$ и $\operatorname{Tors}(X)=(\mathbb{Z} / 2 \mathbb{Z})^{3}$.

ДоКАЗАТЕЛЬСТво. Из утверждения 2.2 следует, что $2 K_{X}=\left|\tilde{f}^{*}(L)\right|$, где $L=$ $\sigma^{*}\left(\mathbb{P}^{1}\right)$ - полный прообраз прямой $\mathbb{P}^{1} \subset \mathbb{P}^{2}$. Следовательно, $X$ является поверхностью общего типа. Более того, она является минимальной моделью, так как $(L, C)_{\widetilde{\mathbb{P}} 2} \geqslant 0$ для каждой кривой $C \subset \widetilde{\mathbb{P}}^{2}$. Применяя $(2.2)$ и $(2.8)$, легко видеть, что $K_{X}^{2}=2$ и $e(X)=10$. Следовательно, согласно формуле Нётера арифметический род $p_{a}=1-q+p_{g}=1$. Как и выше, чтобы вычислить геометрический род $p_{g}$, достаточно вычислить геометрические роды семи циклических накрытий, соответствующих семи эпиморфизмам $\psi_{k}, \quad k=1, \ldots, 7$, групшы $G=(\mathbb{Z} / 2 \mathbb{Z})^{3}$ в циклическую группу $\mathbb{Z} / 2 \mathbb{Z}$. Легко видеть, что каждое из этих накрытий задается в неоднородных координатах уравнением вида $w_{k}^{2}=l_{\alpha_{i_{1}}} l_{\alpha_{i_{2}}} l_{\alpha_{i_{3}}} l_{\alpha_{i_{4}}}$, где $\alpha_{i_{1}}, \alpha_{i_{2}}, \alpha_{i_{3}}, \alpha_{i_{4}}$ - элементы группы $G$ такие, что $\psi_{k}\left(\alpha_{i_{j}}\right)=1$. Применяя утверждение 3.2 , легко проверить, что геометрический род каждого из этих накрытий равен нулю. Следовательно, поверхность $X$ имеет геометрический род $p_{g}=0$.

Чтобы показать, что $\operatorname{Tors}(X)=(\mathbb{Z} / 2 \mathbb{Z})^{3}$, рассмотрим универсальное накрытие $f_{u(2)}: X_{u(2)} \rightarrow \widetilde{\mathbb{P}}^{2}$, соответствующее эпиморфизму

$$
\bar{\varphi}: H_{1}\left(\mathbb{P}^{2} \backslash \bar{L}, \mathbb{Z}\right) \rightarrow H_{1}\left(\mathbb{P}^{2} \backslash \bar{L}, \mathbb{Z} / 2 \mathbb{Z}\right) \simeq(\mathbb{Z} / 2 \mathbb{Z})^{6},
$$

и накрытие $h: X_{u(2)} \rightarrow X$, соответствуюшее эпиморфизму $\psi:(\mathbb{Z} / 2 \mathbb{Z})^{6} \rightarrow G=$ $(\mathbb{Z} / 2 \mathbb{Z})^{3}$. Согласно предложению 1.5 и следствию 1.6 накрытие $h$ является неразветвленным и $(\mathbb{Z} / 2 \mathbb{Z})^{3} \subset \operatorname{Tors}(X)$. Следовательно $($ см. $[8]), \operatorname{Tors}(X)=(\mathbb{Z} / 2 \mathbb{Z})^{3}$.

Классическая поверхность Кампеделли $S$ (см. [3]) - это разрешение особенностей двойного накрытия $\tilde{g}: \widetilde{Y} \rightarrow \mathbb{P}^{2}$, разветвленного в объединении трех квадрик $Q_{1}, Q_{2}, Q_{3}$ и квартики $C_{4}$ в $\mathbb{P}^{2}$ таких, что кривая $B=Q_{1}+Q_{2}+Q_{3}+C_{4}$ имеет шесть особых точек типа $[3,3]$ (особая точка типа $[3,3]$ означает, что после раздутия с центром в особой точке собственный прообраз ростка кривой $B$ состоит из трех неприводимых гладких ветвей, пересекающихся трансверсально друг с другом).

Рассмотрим накрытие $f: X \rightarrow \widetilde{\mathbb{P}}^{2}$, разветвленное вдоль конфигурации Кампеделли $\bar{L}=\sum L_{\alpha_{i}}, \alpha_{i} \in(\mathbb{Z} / 2 \mathbb{Z})^{3} \backslash\{0\}$, имеющей три тройных точки. Эта конфигурация $\bar{L}$ изображена на рис. 1 . Покажем, что классическая поверхность Кампеделли $S$ изоморфна поверхности $X$.

Чтобы увидеть этот изоморфизм, рассмотрим раздутие $\sigma: \widetilde{\mathbb{P}}^{2} \rightarrow \mathbb{P}^{2}$ с центром в точках $p_{1}, p_{2}, p_{3}$ и обозначим через $E_{i}=\sigma^{-1}\left(p_{i}\right)$ исключительную кривую, лежащую над точкой $p_{i}$, а собственньй прообраз $\sigma^{-1}\left(L_{\alpha_{i}}\right) \subset \widetilde{\mathbb{P}}^{2}$ обозначим снова через $L_{\alpha_{i}}$. Можно проверить, что

$$
\varphi\left(\varepsilon_{i}\right)=(0,0,1)
$$

для $i=1,2,3$. Кривые $L_{(1,0,0)}, L_{(1,1,0)}, L_{(0,1,0)}$ в $\widetilde{\mathbb{P}}^{2}$ имеют индекс самопересечения, равньй -1 . Следовательно, их можно стянуть с помощью моноидального преобразования $\tau: \widetilde{\mathbb{P}}^{2} \rightarrow \mathbb{P}^{2}$ (композиция $\tau \circ \sigma^{-1}: \mathbb{P}^{2} \rightarrow \mathbb{P}^{2}$ является квадратичным преобразованием плоскости с центром в точках $\left.p_{1}, p_{2}, p_{3}\right)$. Кривые $L_{i}=\tau\left(E_{i}\right), \quad i=1,2,3$, и кривые $\tau\left(L_{(1,0,1)}\right), \tau\left(L_{(0,1,1)}\right), \tau\left(L_{(1,1,1)}\right)$ являются 


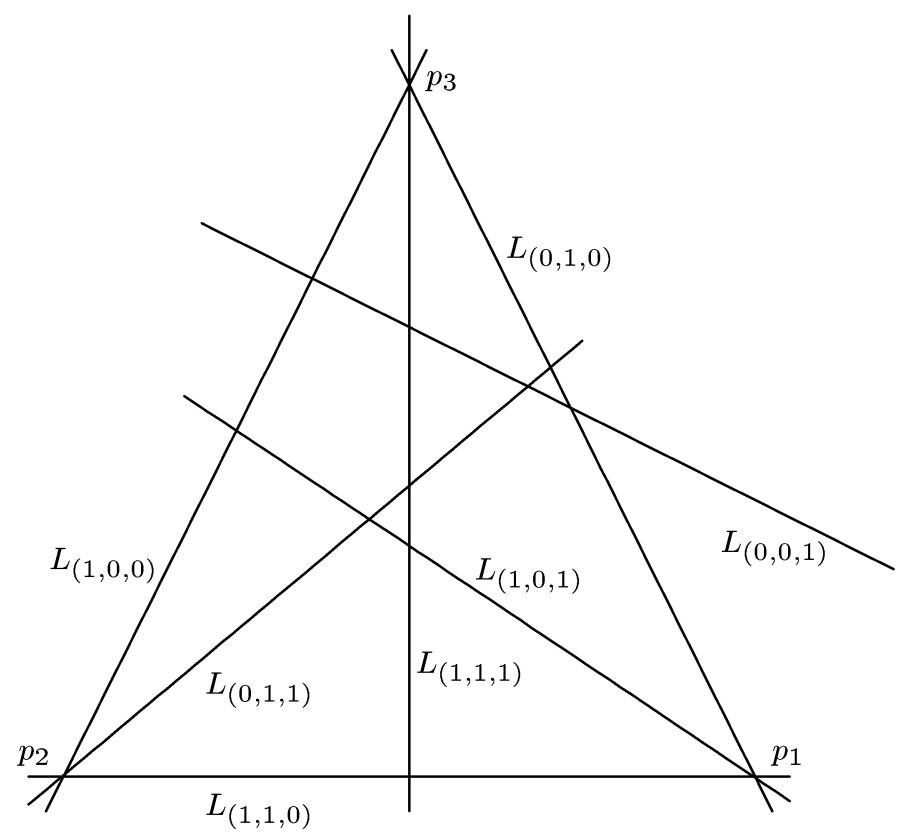

Рис. 1

прямыми, а $\tau\left(L_{(0,0,1)}\right)$ является коникой в $\mathbb{P}^{2}$. Имеем следующую коммутативную диаграмму:

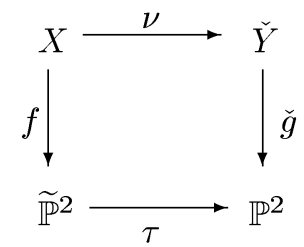

где $\check{Y}$ является нормальной поверхностью, $\nu: X \rightarrow \check{Y}$ - бирациональное отображение и $\check{g}: \check{Y} \rightarrow \mathbb{P}^{2}$ - накрытие Галуа, разветвленное вдоль прямых $L_{i}, i=1,2,3$, $\tau\left(L_{(1,0,1)}\right), \tau\left(L_{(0,1,1)}\right), \tau\left(L_{(1,1,1)}\right)$ и $\tau\left(L_{(0,0,1)}\right)$. Поскольку $\varphi\left(\lambda_{(0,0,1)}\right)=(0,0,1)$, то, принимая во внимание (4.1), легко видеть, что $\check{g}$ может быть разложено в композищию $\check{g}=g_{1} \circ \tilde{g}$, где $g_{1}: \mathbb{P}^{2} \rightarrow \mathbb{P}^{2}$ является накрытием Галуа с группой Галуа $G_{1}=(\mathbb{Z} / 2 \mathbb{Z})^{2}$, разветвленное вдоль прямых $\tau\left(L_{(1,0,1)}\right), \tau\left(L_{(0,1,1)}\right), \tau\left(L_{(1,1,1)}\right)$ (см. пример в $\S 1$ ), а $\tilde{g}: \tilde{Y} \rightarrow \mathbb{P}^{2}$ - накрытие Галуа с группой Галуа $G_{2}=\mathbb{Z} / 2 \mathbb{Z}$, разветвленное вдоль $Q_{i}=g_{1}^{-1}\left(L_{i}\right), i=1,2,3$, и $C_{4}=g_{1}^{-1}\left(\tau\left(L_{(0,0,1)}\right)\right)$, где $Q_{1}, Q_{2}$, $Q_{3}-$ квадрики и $C_{4}-$ квартика в $\mathbb{P}^{2}$ такие, что кривая $B=Q_{1}+Q_{2}+Q_{3}+C_{4}$ имеет шесть особых точек типа $[3,3]$.

TEOPEмA 4.2. Группа автоморфизмов $\operatorname{Aut}(X)$ общей поверхности Кампеделли $X$ изоморфна $(\mathbb{Z} / 2 \mathbb{Z})^{3}$ и совпадает $c$ әруппой $G$ накрывающ, хх преобразований накрытия $f: X \rightarrow \widetilde{\mathbb{P}}^{2}$.

ДокАЗАТЕльство. Пусть $\bar{L} \subset \mathbb{P}^{2}$ - конфигурация Кампеделли, не имеющая тройных точек, и предположим также, что если автоморфизм $\tilde{h}$ плоскости $\mathbb{P}^{2}$ остав- 
ляет неподвижной конфигурацию $\bar{L}$ (т. е. $h(\bar{L})=\bar{L}$ ), то $h=$ id. Рассмотрим накрытие $g: Y \rightarrow \mathbb{P}^{2}$, ассоциированное с эпиморфизмом $\varphi: H_{1}\left(\mathbb{P}^{2} \backslash \bar{L}, \mathbb{Z}\right) \rightarrow G=(\mathbb{Z} / 2 \mathbb{Z})^{3}$, заданным равенствами $\varphi\left(\lambda_{\alpha_{i}}\right)=\alpha_{i}$. Поскольку конфигурация $\bar{L}$ не имеет тройных точек, то $Y=X$ является неособой поверхностью и $g=f$. Морфизм $f$ индуцирует расширение полей $f^{*}\left(\mathbb{C}\left(\mathbb{P}^{2}\right)\right) \subset \mathbb{C}(X)$. Как и в $\S 1$, выберем бесконечно удаленную прямую $L_{\infty}$, координаты $(x, y)$ в $\mathbb{P}^{2} \backslash L_{\infty}$ и отождествим $\mathbb{C}\left(\mathbb{P}^{2}\right)$ с полем рациональных функций $\mathbb{C}(x, y)$.

Рассмотрим элемент $\alpha \in \operatorname{Tors}_{2}(X)=\operatorname{Tors}(X), \alpha \neq 0$. Линейная система $\left|K_{X}+\alpha\right|$ не пуста, и дивизор $D$ принадлежит $\left|K_{X}+\alpha\right|$ для некоторого $\alpha \in$ $\operatorname{Tors}_{2}\left(X_{s}\right)$ тогда и только тогда, когда $2 D=f^{*}(\tilde{L})$ для некоторого элемента $\tilde{L} \in|L|$, где $L$ - прямая в $\mathbb{P}^{2}$. Действительно, по теореме Римана-Роха линейная система $\left|K_{X}+\alpha\right|$ не пуста, так как

$$
\operatorname{dim} H^{2}\left(X, \mathscr{O}_{X}\left(K_{X}+\alpha\right)\right)=\operatorname{dim} H^{0}\left(X, \mathscr{O}_{X}(\alpha)\right)=0 .
$$

Пусть $D_{\alpha} \in\left|K_{X}+\alpha\right|$. Тогда $2 D_{\alpha} \in\left|2 K_{X}\right|$. Из теоремы Римана - Роха получаем, что $\operatorname{dim} H^{0}\left(X, 2 K_{X}\right)=K_{X}^{2}+1=3$. С другой стороны, из утверждения 2.2 следует, что $2 K_{X}=f^{*}(L)$ и $\operatorname{dim} H^{0}\left(\mathbb{P}^{2}, \mathscr{O}_{\mathbb{P}^{2}}(1)\right)=3$. Следовательно, $\left|2 K_{X}\right|=$ $f^{*}(|L|)$ и $D \in\left|K_{X}+\alpha\right|$ для некоторого $\alpha \in \operatorname{Tors}_{2}(X)$ тогда и только тогда, когда $2 D=f^{*}(\tilde{L})$ для некоторого $\tilde{L} \in|L|$.

Легко видеть, что существуют ровно семь прямых $\tilde{L} \in|L|$, для которых дивизоры $f^{*}(\tilde{L})$ делятся на 2 , а именно $L_{\alpha} \subset \bar{L}, \alpha \in \operatorname{Tors}_{2}(X), \alpha \neq 0$. Таким образом, имеем $\frac{1}{2} f^{*}\left(L_{\alpha}\right)=D_{\alpha} \in\left|K_{X}+\alpha\right|$.

Пусть $h: X \rightarrow X$-некоторый автоморфизм. Тогда он индуцирует изоморфизмы $h^{*}: \operatorname{Tors}(X) \rightarrow \operatorname{Tors}(X)$ и

$$
h^{*}: H^{0}\left(X, \mathscr{O}_{X}\left(K_{X}+\alpha\right)\right) \rightarrow H^{0}\left(X, \mathscr{O}_{X}\left(K_{X}+h^{*}(\alpha)\right)\right)
$$

для каждого $\alpha \in \operatorname{Tors}(X)$. Следовательно, $h^{*}\left(D_{\alpha}\right)=D_{h^{*}(\alpha)}$ для $\alpha \in \operatorname{Tors}_{2}(X)$, $\alpha \neq 0$. Автоморфизм $h$ индуцирует действие $h^{*}$ на группе $\operatorname{Div} X$. Имеем

$$
\begin{aligned}
h^{*}\left(f^{*}\left(L_{\alpha_{1}}-L_{\alpha_{2}}\right)\right) & =h^{*}\left(2 D_{\alpha_{1}}-2 D_{\alpha_{2}}\right)=2 D_{h^{*}\left(\alpha_{1}\right)}-2 D_{h^{*}\left(\alpha_{2}\right)} \\
& =f^{*}\left(L_{h^{*}\left(\alpha_{1}\right)}-L_{h^{*}\left(\alpha_{2}\right)}\right)
\end{aligned}
$$

для всех $\alpha_{1}, \alpha_{2} \in \operatorname{Tors}(X), \alpha_{1} \neq \alpha_{2} \neq 0$. Следовательно,

$$
h^{*}\left(f^{*}\left(\frac{l_{\alpha_{1}}(x, y)}{l_{\alpha_{2}}(x, y)}\right)\right)=c_{\alpha_{1}, \alpha_{2}} f^{*}\left(\frac{l_{h^{*}\left(\alpha_{1}\right)}(x, y)}{l_{h^{*}\left(\alpha_{2}\right)}(x, y)}\right),
$$

где $c_{\alpha_{1}, \alpha_{2}}$ - некоторая константа, так как каждая рациональная функция определяется однозначно с точностью до умножения на константу своим дивизором нулей и полюсов. Из (4.2) следует, что $h^{*}$ индуцирует автоморфизм $\tilde{h}^{*}$ поля $\mathbb{C}(x, y)$ такой, что $f^{*} \circ \tilde{h}^{*}=h^{*} \circ f^{*}$, так как функции $\frac{l_{\alpha_{1}}(x, y)}{l_{\alpha_{2}}(x, y)}$ порождают поле $\mathbb{C}(x, y)$. Более того, автоморфизм $\tilde{h}^{*}$ индуцирует автоморфизм $\tilde{h}$ плоскости $\mathbb{P}^{2}$ такой, что $\tilde{h}(\bar{L})=\bar{L}$. Следовательно, $\tilde{h}=\operatorname{id}$ и $h \in \operatorname{Gal}\left(X / \mathbb{P}^{2}\right)$. 
ТеОРема 4.3 (ср. [8]). Пространство модулей $\mathscr{C}$ поверхностей Кампеделли является унирациональным многообразием, $\operatorname{dim} \mathscr{C}=6$.

ДокАЗАТЕльство. Используя те же аргументы, которые использовались в доказательстве теоремы 4.2, можно показать, что поверхности Кампеделли $X_{1}$ и $X_{2}$, определенные с помощью конфигураций прямых Кампеделли $\bar{L}_{1}$ и $\bar{L}_{2}$, изоморфны тогда и только тогда, когда сушествует линейное преобразование $h$ плоскости $\mathbb{P}^{2}$, переводящее $\bar{L}_{1}$ в $\bar{L}_{2}$.

Осуществив подходящее линейное преобразование плоскости $\mathbb{P}^{2}$ и подходящий автоморфизм группы $(\mathbb{Z} / 2 \mathbb{Z})^{3}$, можно предполагать, что в конфигурации прямых $\bar{L}=\sum L_{\alpha}$ прямые $L_{(1,0,0)}, L_{(0,1,0)}, L_{(1,1,0)}$ и $L_{(1,1,1)}$ заданы соответственно уравнениями $z_{0}=0, z_{1}=0, z_{2}=0$ и $z_{0}+z_{1}+z_{2}=0$. Следовательно, конфигурация прямых $\bar{L}$ определяется точкой из подмножества $V$, всюду плотного в $\left(\check{\mathbb{P}}^{2} \backslash\{\text { четыре точки }\}\right)^{3}$. Очевидно, что для любой точки $v_{0} \in V$ множество $A_{v_{0}} \subset V$, состоящее из точек, для которых соответствующие конфигурации прямых $\bar{L}_{v}, v \in A_{v_{0}}$, могут быть преобразованы в $\bar{L}_{v_{0}}$ с помошью линейного преобразования плоскости $\mathbb{P}^{2}$, является конечным. Следовательно, пространство модулей $\mathscr{C}$ является унирациональным многообразием, $\operatorname{dim} \mathscr{C}=6$ (см. также следствия 4.23 и 4.25 ).

4.2. Поверхности Бурниа. Пусть $\bar{L}_{s}=L_{1}+\cdots+L_{9}$ - конфигурация девяти прямых в $\mathbb{P}^{2}$, изображенная на рис. 2 . Эта конфигурация $\bar{L}_{s}$ имеет три четырехкратные точки $p_{1}, p_{2}, p_{3}$ и $s(s=0, \ldots, 4)$ тройных точек $p_{3+i}, 0<i \leqslant s$. Такие конфигурации прямых будем назьвать конфигурациями Бурниа.

Рассмотрим накрытие $g: Y_{s} \rightarrow \mathbb{P}^{2}$, индуцированное эпиморфизмом $\varphi$ : $H_{1}\left(\mathbb{P}^{2} \backslash \bar{L}_{s}, \mathbb{Z}\right) \rightarrow G=(\mathbb{Z} / 2 \mathbb{Z})^{2}$, заданным формулами

$$
\begin{aligned}
& \varphi\left(\lambda_{1}\right)=\varphi\left(\lambda_{2}\right)=\varphi\left(\lambda_{3}\right)=(1,0), \\
& \varphi\left(\lambda_{4}\right)=\varphi\left(\lambda_{5}\right)=\varphi\left(\lambda_{6}\right)=(0,1), \\
& \varphi\left(\lambda_{7}\right)=\varphi\left(\lambda_{8}\right)=\varphi\left(\lambda_{9}\right)=(1,1) .
\end{aligned}
$$

Поверхность $Y_{s}$ имеет $3+s$ особых точек, лежащих над четырехкратными точками $p_{j}, j=1,2,3$, и тройными точками $p_{3+i}, \quad 1 \leqslant i \leqslant s$. Пусть $\sigma: \widetilde{\mathbb{P}}^{2} \rightarrow \mathbb{P}^{2}-$ композиция раздутий этих точек. Обозначим через $E_{j}=\sigma^{-1}\left(p_{j}\right), 1 \leqslant j \leqslant 3+s$, исключительную кривую, лежащую над точкой $p_{j}$, и рассмотрим индуцированное накрытие Галуа $f: X_{s} \rightarrow \widetilde{\mathbb{P}}^{2}$. Данная поверхность $X_{s}$ называется поверхностью Бурниа. Имеем

$$
\varphi\left(\varepsilon_{1}\right)=(1,1), \quad \varphi\left(\varepsilon_{2}\right)=(1,0), \quad \varphi\left(\varepsilon_{3}\right)=(1,1), \quad \varphi\left(\varepsilon_{3+i}\right)=(0,0), \quad 1 \leqslant i \leqslant s .
$$

Следовательно, $E_{j}$ являются кривыми ветвления накрытия $f$ для $j=1,2,3$, а $E_{3+i}$ не являются кривыми ветвления при $i \geqslant 1$. Таким образом, согласно лемме 1.4 поверхность $X_{s}$ является гладкой поверхностью. Заметим, что число тройных и четырехкратных точек конфигурации $\bar{L}$ меньше восьми и каждые четыре из этих точек не лежат на одной и той же прямой. Следовательно, $\widetilde{\mathbb{P}}^{2}$ является поверхностью дель Пещо, возможно, с (-2)-кривыми.

ПРЕДЛОЖЕНИЕ 4.4. Построенные выше поверхности Бурниа $X_{s}$ являются поверхностями общего типа с $K_{X_{s}}^{2}=(6-s), 0 \leqslant s \leqslant 4, u p_{g}=0$. 


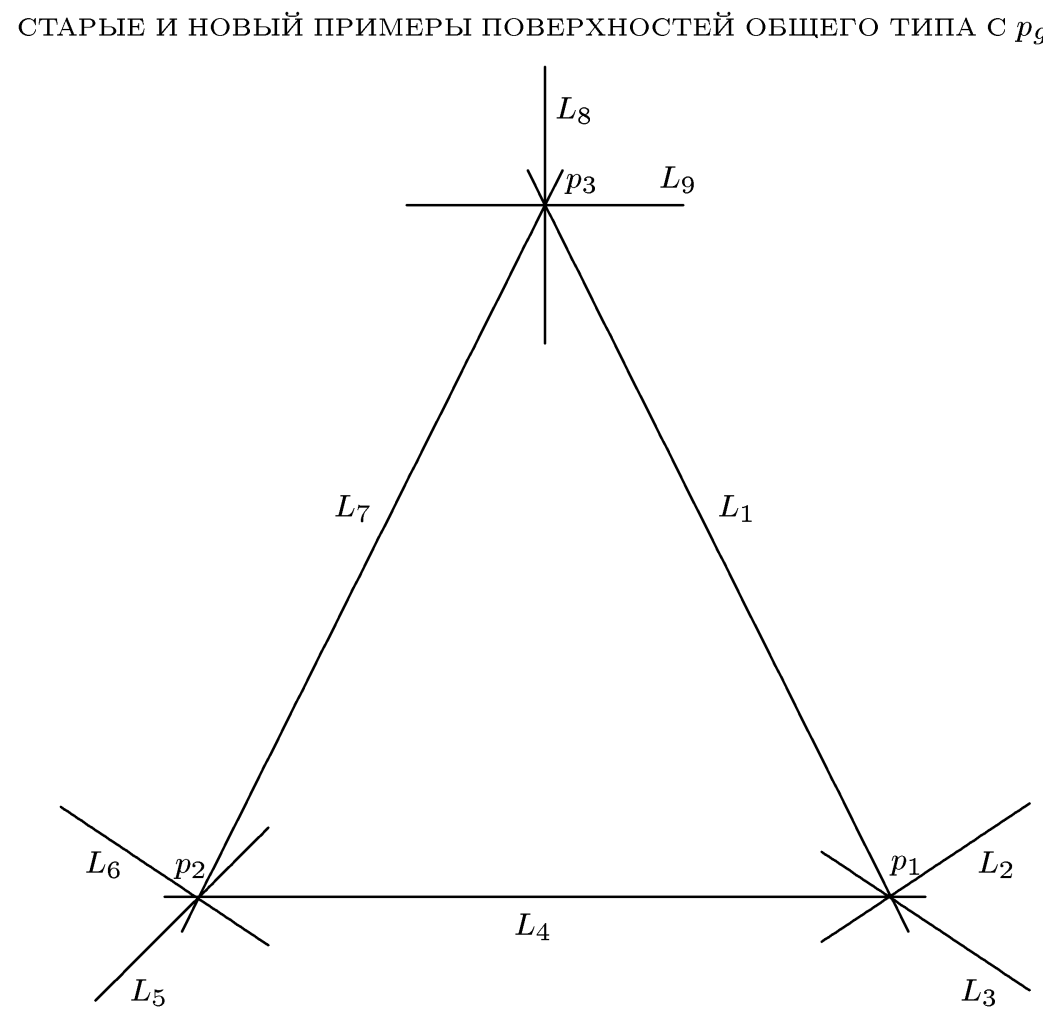

Рис. 2

ДокАЗАТЕЛЬСТво. Согласно утверждению 2.2 имеем

$$
2 K_{X_{s}}=\left|\tilde{f}^{*}\left(3 L-\sum_{i=1}^{3+s} E_{i}\right)\right|,
$$

где $L=\sigma^{*}\left(\mathbb{P}^{1}\right)$ - прообраз прямой $\mathbb{P}^{1} \subset \mathbb{P}^{2}$. Следовательно, $X_{s}$ является поверхностью общего типа и относительно минимальной моделью, так как $\widetilde{\mathbb{P}}^{2}-$ поверхность дель Пеццо, возможно, с (-2)-кривыми. Применив $(2.2)$ и $(2.8)$, легко видеть, что $K_{X_{s}}^{2}=6-s$ и $e(X)=6+s$. Поэтому из формулы Нётера следует, что $p_{a}=1-q+p_{g}=1$. Как и выше, чтобы вычислить $p_{g}$, достаточно вычислить геометрические роды десингуляризаций $\bar{Z}_{i}$ трех циклических накрытий $g_{i}: Z_{i} \rightarrow \mathbb{P}^{2}$, соответствуюших трем эпиморфизмам групш $G=(\mathbb{Z} / 2 \mathbb{Z})^{2}$ в циклическую группу $\mathbb{Z} / 2 \mathbb{Z}$ :

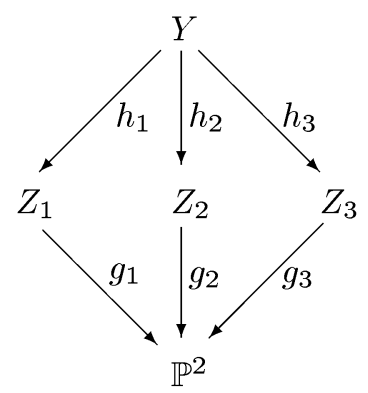


в которой $g=g_{i} \circ h_{i}$ для $i=1,2,3$. Эти накрытия задаются в неоднородных координатах соответственно уравнениями

$$
\begin{aligned}
& w_{1}^{2}=l_{1} l_{2} l_{3} l_{4} l_{5} l_{6}, \\
& w_{2}^{2}=l_{4} l_{5} l_{6} l_{7} l_{8} l_{9}, \\
& w_{3}^{2}=l_{1} l_{2} l_{3} l_{7} l_{8} l_{9} .
\end{aligned}
$$

Применив утверждение 3.2 , можно легко показать, что геометрический род каждого из этих накрытий обращается в нуль, так как каждая из конфигураций, заданных соответственно уравнениями $l_{1} l_{2} l_{3} l_{4} l_{5} l_{6}=0, l_{4} l_{5} l_{6} l_{7} l_{8} l_{9}=0$ и $l_{1} l_{2} l_{3} l_{7} l_{8} l_{9}=0$, имеет четырехкратную точку. Таким образом, $X_{s}$ имеет геометрический род $p_{g}=0$.

Обозначим через $\tilde{L}_{j}$ собственный прообраз $\sigma^{-1}\left(L_{j}\right)$ прямой $L_{j} \subset \bar{L}_{s}$. Тогда дивизор $\sum \tilde{L}_{j}+\sum_{i=1}^{3} E_{i}$ является дивизором ветвления накрытия $f$. Положим

$$
\begin{aligned}
2 C_{j} & =f^{*}\left(\tilde{L}_{j}\right), & & j=1, \ldots, 9, \\
2 D_{i} & =f^{*}\left(E_{i}\right), & & i=1,2,3, \\
D_{k} & =f^{*}\left(E_{k}\right), & & 3<k \leqslant 3+s,
\end{aligned}
$$

и обозначим через $t\left(L_{j}\right)$ число особых точек конфигурации $\bar{L}_{s}$, лежащих на прямой $L_{j}$.

УТВЕРЖДЕНИЕ 4.5. Имеем:

(i) кривые $C_{j}, \quad j=1, \ldots, 9, u D_{i}, \quad i=1, \ldots, 3+s$, являются неособыми;

(ii) геометрический род кривой $C_{j}, j=1, \ldots, 9$, равен $g\left(D_{i}\right)=3-t\left(L_{j}\right)$;

(iii) геометрический род кривой $D_{i}$ равен $g\left(D_{i}\right)=1$, если $i=1,2,3$, u $g\left(D_{i}\right)=0$, если $3<i \leqslant 3+s$;

(iv) индекс самопересечения кривой $C_{j}$ при $j=1, \ldots, 9$ равен $\left(C_{j}^{2}\right)_{X_{s}}=1$ $t\left(L_{j}\right)$

(v) индекс самопересечения кривой $D_{i}$ равен $\left(D_{i}^{2}\right)_{X_{s}}=-1$, если $i=1,2,3$, $u\left(D_{i}^{2}\right)_{X_{s}}=-4$, если $3<i \leqslant 3+s$.

ДокаЗАТЕЛЬСтво. Утверждение (i) очевидно.

Докажем утверждения (ii), (iii). Ограничение накрывающего морфизма $f$ на кривую $C_{j}, j=1, \ldots, 9$, является двулистным накрытием рациональной кривой, разветвленным в $8-2 t\left(L_{j}\right)$ точках. Следовательно, $g\left(C_{j}\right)=3-t\left(L_{j}\right)$.

Ограничение морфизма $f$ на кривую $D_{i}, i=1,2,3$, является двулистным накрытием, разветвленным в четырех точках. Следовательно, $g\left(D_{i}\right)=1$. Аналогично, ограничение $f$ на $D_{i}, 3<i \leqslant 3+s$, является бидвойным накрытием рациональной кривой, разветвленным в трех точках. Следовательно, геометрический род $g\left(D_{i}\right)=0$.

Теперь докажем утверждения (iv), (v). Так как $\left(f^{*}(D), f^{*}(D)\right)_{X_{s}}=\operatorname{deg} f \times$ $(D, D)_{\widetilde{\mathbb{P}}^{2}}=4(D, D)_{\widetilde{\mathbb{P}}^{2}}$ для любого дивизора $D$ на $\widetilde{\mathbb{P}}^{2}$, то утверждение следует из равенств: $\left(\tilde{L}_{j}^{2}\right)_{\widetilde{\mathbb{P}} 2}=1-t\left(L_{j}\right)$ для $j=1, \ldots, 9$ и $\left(\widetilde{E}_{i}^{2}\right)_{\widetilde{\mathbb{P}} 2}=-1$ для $i=1, \ldots, 3+s$.

Рассмотрим универсальное накрытие Галуа $\bar{f}_{s}: \bar{X}_{s} \rightarrow \widetilde{\mathbb{P}}^{2}$ и универсальное неразветвленное накрытие $h_{s, \varphi}: \bar{X}_{s} \rightarrow X_{s}$ относительно эпиморфизма $\varphi:$ 
$H_{1}\left(\mathbb{P}^{2} \backslash \bar{L}_{s}, \mathbb{Z}\right) \rightarrow G=(\mathbb{Z} / 2 \mathbb{Z})^{2}$ такие, что $\bar{f}_{s}=f_{s} \circ h_{s, \varphi}$. Напомним, что накрытие $\bar{f}_{s}$ индуцировано эпиморфизмом $\psi_{s, \varphi}: H_{1}\left(\mathbb{P}^{2} \backslash \bar{L}_{s}, \mathbb{Z}\right) \rightarrow(\mathbb{Z} / q \mathbb{Z})^{k_{s, \varphi}}$, где $(\mathbb{Z} / q \mathbb{Z})^{k_{s, \varphi}}$ и $\psi_{s, \varphi}$ определены уравнениями

$$
\begin{gathered}
\sum_{j=1}^{9} x_{j}=0 \\
x_{j_{1}(i)}+x_{j_{2}(i)}+x_{j_{3}(i)}=0, \quad 3<i \leqslant 3+s,
\end{gathered}
$$

где для каждого $i$ тройка $\left(j_{1}(i), j_{2}(i), j_{3}(i)\right)$ является множеством индексов прямых $L_{j}$ таких, что $p_{i}=L_{j_{1}(i)} \cap L_{j_{2}(i)} \cap L_{j_{3}(i)}$.

В случае $s=0$ (нет тройных точек) имеем $k_{0, \varphi}=8$ и

$$
\operatorname{deg} h_{0, \varphi}=2^{6} .
$$

В случае $s=1$ будем предполагать, что $p_{4}=L_{3} \cap L_{6} \cap L_{9}$, тем самьм получаем, что группа $(\mathbb{Z} / q \mathbb{Z})^{k_{1, \varphi}}$ и эпиморфизм $\psi_{1, \varphi}$ определяются уравнениями

$$
\begin{gathered}
\sum_{j=1}^{9} x_{j}=0, \\
x_{3}+x_{6}+x_{9}=0 .
\end{gathered}
$$

Следовательно, $k_{1, \varphi}=7$ и

$$
\operatorname{deg} h_{1, \varphi}=2^{5} .
$$

В случае $s=2$ будем предполагать, что $p_{4}=L_{3} \cap L_{6} \cap L_{9}$, а $p_{5}$ (см. рис. 3 и 4) является пересечением либо прямых $L_{2} \cap L_{5} \cap L_{8}$ (конфигурация $\bar{L}_{2}^{\prime}$ ), либо $L_{2} \cap L_{5} \cap L_{9}$ (конфигурация $\bar{L}_{2}^{\prime \prime}$ ).

В случае конфигурации $\bar{L}_{2}^{\prime}$ получаем, что группа $(\mathbb{Z} / q \mathbb{Z})^{k_{2, \varphi}}$ и эпиморфизм $\psi_{2, \varphi}$ определяются уравнениями

$$
\begin{gathered}
\sum_{j=1}^{9} x_{j}=0, \\
x_{3}+x_{6}+x_{9}=0, \\
x_{2}+x_{5}+x_{8}=0,
\end{gathered}
$$

а в случае конфигурации $\bar{L}_{2}^{\prime \prime}$ группа и эпиморфизм определяются уравнениями

$$
\begin{gathered}
\sum_{j=1}^{9} x_{j}=0, \\
x_{3}+x_{6}+x_{9}=0, \\
x_{2}+x_{5}+x_{9}=0 .
\end{gathered}
$$




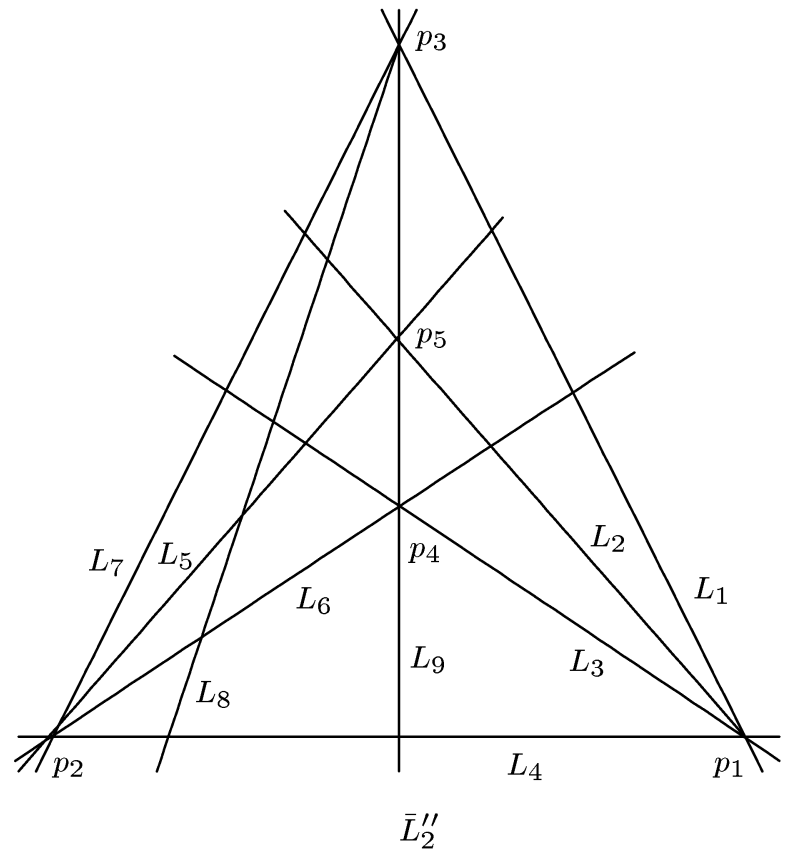

Рис. 3

Следовательно, в обоих случаях получаем, что $k_{2, \varphi}=6$ и

$$
\operatorname{deg} h_{2, \varphi}=2^{4}
$$

В случае $s=3$ будем предполагать, что $p_{4}=L_{3} \cap L_{6} \cap L_{9}, p_{5}=L_{2} \cap$ $L_{5} \cap L_{9}$ и $p_{6}=L_{2} \cap L_{6} \cap L_{8}$. Следовательно, группа $(\mathbb{Z} / q \mathbb{Z})^{k_{3, \varphi}}$ и эпиморфизм $\psi_{3, \varphi}$ определяются уравнениями

$$
\begin{gathered}
\sum_{j=1}^{9} x_{j}=0, \\
x_{3}+x_{6}+x_{9}=0, \\
x_{2}+x_{5}+x_{9}=0, \\
x_{2}+x_{6}+x_{8}=0 .
\end{gathered}
$$

Поэтому $k_{3, \varphi}=5$ и

$$
\operatorname{deg} h_{1, \varphi}=2^{3} \text {. }
$$

В случае $s=4$ будем предполагать, что $p_{4}=L_{3} \cap L_{6} \cap L_{9}, p_{5}=L_{2} \cap L_{5} \cap L_{9}$, $p_{6}=L_{2} \cap L_{6} \cap L_{8}$ и $p_{7}=L_{3} \cap L_{5} \cap L_{8}$. Эта конфигурация прямых $\bar{L}_{4}$ изображена на рис. 5. 


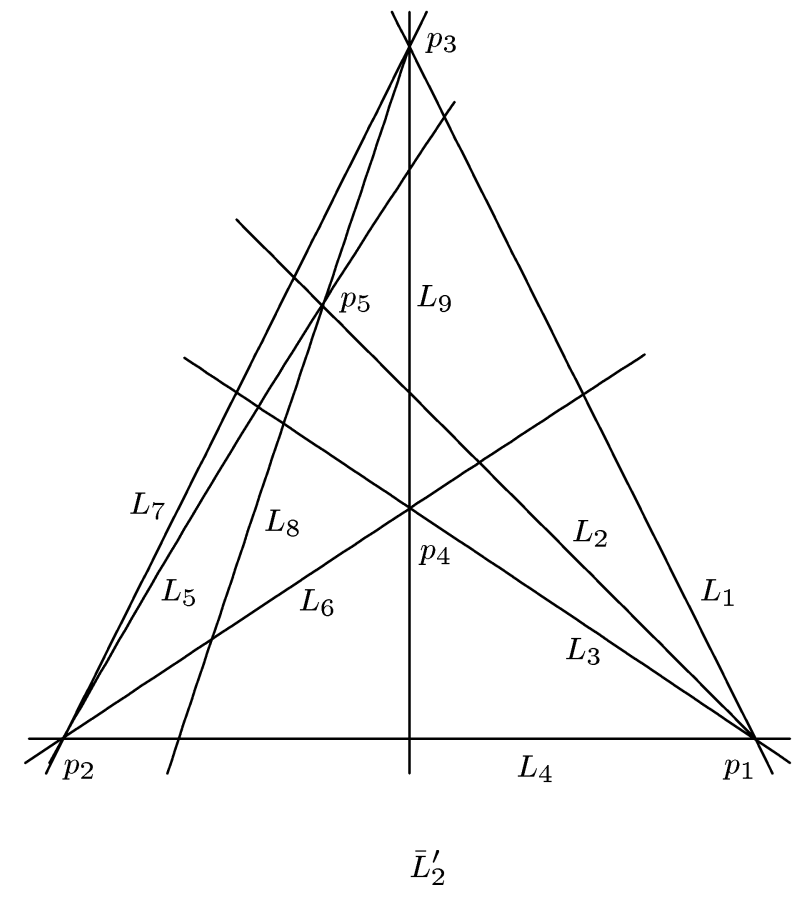

Рис. 4

Следовательно, группа $(\mathbb{Z} / q \mathbb{Z})^{k_{4, \varphi}}$ и эпиморфизм $\psi_{4, \varphi}$ определяются уравнениями

$$
\begin{gathered}
\sum_{j=1}^{9} x_{j}=0, \\
x_{3}+x_{6}+x_{9}=0, \\
x_{2}+x_{5}+x_{9}=0, \\
x_{2}+x_{6}+x_{8}=0, \\
x_{3}+x_{5}+x_{8}=0 .
\end{gathered}
$$

Легко видеть, что ранг линейной системы (4.14) над $\mathbb{Z} / 2 \mathbb{Z}$ равен четырем. Следовательно, $k_{4, \varphi}=5$ и

$$
\operatorname{deg} h_{4, \varphi}=2^{3} .
$$

УТВЕРЖДЕНИЕ 4.6. Пусть $X_{s}$ является поверхностью Бурниа, а $\alpha \in$ $\operatorname{Tors}_{2}\left(X_{s}\right)=\operatorname{Tors}_{2} H^{2}\left(X_{s}, \mathbb{Z}\right), \alpha \neq 0$. Тогда линейная система $\left|K_{X_{s}}+\alpha\right|$ не пуста и дивизор $D$ принадлежит $\left|K_{X_{s}}+\alpha\right|$ для некоторого $\alpha \in \operatorname{Tors}_{2}\left(X_{s}\right), \alpha \neq 0$, тогда и только тогда, когда $2 D=f^{*}(\bar{D})$ для некоторого $\bar{D} \in\left|3 L-\sum_{i=1}^{3+s} E_{i}\right|$.

ДокАзАтеЛЬСтво. По теореме Римана-Роха линейная система $\left|K_{X_{s}}+\alpha\right|$ не пуста, так как

$$
\operatorname{dim} H^{2}\left(X_{s}, \mathscr{O}_{X_{s}}\left(K_{X_{s}}+\alpha\right)\right)=\operatorname{dim} H^{0}\left(X_{s}, \mathscr{O}_{X_{s}}(\alpha)\right)=0 .
$$




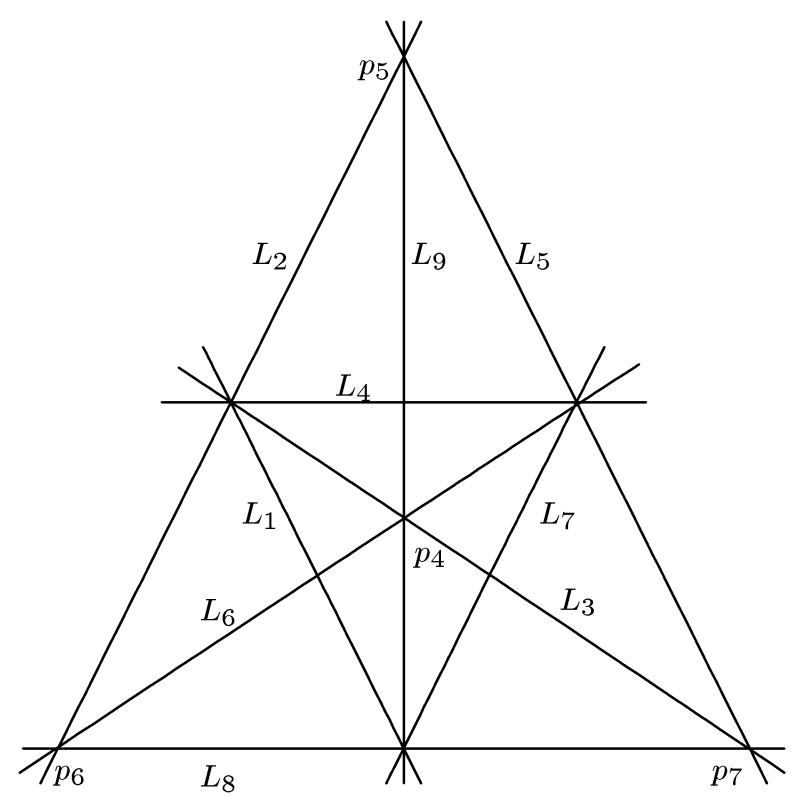

Рис. 5

Пусть $D_{\alpha} \in\left|K_{X_{s}}+\alpha\right|$. Тогда $2 D_{\alpha} \in\left|2 K_{X_{s}}\right|$. Из теоремы Римана-Роха следует, что

$$
\operatorname{dim} H^{0}\left(X_{s}, 2 K_{X_{s}}\right)=K_{X_{s}}^{2}+1=7-s .
$$

С другой стороны, согласно утверждению 2.2

$$
\begin{gathered}
2 K_{X_{s}}=f^{*}\left(3 L-\sum_{i=1}^{3+s} E_{i}\right) \\
\operatorname{dim} H^{0}\left(\widetilde{\mathbb{P}}^{2}, \mathscr{O}_{\widetilde{\mathbb{P}}^{2}}\left(3 L-\sum_{i=1}^{3+s} E_{i}\right)\right)=7-s
\end{gathered}
$$

Следовательно,

$$
\left|2 K_{X_{s}}\right|=f^{*}\left(\left|3 L-\sum_{i=1}^{3+s} E_{i}\right|\right)
$$

и $D \in\left|K_{X_{s}}+\alpha\right|$ для некоторого $\alpha \in \operatorname{Tors}_{2}\left(X_{s}\right)$ тогда и только тогда, когда $2 D=f^{*}(\bar{D})$ для некоторого дивизора $\bar{D} \in\left|3 L-\sum_{i=1}^{3+s} E_{i}\right|$.

ПРЕДЛОЖЕНИЕ 4.7. Группа 2-кручения поверхности Бурниа $X_{s}$ изоморфна $\operatorname{Tors}_{2}\left(X_{s}\right) \simeq(\mathbb{Z} / 2 \mathbb{Z})^{6-s}$ nрu $s \leqslant 3 u \operatorname{Tors}_{2}\left(X_{4}\right) \simeq(\mathbb{Z} / 2 \mathbb{Z})^{3}$. 


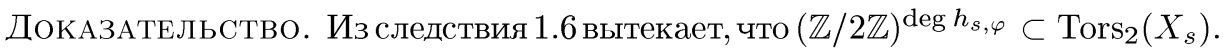
Заметим, что $\operatorname{deg} h_{s, \varphi}=6-s$, если $s \leqslant 3$, и $\operatorname{deg} h_{4, \varphi}=3$. Из утверждения 4.6 следует, что чтобы доказать предложение в каждом из случаев $s=4,3,2,1,0$, достаточно показать, что существует ровно $2^{\operatorname{deg} h_{s, \varphi}}-1$ полных непрерывных систем дивизоров $\bar{D}$, принадлежаших линейной системе $\left|3 L-\sum_{i=1}^{3+s} E_{i}\right|$ и таких, что прообраз $f^{*}(\bar{D})$ каждого дивизора $\bar{D}$ делится на два (т. е. $\left.f^{*}(\bar{D})=2 D\right)$, и любые два дивизора $\frac{1}{2} f^{*}\left(\bar{D}_{i}\right), \frac{1}{2} f^{*}\left(\bar{D}_{j}\right)$, принадлежашие разным системам, не являются линейно эквивалентньми.

Можно проверить, что:

a) в случае $s=4$ такими элементами $\bar{D} \in\left|3 L-\sum_{i=1}^{7} E_{i}\right|$, для которых дивизор $f^{*}(\bar{D})$ делится на два, являются

$$
\begin{array}{lll}
\tilde{L}_{3}+\tilde{L}_{6}+\tilde{L}_{9}+2 E_{4}, & \tilde{L}_{2}+\tilde{L}_{5}+\tilde{L}_{9}+2 E_{5}, & \tilde{L}_{2}+\tilde{L}_{6}+\tilde{L}_{8}+2 E_{6}, \\
\tilde{L}_{3}+\tilde{L}_{5}+\tilde{L}_{8}+2 E_{7}, & \tilde{L}_{2}+\tilde{L}_{3}+\tilde{L}_{7}+E_{1}, & \tilde{L}_{5}+\tilde{L}_{6}+\tilde{L}_{1}+E_{2}, \\
\tilde{L}_{8}+\tilde{L}_{9}+\tilde{L}_{4}+E_{3} ; &
\end{array}
$$

б) в случае $s=3$ элементами $\bar{D} \in\left|3 L-\sum_{i=1}^{6} E_{i}\right|$, для которых дивизор $f^{*}(\bar{D})$ делится на два, являются

$$
\begin{array}{lll}
\tilde{L}_{3}+\tilde{L}_{6}+\tilde{L}_{9}+2 E_{4}, & \tilde{L}_{2}+\tilde{L}_{5}+\tilde{L}_{9}+2 E_{5}, & \tilde{L}_{2}+\tilde{L}_{6}+\tilde{L}_{8}+2 E_{6}, \\
\tilde{L}_{3}+\tilde{L}_{5}+\tilde{L}_{8}, & \tilde{L}_{2}+\tilde{L}_{3}+\tilde{L}_{7}+E_{1}, & \tilde{L}_{5}+\tilde{L}_{6}+\tilde{L}_{1}+E_{2}, \\
\tilde{L}_{8}+\tilde{L}_{9}+\tilde{L}_{4}+E_{3} ; & &
\end{array}
$$

в) в случае $s=2$ и $\bar{L}_{2}=\bar{L}_{2}^{\prime}$ элементами $\bar{D} \in\left|3 L-\sum_{i=1}^{5} E_{i}\right|$, для которых $f^{*}(\bar{D})$ делится на два, являются

$$
\begin{array}{lll}
\tilde{L}_{2}+\tilde{L}_{3}+\tilde{L}_{7}+E_{1}, & \tilde{L}_{1}+\tilde{L}_{5}+\tilde{L}_{6}+E_{2}, & \tilde{L}_{4}+\tilde{L}_{8}+\tilde{L}_{9}+E_{3}, \\
\tilde{L}_{1}+\tilde{L}_{2}+\tilde{L}_{6}+E_{1}, & \tilde{L}_{2}+\tilde{L}_{6}+\tilde{L}_{7}+E_{2}, & \tilde{L}_{1}+\tilde{L}_{3}+\tilde{L}_{5}+E_{1}, \\
\tilde{L}_{3}+\tilde{L}_{5}+\tilde{L}_{7}+E_{2}, & \tilde{L}_{2}+\tilde{L}_{4}+\tilde{L}_{9}+E_{1}, & \tilde{L}_{2}+\tilde{L}_{7}+\tilde{L}_{9}+E_{3}, \\
\tilde{L}_{3}+\tilde{L}_{4}+\tilde{L}_{8}+E_{1}, & \tilde{L}_{3}+\tilde{L}_{7}+\tilde{L}_{8}+E_{3}, & \tilde{L}_{1}+\tilde{L}_{5}+\tilde{L}_{9}+E_{3}, \\
\tilde{L}_{4}+\tilde{L}_{5}+\tilde{L}_{9}+E_{2}, & \tilde{L}_{1}+\tilde{L}_{6}+\tilde{L}_{8}+E_{3}, & \tilde{L}_{4}+\tilde{L}_{6}+\tilde{L}_{8}+E_{2} ;
\end{array}
$$

г) в случае $s=2$ и $\bar{L}_{2}=\bar{L}_{2}^{\prime \prime}$ элементами $\bar{D} \in\left|3 L-\sum_{i=1}^{5} E_{i}\right|$, для которых $f^{*}(\bar{D})$ делится на два, являются

$$
\begin{array}{lll}
\tilde{L}_{2}+\tilde{L}_{3}+\tilde{L}_{7}+E_{1}, & \tilde{L}_{1}+\tilde{L}_{5}+\tilde{L}_{6}+E_{2}, & \tilde{L}_{4}+\tilde{L}_{8}+\tilde{L}_{9}+E_{3}, \\
\tilde{L}_{1}+\tilde{L}_{2}+\tilde{L}_{6}+E_{1}, & \tilde{L}_{2}+\tilde{L}_{6}+\tilde{L}_{7}+E_{2}, & \tilde{L}_{1}+\tilde{L}_{3}+\tilde{L}_{5}+E_{1}, \\
\tilde{L}_{3}+\tilde{L}_{5}+\tilde{L}_{7}+E_{2}, & \tilde{L}_{1}+\tilde{L}_{4}+\tilde{L}_{9}+E_{1}, & \tilde{L}_{4}+\tilde{L}_{7}+\tilde{L}_{9}+E_{2}, \\
\tilde{L}_{2}+\tilde{L}_{5}+\tilde{L}_{9}+2 E_{5}, & \tilde{L}_{3}+\tilde{L}_{6}+\tilde{L}_{9}+2 E_{4}, & \tilde{L}_{2}+\tilde{L}_{6}+\tilde{L}_{8} \\
\tilde{L}_{1}+\tilde{L}_{7}+\tilde{L}_{9}+2 E_{3}, & 2 \tilde{L}_{4}+\tilde{L}_{9}+E_{1}+E_{2}, & \tilde{L}_{3}+\tilde{L}_{5}+\tilde{L}_{8} .
\end{array}
$$

Можно проверить, что в случае $s=1$ существует ровно 31 дивизор $\bar{D} \in \mid 3 L-$ $\sum_{i=1}^{4} E_{i} \mid$, для которых $f^{*}(\bar{D})$ делится на два, а в случае $s=0$ сушествует ровно 63 
полных непрерьвных систем дивизоров $\bar{D}$ в $\left|3 L-\sum_{i=1}^{3} E_{i}\right|$, для которых $f^{*}(\bar{D})$ делится на два. Заметим только, что в случае $s=0$ среди этих систем дивизоров 60 систем состоят из одного дивизора, а последние 3 образуют одномерные линейные системы, а именно

$$
\tilde{L}_{1}+2 \tilde{L}_{p_{2}}+E_{2}, \quad \tilde{L}_{4}+2 \tilde{L}_{p_{3}}+E_{3}, \quad \tilde{L}_{7}+2 \tilde{L}_{p_{1}}+E_{1},
$$

где $\tilde{L}_{p_{i}}=\sigma^{-1}\left(L_{p_{i}}\right)$ - собственный прообраз прямой, принадлежащей пучку прямых, проходящих через точку $p_{i}$. Эти три пучка соответствуют трем элементам, скажем $\alpha_{1}, \alpha_{2}, \alpha_{3} \in \operatorname{Tors}_{2}\left(X_{0}\right)$, для которых $\operatorname{dim} H^{1}\left(X_{0}, \mathscr{O}_{X_{0}}\left(\alpha_{i}\right)\right)=1$, и эти три элемента "приходят" из трех иррегулярных промежуточных циклических накрытий универсального накрытия Галуа $\bar{f}_{0}: \bar{X}_{0} \rightarrow \widetilde{\mathbb{P}}^{2}$ относительно эпиморфизма $\varphi: H_{1}\left(\mathbb{P}^{2} \backslash \bar{L}_{0}, \mathbb{Z}\right) \rightarrow G=(\mathbb{Z} / 2 \mathbb{Z})^{2}$ (см. далее конец доказательства утверждения 4.8).

УТВЕРЖДЕНИЕ 4.8. При $s \geqslant 1$ поверхности $\bar{X}_{s}$ являются регулярныцми, m.е. иррегулярности $q\left(\bar{X}_{s}\right)=0$ и $q\left(\bar{X}_{0}\right)=3$.

ДокАЗАТЕЛЬСтво. Арифметический род $p_{a}$ поверхности равен $p_{a}=p_{g}-q+1$. Следовательно, чтобы вычислить $q$, достаточно вычислить $p_{a}$ и $p_{g}$.

Имеем $p_{a}\left(X_{s}\right)=1$. Следовательно, арифметический род

$$
p_{a}\left(\bar{X}_{s}\right)=2^{k_{s, \varphi}-2},
$$

так как $h_{s}$ является неразветвленным накрытием и $\operatorname{deg} h_{s}=2^{k_{s, \varphi}-2}$.

Вычислим геометрический род $p_{g}\left(\bar{X}_{s}\right)$ в случае $s \geqslant 2$, а вычисление геометрического рода в оставшихся случаях будет оставлено читателю, так как эти вычисления используют одни и те же идеи. Как и в $\S 3$, чтобы вычислить $p_{g}$, достаточно вычислить геометрические роды $2^{k_{s, \varphi}}-1$ циклических накрытий, соответствующих $2^{k_{s, \varphi}}-1$ эпиморфизмам $\psi_{m}, m=1, \ldots, 2^{k_{s, \varphi}}-1$, из группы $G_{u, \varphi}=(\mathbb{Z} / 2 \mathbb{Z})^{k_{s, \varphi}}$ в циклическую группу $\mathbb{Z} / 2 \mathbb{Z}$, где группа $G_{u, \varphi}$ изоморфна подгруппе группы $(\mathbb{Z} / 2 \mathbb{Z})^{9}$, заданной в координатах $\left(x_{1}, \ldots, x_{9}\right)$ одной из линейных систем уравнений (4.5)-(4.14).

Легко видеть, что сушествует взаимно однозначное соответствие $\gamma$ между эпиморфизмами $\psi_{m}$ и элементами $\left(x_{1}, \ldots, x_{9}\right) \in G_{u, \varphi}$ такими, что для $\gamma\left(\psi_{m}\right)=$ $\left(x_{1}, \ldots, x_{9}\right)$ циклическое накрытие, соответствуюшее $\psi_{m}$, задается уравнением

$$
w_{m}^{2}=\prod_{x_{i}=1} l_{i}
$$

Из утверждения 3.2 следует, что вклад в геометрический род поверхности $\bar{X}_{s}$ может быть сделан только циклическими накрытиями, соответствующими эпиморфизмам $\psi_{m}$, для которых сумма координат вектора $\gamma\left(\psi_{m}\right)$ не меньше шести, и если она равна шести, то соответствующий дивизор ветвления не должен иметь четырехкратных точек.

В случаях $s=3$ и $s=4$ (см. линейные системы уравнений (4.12) и (4.14)) имеем ровно семь таких накрытий, заданных уравнениями

$$
\begin{aligned}
& z_{1}^{2}=l_{1} l_{2} l_{3} l_{5} l_{6} l_{7}, \quad z_{2}^{2}=l_{1} l_{4} l_{5} l_{6} l_{8} l_{9}, \quad z_{3}^{2}=l_{2} l_{3} l_{4} l_{7} l_{8} l_{9}, \\
& z_{4}^{2}=l_{1} l_{2} l_{4} l_{5} l_{7} l_{8}, \quad z_{5}^{2}=l_{1} l_{3} l_{4} l_{5} l_{7} l_{9}, \quad z_{6}^{2}=l_{1} l_{3} l_{4} l_{6} l_{7} l_{8}, \\
& z_{7}^{2}=l_{1} l_{2} l_{4} l_{6} l_{7} l_{9} .
\end{aligned}
$$


Следовательно, $p_{g}\left(\bar{X}_{3}\right)=p_{g}\left(\bar{X}_{4}\right)=7$ и $q\left(\bar{X}_{3}\right)=q\left(\bar{X}_{4}\right)=0$.

В случае, когда $s=2$ и конфигурация $\bar{L}_{2}=\bar{L}_{2}^{\prime}$ (см. линейную систему уравнений (4.9)), имеем ровно 15 таких накрытий, заданных уравнениями

$$
\begin{aligned}
z_{1}^{2} & =l_{1} l_{2} l_{3} l_{5} l_{6} l_{7}, \quad z_{2}^{2}=l_{1} l_{2} l_{3} l_{7} l_{8} l_{9}, \quad z_{3}^{2}=l_{1} l_{4} l_{5} l_{6} l_{8} l_{9}, \\
z_{4}^{2} & =l_{1} l_{2} l_{4} l_{6} l_{8} l_{9}, \quad z_{5}^{2}=l_{1} l_{3} l_{4} l_{5} l_{8} l_{9}, \quad z_{6}^{2}=l_{1} l_{2} l_{4} l_{5} l_{6} l_{9}, \\
z_{7}^{2} & =l_{1} l_{3} l_{4} l_{5} l_{6} l_{8}, \quad z_{8}^{2}=l_{1} l_{2} l_{3} l_{6} l_{7} l_{8}, \quad z_{9}^{2}=l_{1} l_{2} l_{3} l_{5} l_{7} l_{9}, \\
z_{10}^{2} & =l_{2} l_{3} l_{4} l_{5} l_{7} l_{9}, \quad z_{11}^{2}=l_{2} l_{3} l_{4} l_{6} l_{7} l_{8}, \quad z_{12}^{2}=l_{2} l_{4} l_{5} l_{6} l_{7} l_{9}, \\
z_{13}^{2} & =l_{3} l_{4} l_{5} l_{6} l_{7} l_{8}, \quad z_{14}^{2}=l_{2} l_{4} l_{6} l_{7} l_{8} l_{9}, \quad z_{15}^{2}=l_{3} l_{4} l_{5} l_{7} l_{8} l_{9} .
\end{aligned}
$$

Следовательно, $p_{g}\left(\bar{X}_{2}^{\prime}\right)=15$ и $q\left(\bar{X}_{2}^{\prime}\right)=0$.

В случае, когда $s=2$ и конфигурация $\bar{L}_{2}=\bar{L}_{2}^{\prime \prime}$ (см. линейную систему уравнений (4.10)), имеем также ровно 15 таких накрытий, заданных уравнениями

$$
\begin{aligned}
& z_{1}^{2}=l_{1} l_{2} l_{3} l_{5} l_{6} l_{7}, \quad z_{2}^{2}=l_{1} l_{4} l_{5} l_{6} l_{8} l_{9}, \quad z_{3}^{2}=l_{2} l_{3} l_{4} l_{7} l_{8} l_{9}, \\
& z_{4}^{2}=l_{1} l_{2} l_{4} l_{5} l_{7} l_{8}, \quad z_{5}^{2}=l_{1} l_{3} l_{4} l_{5} l_{7} l_{9}, \quad z_{6}^{2}=l_{1} l_{3} l_{4} l_{6} l_{7} l_{8}, \\
& z_{7}^{2}=l_{1} l_{2} l_{4} l_{6} l_{7} l_{9}, \quad z_{8}^{2}=l_{1} l_{3} l_{4} l_{5} l_{7} l_{9}, \quad z_{9}^{2}=l_{1} l_{2} l_{4} l_{6} l_{8} l_{9}, \\
& z_{10}^{2}=l_{1} l_{3} l_{4} l_{5} l_{8} l_{9}, \quad z_{11}^{2}=l_{1} l_{2} l_{3} l_{7} l_{8} l_{9}, \quad z_{12}^{2}=l_{2} l_{3} l_{4} l_{7} l_{8} l_{9}, \\
& z_{13}^{2}=l_{2} l_{4} l_{6} l_{7} l_{8} l_{9}, \quad z_{14}^{2}=l_{3} l_{4} l_{5} l_{7} l_{8} l_{9}, \quad z_{15}^{2}=l_{1} l_{2} l_{3} l_{4} l_{5} l_{6} l_{7} l_{8} .
\end{aligned}
$$

Кривая ветвления 15-го двойного накрытия имеет степень 8 и две четверных точки. Следовательно, геометрический род этого накрытия равен единище. Таким образом, снова имеем $p_{g}\left(\bar{X}_{2}^{\prime \prime}\right)=15$ и $q\left(\bar{X}_{2}^{\prime \prime}\right)=0$.

Оставшиеся два случая оставляем для проверки читателю, заметим только, что ненулевой вклад в иррегулярность поверхности $\bar{X}_{0}$ дают только следующие циклические накрытия:

$$
z_{1}^{2}=l_{1} l_{2} l_{3} l_{4}, \quad z_{2}^{2}=l_{1} l_{7} l_{8} l_{9}, \quad z_{3}^{2}=l_{4} l_{5} l_{6} l_{7} .
$$

СлЕДСТВИЕ 4.9. Фундаментальная группа поверхности Бурниа $X_{0}$ является неабелевой бесконечной группой.

ДоКАЗАТЕЛЬСТво следует из утверждения 4.8 .

Легко видеть, что с точностью до линейных преобразований на $\mathbb{P}^{2}$ сушествует только одна конфигурация Бурниа $\bar{L}_{4}$ (изображенная на рис. 5 ).

Зафиксируем однородные координаты $\left(z_{0}: z_{1}: z_{2}\right)$ в $\mathbb{P}^{2}$. Обозначим через $\mathscr{B}_{6-s}=\left\{\bar{L}_{s}=L_{1}+\cdots+L_{9}\right\}, s \leqslant 4$, семейство упорядоченных конфигураций Бурниа таких, что $L_{1}, L_{4}$ и $L_{7}$ заданы соответственно уравнениями $z_{0}=0$, $z_{1}=0$ и $z_{2}=0$, а $p_{4}=(1: 1: 1)$ (в случае $s=0$ точка $p_{4}-$ это пересечение $\left.L_{3} \cap L_{9}\right)$. Легко видеть, что любая конфигурация Бурниа может быть преобразована в конфигурацию, принадлежащую $\mathscr{B}_{6-s}$ с помощью линейного преобразования плоскости $\mathbb{P}^{2}$. Обозначим через $F_{s}: \mathscr{X}_{6-s} \rightarrow \mathscr{B}_{6-s}$ семейство поверхностей Бурниа со слоем $F_{s}^{-1}\left(\bar{L}_{s}\right)=X_{s}$ над точкой $\bar{L}_{s} \in \mathscr{B}_{6-s}$, где $X_{s}$ - поверхность Бурниа, определенная с помошью конфигурации прямых $\bar{L}_{s}$. 
Если $s=3$, то конфигурация $\bar{L}_{3} \in \mathscr{B}_{3}$ однозначно определяется точкой $p_{5}=$ $L_{2} \cap L_{5} \cap L_{9} \in L_{9}$, так как прямые $L_{3}, L_{6}, L_{9}$ определяются точкой $p_{4}=(1: 1: 1)$, прямые $L_{2}$ и $L_{5}$ определяются точкой $p_{5} \in L_{9}$, а $L_{8}$ определяется точкой $p_{6}=$ $L_{2} \cap L_{6}$. Следовательно,

$$
\mathscr{B}_{3} \simeq\left(\mathbb{P}^{1} \backslash\{\text { три точки }\}\right) \backslash \mathscr{B}_{2}
$$

где $\mathscr{B}_{2}=\left\{\bar{L}_{4,0}\right\}$ состоит из единственной конфигурации $\bar{L}_{4,0}$, соответствующей случаю, когда $L_{3} \cap L_{5} \cap L_{8} \neq \varnothing$.

Положим $\mathscr{B}_{4}=\mathscr{B}_{4}^{\prime} \cup \mathscr{B}_{4}^{\prime \prime}$, где $\mathscr{B}_{4}^{\prime}$ (соответственно, $\mathscr{B}_{4}^{\prime \prime}$ ) состоит из конфигураций $\bar{L}_{2}^{\prime}$ (соответственно, $\left.\bar{L}_{2}^{\prime \prime}\right)$. Легко видеть, что конфигурация $\bar{L}_{2}^{\prime} \in \mathscr{B}_{4}^{\prime}$ однозначно определяется точкой $p_{5}=L_{2} \cap L_{5} \cap L_{8}$. Следовательно,

$$
\mathscr{B}_{4}^{\prime} \simeq \mathbb{P}^{2} \backslash\{\text { шесть прямых }\} .
$$

Аналогично, $\bar{L}_{2}^{\prime \prime} \in \mathscr{B}_{4}^{\prime \prime}$ однозначно определяется точкой $p_{5} \in L_{9}$ и прямой, принадлежащей пучку прямых, проходящих через точку $p_{3}$. Следовательно,

$$
\mathscr{B}_{4}^{\prime \prime} \simeq\left(\mathbb{P}^{1} \backslash\{\text { три точки }\}\right)^{2} \backslash\left(\mathscr{B}_{2} \cup \mathscr{B}_{3}\right)
$$

Как и выше, ясно, что $\bar{L}_{1} \in \mathscr{B}_{5}$ однозначно определяется прямыми $L_{2}, L_{5}$ и $L_{8}$, принадлежащими соответственно пучкам прямых, проходящих через точки $p_{1}, p_{2}$ и $p_{3}$. Следовательно,

$$
\mathscr{B}_{5} \simeq\left(\mathbb{P}^{1} \backslash\{\text { три точки }\}\right)^{3} \backslash\left(\mathscr{B}_{2} \cup \mathscr{B}_{3} \cup \mathscr{B}_{4}\right)
$$

Аналогично,

$$
\mathscr{B}_{6} \simeq\left(\mathbb{P}^{1} \backslash\{\text { три точки }\}\right)^{4} \backslash \overline{\mathscr{B}}_{5},
$$

где многообразие $\overline{\mathscr{B}}_{5}$ - это объединение вырождений конфигураций $\bar{L}_{0}, \operatorname{dim} \overline{\mathscr{B}}_{5}=3$, $\mathscr{B}_{2} \cup \mathscr{B}_{3} \cup \mathscr{B}_{4} \cup \mathscr{B}_{5} \subset \overline{\mathscr{B}}_{5}$.

УТВЕРЖДЕНИЕ 4.10. Любъе две поверхности Бурниа $X_{2}^{\prime}$ и $X_{2}^{\prime \prime}$ не изоморфньь друг другу.

ДоказАтельство. Предположим, что существует изоморфизм $h: X_{2}^{\prime} \rightarrow X_{2}^{\prime \prime}$. Тогда он индуцирует изоморфизмы $h^{*}: \operatorname{Tors}_{2}\left(X_{2}^{\prime \prime}\right) \rightarrow \operatorname{Tors}_{2}\left(X_{2}^{\prime}\right)$ и

$$
h^{*}: H^{0}\left(X_{2}^{\prime \prime}, \mathscr{O}_{X_{2}^{\prime \prime}}\left(K_{X_{2}^{\prime \prime}}+\alpha\right)\right) \rightarrow H^{0}\left(X_{2}^{\prime}, \mathscr{O}_{X_{2}^{\prime}}\left(K_{X_{2}^{\prime}}+h^{*}(\alpha)\right)\right)
$$

для каждого $\alpha \in \operatorname{Tors}_{2}\left(X_{2}^{\prime \prime}\right)$. Заметим, что из утверждения 4.8 и теоремы Римана-Роха вытекает, что $\operatorname{dim} H^{0}\left(X_{2}^{\prime \prime}, \mathscr{O}_{X_{2}^{\prime \prime}}\left(K_{X_{2}^{\prime \prime}}+\alpha\right)\right)=1$ для $\alpha \neq 0$. Следовательно, мы должны иметь

$$
K_{h^{*}(\alpha)}^{\prime}=h^{*}\left(K_{\alpha}^{\prime \prime}\right)
$$

для каждого $K_{\alpha}^{\prime \prime} \in\left|K_{X_{2}^{\prime \prime}}+\alpha\right|$. С другой стороны, среди неприводимых компонент дивизоров $K_{\alpha}^{\prime \prime}$ найдется рациональная кривая с индексом самопересечения, равным -2 (кривая $C_{9}$; см. утверждение 4.5 и предложение 4.7 ), но среди неприводимых компонент дивизоров $K_{\alpha}^{\prime} \in\left|K_{X_{2}^{\prime}}+\alpha\right|$ нет таких кривых. Противоречие. 
УТВЕРЖДЕНИЕ 4.11. Для каждого $s=0, \ldots, 4$ и для каждой конфигураиии $\bar{L}_{s, 0} \in \mathscr{B}_{6-s}$ существует лишь конечное число конфигураций $\bar{L}_{s} \in \mathscr{B}_{6-s}$, для которых соответствующие поверхности Бурниа $X_{s}=F_{s}^{-1}\left(\bar{L}_{s}\right)$ изоморфнь поверхности $X_{s, 0}=F_{s}^{-1}\left(\bar{L}_{s, 0}\right)$.

ДокАЗАТЕЛЬСТво. Положим $x=\frac{z_{1}}{z_{0}}$ и $y=\frac{z_{2}}{z_{0}}$, где $\left(z_{0}: z_{1}: z_{2}\right)$-выбранные вьше однородные координаты в $\mathbb{P}^{2}$. Тогда прямые $L_{4}, L_{3}, L_{2}, L_{7}, L_{9}, L_{8}, L_{5}$ и $L_{6}$ задаются соответственно уравнениями $x=0, x=1, x=a_{1}, y=0, y=1$, $y=b_{1}, x=c_{1} y$ и $x=c_{2} y$ при некоторых $a_{1}, b_{1}, c_{1}, c_{2} \in \mathbb{C} \backslash\{0,1\}$. Рассмотрим инъективное отображение $r_{s}: \mathscr{B}_{6-s} \rightarrow \mathbb{C}^{\operatorname{dim} \mathscr{B}_{6-s}}$, заданное следуюшим образом (если $s=2$, то рассмотрим два отображения: $r_{2}^{\prime}$ и $r_{2}^{\prime \prime}$ ).

В случае $\mathscr{B}_{3}$ имеем $c_{2}=1, c_{1}=a_{1}$ и $b_{1}=a_{1}$. Следовательно, $a_{1}$ является координатой в $\mathscr{B}_{3}$. Положим $r_{3}\left(\bar{L}_{3}\right)=a_{1} \in \mathbb{C}^{1}$. Заметим также, что конфигурация $\mathscr{B}_{2}=\left\{\bar{L}_{4,0}\right\}$ имеет координату $a_{1}=-1$.

В случае $\mathscr{B}_{4}^{\prime}$ имеем $c_{2}=1, a_{1}=b_{1} c_{1}$ и $\left(b_{1}, c_{1}\right)$ являются координатами в $\mathscr{B}_{4}^{\prime}$. Положим $r_{2}^{\prime}\left(\bar{L}_{2}^{\prime}\right)=\left(b_{1}, c_{1}\right) \in \mathbb{C}^{2}$.

Аналогично, в случае $\mathscr{B}_{4}^{\prime \prime}$ имеем $c_{2}=1, c_{1}=a_{1}$ и $\left(b_{1}, c_{1}\right)$ являются координатами в $\mathscr{B}_{4}^{\prime \prime}$. Положим $r_{2}^{\prime \prime}\left(\bar{L}_{2}^{\prime \prime}\right)=\left(b_{1}, c_{1}\right) \in \mathbb{C}^{2}$.

В случае $\mathscr{B}_{5}$ имеем $c_{2}=1$ и $\left(a_{1}, b_{1}, c_{1}\right)$ являются координатами в $\mathscr{B}_{5}$. Положим $r_{1}\left(\bar{L}_{1}\right)=\left(a_{1}, b_{1}, c_{1}\right) \in \mathbb{C}^{3}$.

В случае $\mathscr{B}_{6}$ числа $a_{1}, b_{1}, c_{1}, c_{2}$ являются координатами в $\mathscr{B}_{6}$. Положим $r_{0}\left(\bar{L}_{0}\right)=\left(a_{1}, b_{1}, c_{1}, c_{2}\right) \in \mathbb{C}^{4}$.

Очевидно, образ $r_{s}\left(\mathscr{B}_{6-s}\right)$ является открытым всюду плотным множеством B $\mathbb{C}^{\operatorname{dim} \mathscr{B}_{6-s}}$.

Пусть $\operatorname{Iso}\left(X_{s, 0}\right)$ - множество конфигураций $\bar{L}_{s}$, для которых поверхности $F_{s}^{-1}\left(\bar{L}_{s}\right)$ изоморфны поверхности $X_{s, 0}$. Заметим, что Iso $\left(X_{s, 0}\right)$ является квазипроективным подмногообразием многообразия $\mathscr{B}_{6-s}$. Действительно, каждая поверхность $X_{s}$ является поверхностью с обильным каноническим классом (возможно, по модулю $(-2)$-кривых). Вложения поверхностей $X_{s}$ в $\mathbb{P}^{10 K_{X_{s}}^{2}}$, заданные линейными системами $\left|5 K_{X_{s}}\right|$, определяет морфизм $\mu: \mathscr{B}_{6-s} \rightarrow \operatorname{Hilb}_{P_{X_{s}}}$ в схему Гильберта поверхностей в $\mathbb{P}^{10 K_{X}^{2}}$ с фиксированным многочленом Гильберта. Группа $\operatorname{PGL}\left(10 K_{X_{s}}^{2}+1, \mathbb{C}\right)$ действует на $\operatorname{Hilb}_{P_{X_{s}}}$ и

$$
\operatorname{Iso}\left(X_{s, 0}\right)=\mu^{-1}\left(\mu\left(\mathscr{B}_{6-s}\right) \cap \operatorname{PGL}\left(10 K_{X_{s}}^{2}+1, \mathbb{C}\right)\left(\mu\left(\bar{L}_{s, 0}\right)\right) .\right.
$$

Следовательно, чтобы доказать утверждение, достаточно показать, что образ $r_{s}\left(\operatorname{Iso}\left(X_{s, 0}\right)\right)$ состоит из конечного числа точек.

Для этого рассмотрим произвольные две изоморфные между собой поверхности Бурниа $X_{s, 0}, X_{s, 1}$, и пусть $h: X_{s, 0} \rightarrow X_{s, 1}$ - изоморфизм между этими поверхностями. Как и в доказательстве утверждения 4.10 , изоморфизм $h$ индуцирует изоморфизмы

$$
\begin{aligned}
h^{*}: \operatorname{Tors}_{2}\left(X_{s, 1}\right) & \rightarrow \operatorname{Tors}_{2}\left(X_{s, 0}\right) \\
h^{*}: H^{0}\left(X_{s, 1}, \mathscr{O}_{X_{s, 1}}\left(K_{X_{s, 1}}+\alpha\right)\right) & \rightarrow H^{0}\left(X_{s, 0}, \mathscr{O}_{X_{s, 0}}\left(K_{X_{s, 0}}+h^{*}(\alpha)\right)\right)
\end{aligned}
$$

для каждого $\alpha \in \operatorname{Tors}_{2}\left(X_{s, 1}\right)$. Из утверждения 4.8 и теоремы Римана-Роха вытекает, что $\operatorname{dim} H^{0}\left(X_{s, 1}, \mathscr{O}_{X_{s, 1}}\left(K_{X_{s, 1}}+\alpha\right)\right)=1$ для каждого $\alpha \neq 0$, если $s \geqslant 1$, 
и для почти всех $\alpha \neq 0$, за исключением трех частных значений $\alpha$, если $s=0$. Следовательно, мы должны иметь

$$
K_{0, h^{*}(\alpha)}=h^{*}\left(K_{1, \alpha}\right)
$$

для каждого $K_{1, \alpha} \in\left|K_{X_{s, 1}}+\alpha\right|$ (в случае $s=0$ мы рассматриваем только 60 элементов $\alpha$, для которых $\left.\operatorname{dim} H^{0}\left(X_{s, 1}, \mathscr{O}_{X_{s, 1}}\left(K_{X_{s, 1}}+\alpha\right)\right)=1\right)$. В обозначениях (4.4) каждый дивизор $K_{1, \alpha}$ является линейной комбинацией кривых $C_{j, 1}, j=1, \ldots, 9$, и $D_{i, 1}, i=1, \ldots, 3+s$. Следовательно, $h^{*}\left(R_{1}\right)=R_{0}$, где $R_{k}=\sum_{j=1}^{9} C_{j, k}+$ $\sum_{i=1}^{3+s} D_{i, k}$ для $k=0,1$ и инварианты кривых $D_{i, k}$ и $C_{j, k}$ являются инвариантами поверхностей $X_{s, k}$. В частности, множество $R^{\prime}\left(X_{s, k}\right)$, состоящее из компонент дивизора $R_{k}$, имеющих положительный род, является также таким инвариантом. Заметим, что $D_{i, k} \in R^{\prime}\left(X_{s, k}\right)$ для $i=1,2,3$ и $C_{j, k} \in R^{\prime}\left(X_{s, k}\right)$, если $t\left(L_{j, k}\right) \leqslant 2$, и, в частности, $C_{1, k}, C_{4, k}, C_{7, k} \in R^{\prime}\left(X_{s, k}\right)$.

Пусть $C$ - эллиптическая кривая. Обозначим через $B_{C}$ подмножество в $\mathbb{C} \backslash$ $\{0,1\}$, состоящее из комплексных чисел $c$ таких, что кривая $C$ может быть представлена в виде двулистного накрытия $f: C \rightarrow \mathbb{P}^{1}$, разветвленного в четырех точках $0,1, c, \infty$. Хорошо известно, что для всякой эллиптической кривой $C$ множество $B_{C}$ состоит из конечного числа точек.

Аналогично, для гиперэллиптической кривой $C, g(C)=2$, обозначим через $B_{C}$ подмножество в $(\mathbb{C} \backslash\{0,1\})^{3}$, состоящее из троек $\left(c_{1}, c_{2}, c_{3}\right)$ комплексных чисел, $c_{i} \neq c_{j}$ при $i \neq j$, таких, что кривая $C$ может быть представлена как двулистное накрытие $f: C \rightarrow \mathbb{P}^{1}$, разветвленное в шести точках $0,1, c_{1}, c_{2}, c_{3}, \infty$. Как и в случае эллиптической кривой, множество $B_{C}$ является конечным для каждой кривой $C$ рода два.

Рассмотрим ограничение накрываюшего отображения $f: X_{s} \rightarrow \widetilde{\mathbb{P}}^{2}$ на кривую $C \in R^{\prime}\left(X_{s}\right)$. Оно является двулистным накрытием прямой $\mathbb{P}^{1}$. Положим

$$
B_{X_{s}}=\bigcup_{C \in R^{\prime}\left(X_{s}\right)} B_{C} .
$$

Из приведенных выше рассуждений вытекает, что

(*) для поверхности Бурниа $X_{s}$ множсество $B_{X_{s}}$ является конечныл и является инвариантом поверхности $X_{s}$ с точностью до изоморфизма.

Теперь, чтобы завершить доказательство утверждения, достаточно заметить, что:

а) в случае $\mathscr{B}_{3}$ образ $r_{3}\left(\bar{L}_{3,0}\right)=a_{1} \in B_{X_{3,0}}$, так как $a_{1} \in B_{C_{7,0}}$;

б) в случае $\mathscr{B}_{4}^{\prime}$ (и, аналогично, в случае $\left.\mathscr{B}_{4}^{\prime \prime}\right)$ образ $r_{2}\left(\bar{L}_{2,0}\right)=\left(b_{1}, c_{1}\right)$ для некоторых $b_{1}, c_{1} \in B_{X_{2,0}}$, так как $b_{1} \in B_{C_{4,0}}$ и $c_{1} \in B_{D_{2,0}}$;

в) в случае $\mathscr{B}_{5}$ образ $r_{1}\left(\bar{L}_{1,0}\right)=\left(a_{1}, b_{1}, c_{1}\right)$ для некоторых $a_{1}, b_{1}, c_{1} \in B_{X_{1,0}}$, так как $a_{1} \in B_{C_{7,0}}, b_{1} \in B_{C_{4,0}}$ и $c_{1} \in B_{D_{2,0}}$;

г) в случае $\mathscr{B}_{6}$ образ $r_{0}\left(\bar{L}_{0,0}\right)=\left(a_{1}, b_{1}, c_{1}, c_{2}\right)$ для некоторых элементов $b_{1}$, $\left(a_{1}, c_{1}, c_{2}\right) \in B_{X_{0,0}}$, так как $b_{1} \in B_{C_{4,0}}$ и $\left(a_{1}, c_{1}, c_{2}\right) \in B_{D_{9,0}}$.

Обозначим через $\Theta_{X_{s}}$ касательный пучок и через $\Omega_{X_{s}}^{i}$ пучок дифференциальных $i$-форорм на $X_{s}$. 
ПРЕДЛОЖЕНИЕ 4.12. ДЛя $0 \leqslant s \leqslant 4$ имеем:

(i) $\operatorname{dim} H^{0}\left(X_{s}, \Theta_{X_{s}}\right)=0$;

(ii) $\operatorname{dim} H^{1}\left(X_{s}, \Theta_{X_{s}}\right)=2 s-2+3 \max (0,2-s)$;

(iii) $\operatorname{dim} H^{2}\left(X_{s}, \Theta_{X_{s}}\right)=3 \max (0,2-s)$.

ДоКАЗАТЕЛЬСтво. Известно, что $\operatorname{dim} H^{0}\left(X_{s}, \Theta_{X_{s}}\right)=0$ для поверхностей общего типа, так как группа автоморфизмов поверхностей общего типа является дискретной.

По теореме Римана-Роха эйлерова характеристика $\chi\left(\Theta_{X_{s}}\right)$ пучка $\Theta_{X_{s}}$ выражается формулой

$$
\chi\left(\Theta_{X_{s}}\right)=\sum_{i=0}^{2}(-1)^{i} \operatorname{dim} H^{i}\left(X_{s}, \Theta_{X_{s}}\right)=2 K_{X_{s}}^{2}-10=2 s-2
$$

и по двойственности Серра

$$
\operatorname{dim} H^{i}\left(X_{s}, \Theta_{X_{s}}\right)=\operatorname{dim} H^{2-i}\left(X_{s}, \Omega_{X_{s}}^{1} \otimes \Omega_{X_{s}}^{2}\right) .
$$

Следовательно, чтобы доказать предложение, достаточно доказать следующее

ПРЕДЛОЖЕНИЕ 4.13. Для поверхности Бурниа $X_{s}, s=0, \ldots, 4$, имеем

$$
\operatorname{dim} H^{0}\left(X_{s}, \Omega_{X_{s}}^{1} \otimes \Omega_{X_{s}}^{2}\right)=3 \max (0,2-s) .
$$

ДоказАтеЛЬСтво. Обозначим $X=X_{s}$. Выберем такую карту $U=\mathbb{C}^{2} \subset P^{2}$, что все особые точки конфигурации $\bar{L}$ лежат в $U$. Пусть $x, y$ - неоднородные координаты в $U, l_{i}(x, y)=0$ - уравнение прямой $L_{i} \subset \bar{L}$.

Включение полей рациональных функций $\mathbb{C}\left(\widetilde{\mathbb{P}}^{2}\right) \subset \mathbb{C}(X)$, индуцированное морфизмом $f$, является расширением Галуа с группой Галуа $G=(\mathbb{Z} / 2 \mathbb{Z})^{2}$. Пусть $\alpha_{1}=(1,0), \alpha_{2}=(0,1)$ и $\alpha_{3}=(1,1)$ - ненулевые элементы группы $G$. Отождествляя $\mathbb{C}\left(\widetilde{\mathbb{P}}^{2}\right)$ с полем $K_{0}=\mathbb{C}(x, y)$ рациональных функций от $x, y$ и поле $\mathbb{C}\left(X_{2}\right)$ с полем $K=\mathbb{C}\left(x, y, w_{1}, w_{2}, w_{3}\right)$, где $x, y, w_{1}, w_{2}, w_{3}$ удовлетворяют уравнениям (4.3), не ограничивая обшности, можем считать, что $\alpha(x)=x, \alpha(y)=y$ для всех $\alpha \in G$ и

$$
\begin{aligned}
& \alpha_{1}\left(w_{1}\right)=w_{1}, \quad \alpha_{2}\left(w_{1}\right)=\alpha_{3}\left(w_{1}\right)=-w_{1}, \\
& \alpha_{2}\left(w_{2}\right)=w_{2}, \quad \alpha_{1}\left(w_{2}\right)=\alpha_{3}\left(w_{2}\right)=-w_{2}, \\
& \alpha_{3}\left(w_{3}\right)=w_{3}, \quad \alpha_{1}\left(w_{3}\right)=\alpha_{2}\left(w_{3}\right)=-w_{3} .
\end{aligned}
$$

Положим $K_{i}=\mathbb{C}\left(x, y, w_{i}\right)$ и обозначим через $g_{i}: Z_{i} \rightarrow \mathbb{P}^{2}$ накрытие, индуцированное расширением $K_{0} \subset K_{i}$.

Рассмотрим пространства $M, M_{0}, M_{1}, M_{2}, M_{3}$ рациональных $((1,0) \otimes(2,0))$ форм на $X, \mathbb{P}^{2}, Z_{1}, Z_{2}, Z_{3}$ соответственно. Имеем $M_{0} \subset M_{i} \subset M$ для $i=1,2,3$ и

$$
\begin{aligned}
M_{0} & =\mathbb{C}(x, y) d x \otimes(d x \wedge d y) \oplus \mathbb{C}(x, y) d y \otimes(d x \wedge d y), \\
M & =K d x \otimes(d x \wedge d y) \oplus K d y \otimes(d x \wedge d y), \\
M_{i} & =K_{i} d x \otimes(d x \wedge d y) \oplus K_{i} d y \otimes(d x \wedge d y), \quad i \geqslant 1 .
\end{aligned}
$$


Более того, группа $G$ действует на $M$ и $M^{G}=M_{0}$. Кроме того, $\omega \in M$ принадлежит пространству $M_{i}, i=1,2,3$, тогда и только тогда, когда $\alpha_{i}(\omega)=\omega$ и $\alpha_{j}(\omega)=-\omega$ для $j \neq i$.

Групша Галуа $G$ действует также на пространстве $H^{0}\left(X, \Omega_{X}^{1} \otimes \Omega_{X}^{2}\right)$, и это пространство также раскладывается в прямую сумму собственных подпространств $H_{(i)}$ :

$$
H^{0}\left(X, \Omega_{X}^{1} \otimes \Omega_{X}^{2}\right)=\bigoplus_{i=0}^{3} H_{(i)}
$$

где $\omega \in H_{(i)}, i \geqslant 1$, тогда и только тогда, когда $\alpha_{i}(\omega)=\omega$ и $\alpha_{j}(\omega)=-\omega$ для $j \neq i$, a $\omega \in H_{(0)}$ тогда и только тогда, когда $\alpha_{i}(\omega)=\omega$ для всех $i$. Легко видеть, что

$$
H_{(i)}=H^{0}\left(X, \Omega_{X}^{1} \otimes \Omega_{X}^{2}\right) \cap M_{i}
$$

для $i=0,1,2,3$.

Лемма 4.14. Пусть $(V, o) \subset \mathbb{C}^{2} \times \mathbb{C}^{1}$ - росток поверхности, заданный в координатах $\left(z_{1}, z_{2}, w_{1}\right)$ уравнением $w_{1}^{2}=z_{1}$. Рассмотрим действие группь $\mathbb{Z} / 2 \mathbb{Z}$ на $H^{0}\left(V, \Omega_{V}^{1} \otimes \Omega_{V}^{2}\right)$, заданное формулами $\alpha\left(z_{1}\right)=z_{1}, \quad \alpha\left(z_{2}\right)=z_{2}$, $\alpha\left(w_{1}\right)=-w_{1}$, где $\alpha \in \mathbb{Z} / 2 \mathbb{Z}$ - ненулевой әлемент.

(i) Eсли $\omega \in H^{0}\left(V, \Omega_{V}^{1} \otimes \Omega_{V}^{2}\right)$ инвариантна при действии группы $\mathbb{Z} / 2 \mathbb{Z}$, то

$$
\omega=\left(P\left(z_{1}, z_{2}\right) \frac{d z_{1}}{z_{1}}+Q\left(z_{1}, z_{2}\right) d z_{2}\right) \otimes\left(d z_{1} \wedge d z_{2}\right)
$$

(ii) если $\omega \in H^{0}\left(V, \Omega_{V}^{1} \otimes \Omega_{V}^{2}\right)$ антиинвариантна при действии группь $\mathbb{Z} / 2 \mathbb{Z}$, mo

$$
\omega=\left(P\left(z_{1}, z_{2}\right) d z_{1}+Q\left(z_{1}, z_{2}\right) d z_{2}\right) \otimes \frac{d z_{1} \wedge d z_{2}}{w_{1}}
$$

где $P\left(z_{1}, z_{2}\right)$ и $Q\left(z_{1}, z_{2}\right)$ - некоторые аналитические функции от $z_{1}$ и $z_{2}$.

ДокАЗАТЕЛЬСТво. Заметим, что $\frac{d z_{1} \wedge d z_{2}}{w_{1}}$ является нигде не обрашаюшейся в нуль голоморфной 2-формой на $V$ и $\alpha\left(\frac{d z_{1} \wedge d z_{2}}{w_{1}}\right)=-\frac{d z_{1} \wedge d z_{2}}{w_{1}}$. Следовательно, форма $\omega \in H^{0}\left(V, \Omega_{V}^{1} \otimes \Omega_{V}^{2}\right)$ может быть записана в виде

$$
\omega=\left(h_{2} d z_{2}+h_{3} d w_{1}\right) \otimes \frac{d z_{1} \wedge d z_{2}}{w_{1}},
$$

где $h_{2}, h_{3} \in H^{0}\left(V, \mathscr{O}_{V}\right)$. Аналогично, функции $h_{i}$ могут быть записаны в виде $h_{i}=$ $H_{i}^{\prime}\left(z_{1}, z_{2}\right)+w_{1} H_{i}^{\prime \prime}\left(z_{1}, z_{2}\right)$, где $H_{i}^{\prime}\left(z_{1}, z_{2}\right)$ и $H_{i}^{\prime \prime}\left(z_{1}, z_{2}\right)$ - некоторые аналитические функции от $z_{1}$ и $z_{2}$. 
Легко видеть, что $\omega$ является инвариантной формой тогда и только тогда, когда $H_{2}^{\prime}\left(z_{1}, z_{2}\right)=H_{3}^{\prime \prime}\left(z_{1}, z_{2}\right)=0$, и $\omega$ является антиинвариантной формой тогда и только тогда, когда $H_{2}^{\prime \prime}\left(z_{1}, z_{2}\right)=H_{3}^{\prime}\left(z_{1}, z_{2}\right)=0$. Чтобы завершить доказательство леммы, заметим, что $w_{1} d w_{1}=\frac{1}{2} d z_{1}$.

УТВЕРЖДЕНИЕ 4.15. Имеем $H_{(0)}=0$.

ДокАЗАТЕЛЬСтво. Пусть $\omega \in H_{(0)}, \omega \neq 0$. Из леммы 4.14 следует, что над картой $U=\mathbb{C}^{2}$ она может быть записана в виде $\omega=\omega_{1} \otimes(d x \wedge d y)$, где $\omega_{1} \in H^{0}\left(U \backslash \operatorname{Sing} \bar{L}, \Omega_{U \backslash \operatorname{Sing}}^{1}(\log \bar{L})\right)$ является 1-формой с логарифмическими полюсами вдоль $\bar{L}$. Предположим, что она имеет полюсы вдоль прямых $L_{i_{1}}, \ldots, L_{i_{k}}$, $0 \leqslant k \leqslant 9$. Тогда $\omega$ может быть записана в виде

$$
\omega=\frac{P(x, y) d x+Q(x, y) d y}{l_{i_{1}} \ldots l_{i_{k}}} \otimes(d x \wedge d y),
$$

где $P(x, y), Q(x, y) \in \mathbb{C}[x, y]$ являются многочленами степени не больше, чем $k-4$. Действительно, $l_{i_{1}} \ldots l_{i_{k}} \omega$ является регулярной формой на $\mathbb{C}^{2} \backslash \operatorname{Sing} \bar{L}$. Следовательно, она может быть записана в виде

$$
l_{i_{1}} \ldots l_{i_{k}} \omega=(P(x, y) d x+Q(x, y) d y) \otimes(d x \wedge d y),
$$

где $P(x, y), Q(x, y) \in \mathbb{C}[x, y]$. Далее, $\omega$ является регулярной формой в обшей точке бесконечно удаленной прямой $L_{\infty}=\mathbb{P}^{1} \backslash C^{2}$. Пусть $\left(z_{0}: z_{1}: z_{2}\right)$ - такие однородные координаты на $\mathbb{P}^{2}$, что $x=\frac{z_{1}}{z_{0}}$ и $y=\frac{z_{2}}{z_{0}}$. Тогда в координатах $u=\frac{1}{x}, v=\frac{y}{x}$ форма $\omega$ имеет следуюший вид:

$\omega=\left(\left(\frac{u^{k-2} \widetilde{P}(u, v)}{u^{\operatorname{deg} P \tilde{l}_{i_{1}} \ldots \tilde{l}_{i_{k}}}}+\frac{u^{k-2} v \widetilde{Q}(u, v)}{u^{\operatorname{deg} Q \tilde{l}_{i_{1}} \ldots \tilde{l}_{i_{k}}}}\right) d u-\frac{u^{k-1} \widetilde{Q}(u, v)}{u^{\operatorname{deg} Q \tilde{l}_{i_{1}} \ldots \tilde{l}_{i_{k}}}} d v\right) \otimes\left(\frac{d u \wedge d v}{u^{3}}\right)$.

Следовательно, $\operatorname{deg} Q \leqslant k-4$, если $\omega$ является регулярной в общей точке бесконечно удаленной прямой. Точно так же мы получим, что $\operatorname{deg} P \leqslant k-4$, если рассмотрим координаты $u_{1}=\frac{1}{y}, v_{1}=\frac{x}{y}$. Следовательно, $k \geqslant 4$.

Пусть $l_{i}(x, y)=y+a_{i} x+b_{i}$. Имеем $a_{i} \neq a_{j}$, если $i \neq j$, так как все особые точки конфигурации $\bar{L}$ лежат в $U$, и

$$
d y=d l_{i}-a_{i} d x .
$$

Подставляя (4.24) в (4.23), получаем, что

$$
\omega=\frac{\left(P(x, y)-a_{i} Q(x, y)\right) d x+Q(x, y) d l_{i}}{l_{i_{1}} \ldots l_{i_{k}}} \otimes(d x \wedge d y) .
$$

Поскольку

$$
\frac{\left(P-a_{i} Q\right) d x+Q d l_{i}}{l_{i_{1}} \ldots l_{i_{k}}} \in H^{0}\left(U \backslash \operatorname{Sing} \bar{L}, \Omega_{U \backslash \operatorname{Sing} \bar{L}}^{1}\left(\log \bar{L}_{2}\right)\right),
$$

то многочлены $P(x, y)-a_{i_{j}} Q(x, y)$ должны делиться на $l_{i_{j}}(x, y)$ при $j=1, \ldots, k$. Следовательно, $k \geqslant 5$ и пучок $P(x, y)-a Q(x, y)=0$ плоских кривых степени $d=$ 
$k-4$ должен иметь $k$ различных слоев, содержащих прямые, и по предположению каждая из этих прямых не является неподвижной компонентой этого пучка.

Покажем, что это невозможно в нашем случае. Действительно, случай $d=1$ (т.е. $k=5$ ) является невозможным, так как только четыре прямые, принадлежащие конфигурации $\bar{L}$, могут лежать в одном и том же пучке. Случай $d=2$ (т.е. $k=6$ ) невозможен, так как пучок коник может иметь только три слоя, содержащих прямые.

Чтобы показать, что случай $d \geqslant 3$ (т.е. $k \geqslant 7$ ) является невозможным, заметим, что если конфигурация прямых $l_{i_{1}} \ldots l_{i_{k}}=0$ имеет четырехкратную точку $p$, то порядки нуля многочленов $P(x, y)$ и $Q(x, y)$ в этой точке должны быть не меньше двух. Следовательно, порядок нуля каждого члена пучка $P(x, y)-a Q(x, y)=0$ в точке $p$ должен быть также не меньше двух. Действительно, предположим, что конфигурация $l_{i_{1}} \ldots l_{i_{k}}=0$ имеет такую точку $p$. Не ограничивая общности, можем предполагать, что точка $p$ имеет координаты $(0,0)$. Пусть $\sigma: \widetilde{U} \rightarrow U-$ раздутие точки $p$. В одной из карт морфизм $\sigma$ задается уравнениями $x=x_{1}$ и $y=x_{1} y_{1}$. В этих новых координатах форма $\omega$ имеет следующий вид:

$$
\omega=\frac{\left(P\left(x_{1}, x_{1} y_{1}\right)+y_{1} Q\left(x_{1}, x_{1} y_{1}\right)\right) d x_{1}+x_{1} Q\left(x_{1}, x_{1} y_{1}\right) d y_{1}}{x_{1}^{4} \tilde{l}_{i_{1}} \ldots \tilde{l}_{i_{k}}} \otimes\left(x_{1} d x_{1} \wedge d y_{1}\right)
$$

и поэтому порядок нуля многочлена $Q(x, y)$ в точке $p$ должен быть не меньше двух, так как согласно лемме 4.14 форма $\omega$ является формой с логарифмическими полюсами вдоль исключительной кривой $x_{1}=0$. Аналогичные аргументы (надо рассмотреть отображение, заданное уравнениями $x=x_{2} y_{2}$ и $y=y_{2}$ ) показьвают, что порядок нуля многочлена $P(x, y)$ в точке $p$ должен быть также не меньше двух.

Покажем, что случаи $d=3,4,5$ (т.е. $k=7,8,9$ ) также невозможны, так как в каждом из этих случаев пучки кривых $P(x, y)-a Q(x, y)=0$ степени $d$ обязаны иметь неподвижную прямую, принадлежашую конфигурации прямых $l_{i_{1}} \ldots l_{i_{k}}=0$. Действительно, каждая общая точка любых двух прямых (лежаших в разных слоях пучка $P(x, y)-a Q(x, y)=0)$ из конфигурации $l_{i_{1}} \ldots l_{i_{k}}=0$ является базисной точкой пучка. Однако легко проверить, что если мы удалим любые две прямые из конфигурации $\bar{L}$ (случай $d=3$, т. е. $k=7$ ), то получим новую конфигурацию, состоящую из семи прямых таких, что найдется компонента в новой конфигурации, проходящая через четыре особые точки этой конфигурации (считаемые с кратностями). Следовательно, эта прямая должна быть неподвижной компонентой пучка кривых степени 3. Аналогично, если удалить любую прямую из конфигурации $\bar{L}$ (случай $d=4$, т. е. $k=8$ ), то получим новую конфигурацию, состоящую из восьми прямых, такую, что в новой конфигурации найдется прямая, проходящая через четырехкратную точку и через три другие особые точки новой конфигурации. В случае $d=5$ (т. е. $k=9$ ) также существует прямая (например, $L_{1}$ ), проходящая через две четырехкратные точки и две другие особые точки конфигурации $\bar{L}$.

УТВЕРЖДЕНИЕ 4.16. Пусть $X-$ поверхность Бурниа $X_{s}$. Тогда

$$
\operatorname{dim} H_{(1)}=\operatorname{dim} H_{(2)}=\operatorname{dim} H_{(3)}=\max (0,2-s) .
$$


ДокАзАтеЛЬСтво. Рассмотрим пространство $H_{(1)}$ (для $H_{(2)}$ и $H_{(3)}$ вычисление их размерностей аналогичны). Пусть $\omega \in H_{(1)}, \omega \neq 0$. Тогда, так как $\omega \in M_{1}$, имеем

$$
\omega=\left(R_{1}(x, y) d x+R_{2}(x, y) d y\right) \otimes \frac{d x \wedge d y}{w_{1}},
$$

где $R_{i}(x, y)$ являются рациональными функциями. Заметим, что форма $\frac{d x \wedge d y}{w_{1}}$ не имеет ни нулей, ни полюсов на $Y_{1} \backslash \operatorname{Sing} Y_{1}$, так как $w_{1}^{2}=l_{1} \ldots l_{6}$ и $\operatorname{deg} l_{1} \ldots l_{6}=6$ (см. $\S 3$ ). Следовательно, так как $\omega$ является регулярной формой над общей точкой прямой $L_{\infty}$, то, как и в доказательстве утверждения 4.15 , применяя лемму 4.14 , можно легко показать, что $\omega$ может быть записана в виде

$$
\omega=\frac{P(x, y) d x+Q(x, y) d y}{l_{7} l_{8} l_{9}} \otimes \frac{d x \wedge d y}{w_{1}},
$$

где $P(x, y), Q(x, y) \in \mathbb{C}[x, y]$ являются многочленами степени не больше двух и, более того, форма определяется следуюшим образом:

$$
\omega_{1}=\frac{P(x, y) d x+Q(x, y) d y}{l_{7} l_{8} l_{9}} \in H^{0}\left(U \backslash \operatorname{Sing} D_{1}, \Omega_{U \backslash \operatorname{Sing} D_{1}}^{1}\left(\log D_{1}\right)\right),
$$

где $D_{1}=L_{7}+L_{8}+L_{9}$. Не ограничивая общности, можно предполагать, что $p_{3}=(0,0), l_{1}(x, y)=x-a y, l_{7}(x, y)=y, l_{8}(x, y)=x-y$ и $l_{9}(x, y)=x$, где $a \neq 0,1$.

Чтобы разрешить особые точки поверхности $Y_{s}$, нужно раздуть четырехкратные и тройные точки конфигурации $\bar{L}_{s}$. Если форма $\omega \in H_{(1)}$, то она должна быть регулярной над раздутыми кривыми $E_{i}$.

Следующие леммы дают необходимые и достаточные условия для того, чтобы форма (4.26) была регулярной над точками кривой $E_{3}$.

ЛЕмма 4.17. Пусть $(V, o) \subset\left(\mathbb{C}^{2} \times \mathbb{C}^{2}, o\right)$ - росток нормальной поверхности, заданной в координатах $\left(z_{1}, z_{2}, w_{1}, w_{2}\right)$ уравнениями $w_{1}^{2}=x-$ ау и $w_{2}^{2}=$ $x y(x-y)$, где $a \neq 0,1$, и пусть

$$
\omega=\frac{P(x, y) d x+Q(x, y) d y}{x y(x-y)} \otimes \frac{d x \wedge d y}{w_{1}} \in H^{0}\left(\bar{V}, \Omega_{\bar{V}} \otimes \Omega_{\bar{V}}^{2}\right)
$$

где $P(x, y), Q(x, y) \in \mathbb{C}[x, y], \quad \operatorname{deg} P(x, y)=\operatorname{deg} Q(x, y)=2, u \nu: \bar{V} \rightarrow V-$ минимальное разрешение особой точки о поверхности $V$. Тогда

$$
\omega=\left(c \frac{y d x-x d y}{x y(x-y)}+\frac{P_{2}(x, y) d x+Q_{2}(x, y) d y}{x y(x-y)}\right) \otimes \frac{d x \wedge d y}{w_{1}}
$$

где с - некоторая константа и $P_{2}(x, y), Q_{2}(x, y)$ - однородные многочлены степени два. 
ДокАЗАТЕЛЬСтво. Обозначим через $Z$ образ $g(V)$ при отображении

$$
g\left(\left(x, y, w_{1}, w_{2}\right)\right)=(x, y)
$$

и пусть $\sigma: \widetilde{Z} \rightarrow Z$ - раздутие точки $g(o), E=\sigma^{-1}(g(o))$. Согласно лемме 1.4 отображение $f: \bar{V} \rightarrow \widetilde{Z}$, индуцированное отображением $g$, является аналитическим накрытием и может быть разложено в композицию $f=\bar{f}_{1} \circ h_{1}$, где $\bar{f}_{1}: \bar{V}_{1} \rightarrow \widetilde{Z}$ является $\mathbb{Z} / 2 \mathbb{Z}$-накрытием и $\bar{V}_{1}$ бимероморфно поверхности, заданной уравнением $w_{1}^{2}=x-a y$.

Морфизм $\sigma$ задается уравнениями $x=u, y=u v$ в некоторых локальных координатах на $\widetilde{Z}$. В этом случае поверхность $\bar{V}_{1}$ локально задается уравнением $w_{1}^{2}=u(1-a v)$. Следовательно, отображение $\bar{f}_{1}$ разветвлено вдоль $E$ и легко видеть, что отображение $h_{1}$ не является разветвленным в общей точке кривой $\bar{f}_{1}^{-1}(E)$. Таким образом, $h_{1}$ является локальным изоморфизмом в общей точке кривой $\bar{f}^{-1}(E)$.

Форма $\omega$ является мероморфной формой на $\bar{V}_{1}$, и по предположению ее прообраз $h^{*}(\omega)$ является голоморфной формой. Следовательно, $\omega$ является голоморфной формой в общей точке кривой $\bar{f}_{1}^{-1}(E)$. Более того, согласно лемме 4.14 она может иметь не более чем логарифмические полюсы вдоль кривых, заданных уравнениями $y=0, x-y=0$ и $x-a y=0$. Имеем

$$
\begin{aligned}
\omega & =\frac{(P(u, u v)+v Q(u, u v)) d u+u Q(u, u v) d v}{u^{3} v(1-v)} \otimes \frac{u d u \wedge d v}{w_{1}} \\
& =\left(\frac{(P(u, u v)+v Q(u, u v)) d u}{u^{2} v(1-v)}+\frac{Q(u, u v) d v}{u v(1-v)}\right) \otimes\left(d w_{1} \wedge d v\right) .
\end{aligned}
$$

Следовательно, $Q(0,0)=P(0,0)=0$ и многочлен $P(u, u v)+v Q(u, u v)$ должен делиться на $u^{2}$.

Положим $P=a_{1} x+a_{2} y+P_{2}(x, y)$ и $Q=b_{1} x+b_{2} y+Q_{2}(x, y)$, где $P_{2}$ и $Q_{2}-$ однородные многочлены степени два. Имеем

$$
P(u, u v)+v Q(u, u v)=a_{1} u+a_{2} u v+b_{1} u v+b_{2} u v^{2}+P_{2}(u, u v)+Q_{2}(u, u v)
$$

где $P_{2}(u, u v)+Q_{2}(u, u v)$ делится на $u^{2}$. Следовательно, $a_{1}=b_{2}=0$ и $a_{2}=-b_{1}$. Имеем

$$
\omega=\left(a_{2} \frac{y d x-x d y}{x y(x-y)}+\frac{P_{2}(x, y) d x+Q_{2}(x, y) d y}{x y(x-y)}\right) \otimes \frac{d x \wedge d y}{w_{1}} .
$$

Лемма 4.18. Пусть $x, y-$ координаты в $U=\mathbb{C}^{2}, \quad$ o $=(0,0), \quad D \subset U-$ дивизор, заданный уравнением $x y(x-y)=0, u$

$$
\omega_{1}=\frac{P(x, y) d x+Q(x, y) d y}{x y(x-y)} \in H^{0}\left(U \backslash\{o\}, \Omega_{U \backslash\{o\}}^{1}(\log D)\right),
$$

где $P(x, y)$ и $Q(x, y)$ - некоторые однородные многочлены степени два. Тогда $\omega_{1}$ является линейной комбиначией форм $\frac{d x}{x}, \frac{d y}{y} u \frac{d(x-y)}{x-y}$. 
ДокаЗАТЕЛЬСТво. Поскольку $\omega_{1} \in H^{0}\left(U \backslash\{(0,0)\}, \Omega_{U \backslash\{(0,0)\}}^{1}(\log D)\right)$, то $P=y P_{1}(x, y)$ и $Q=x Q_{1}(x, y)$. Пусть $P_{1}(x, y)=a_{1} x+a_{2} y$ и $Q_{1}(x, y)=b_{1} x+b_{2} y$. Положим $l=x-y$. Имеем $d l=d x-d y$. Следовательно,

$$
\begin{aligned}
\omega_{1} & =\frac{y P_{1}(x, y) d x+x Q_{1}(x, y) d y}{x y(x-y)} \\
& =\frac{\left((x-l) P_{1}(x, x-l)+x Q_{1}(x, x-l)\right) d x-x Q_{1}(x, x-l) d l}{x(x-l) l} \\
& =\frac{\left(\left(a_{1}+a_{2}+b_{1}+b_{2}\right) x^{2}+l(\ldots)\right) d x-x Q_{1}(x, y) d l}{x(x-l) l},
\end{aligned}
$$

и поэтому должно выполняться следующее тождество:

$$
a_{1}+a_{2}+b_{1}+b_{2}=0
$$

T.e.

$$
\begin{aligned}
\omega_{1} & =\frac{\left(a_{1} x y+a_{2} y^{2}\right) d x+\left(b_{1} x^{2}-\left(a_{1}+a_{2}+b_{1}\right) x y\right) d y}{x y(x-y)} \\
& =-a_{2} \frac{d x}{x}+b_{1} \frac{d y}{y}+\left(a_{1}+a_{2}\right) \frac{d(x-y)}{x-y}
\end{aligned}
$$

Из лемм $4.17,4.18$ следует, что если $\omega \in H_{(1)}$, то $\omega$ имеет следуюший вид:

$$
\omega=\left(c \frac{y d x-x d y}{y(x-y)(x-a y)}+c_{1} \frac{d x}{x}+c_{2} \frac{d y}{y}+c_{3} \frac{d(x-y)}{x-y}\right) \otimes \frac{d x \wedge d y}{w_{1}}
$$

ЛЕмма 4.19. Форма

$$
\omega_{1}=c_{1} \frac{d x}{x}+c_{2} \frac{d y}{y}+c_{3} \frac{d(x-y)}{x-y}
$$

является регулярной в общей точке бесконечно удаленной прямой $L_{\infty}=$ $\mathbb{P}^{2} \backslash \mathbb{C}^{2}$ тогда и только тогда, когда $c_{1}+c_{2}+c_{3}=0$.

ДокАЗАТЕЛЬСТво. Пусть $x=\frac{1}{u}$ и $y=\frac{v}{u}$. Имеем

$$
\begin{aligned}
\omega_{1} & =c_{1} \frac{d x}{x}+c_{2} \frac{d y}{y}+c_{3} \frac{d(x-y)}{x-y} \\
& =\left(c_{1}+c_{2}+c_{3}\right) \frac{d u}{u}+\frac{c_{2}(1-v)-c_{3} v}{v(1-v)} d v .
\end{aligned}
$$

Лемма 4.20. Пусть $D \subset \mathbb{P}^{2}$ - проективное замыкание кривой $D_{0} \subset \mathbb{C}^{2}$, заданной уравнением $x y(x-y)=0$, и пусть о $=(0,0) \in \mathbb{C}^{2}-$ начало координат. Тогда

$$
\frac{y d x-x d y}{x y(x-y)} \in H^{0}\left(\mathbb{P}^{2} \backslash\{o\}, \Omega_{\mathbb{P}^{2} \backslash\{o\}}^{1}(\log D)\right) .
$$


ДОКАЗАТЕЛЬСТВО проводится прямой проверкой.

Из лемм 4.19 и 4.20 следует, что если форма $\omega$, записанная в виде (4.27), принадлежит пространству $H_{(1)}$, то

$$
c_{1}+c_{2}+c_{3}=0
$$

Пусть точка $p_{2}$ имеет координаты $(b, 0)$. Следуюшая лемма дает для формы (4.27) необходимое и достаточное условие ее регулярности над точками кривой $E_{2}$.

ЛЕмма 4.21. Пусть $\bar{V}$ - десингуляризация ростка поверхности $(V, o) \subset$ $\left(\mathbb{C}^{2} \times \mathbb{C}^{2}, 0\right)$, заданной в координатах $\left(x, y, w_{1}, w_{3}\right)$ уравнениями $w_{1}^{2}=\left(x-a_{1} y\right) \times$ $\left(x-a_{2} y\right)\left(x-a_{3} y\right) u w_{2}^{3}=y, u$ nycms

$$
\omega=\left(c \frac{y d x-(x+b) d y}{(x+b) y(x-y+b)}+c_{2} \frac{d y}{y}\right) \otimes \frac{d x \wedge d y}{w_{1}} \in H^{0}\left(\bar{V}, \Omega_{\bar{V}}^{1} \otimes \Omega_{\bar{V}}^{2}\right),
$$

где $a_{1}, a_{2}, a_{3}$ и $b$-некоторье константьи, $a_{i} \neq a_{j}$ при $i \neq j, a_{i} \neq 0$ для $i=1,2,3 u b \neq 0$. Тогдa $c-b c_{1}=0$.

ДоказАтеЛЬСтво. Рассмотрим накрытие $g: V \rightarrow g(V)=Z \subset \mathbb{C}^{2}$, заданное формулой $g\left(\left(x, y, w_{1}, w_{3}\right)\right)=(x, y)$, и пусть $\sigma: \widetilde{Z} \rightarrow Z$ - раздутие точки $g(o)=(0,0), E=\sigma^{-1}(g(o))$. Согласно лемме 1.4 отображение $f: \bar{V} \rightarrow \widetilde{Z}$, индуцированное отображением $g$, является регулярным накрытием, и оно может быть разложено в композицию $f=\bar{f}_{1} \circ h_{1}$, где $\bar{f}_{1}: \bar{V}_{1} \rightarrow \widetilde{Z}$ является $\mathbb{Z} / 2 \mathbb{Z}$-накрытием и поверхность $\bar{V}_{1}$ бимероморфна поверхности, заданной уравнением $w_{1}^{2}=\left(x-a_{1} y\right) \times$ $\left(x-a_{2} y\right)\left(x-a_{3} y\right)$.

Пусть морфизм $\sigma$ задается уравнениями $x=u v, y=v$ в некоторых локальных координатах на $\widetilde{Z}$. Тогда поверхность $\bar{V}_{1}$ локально задается уравнением $\widetilde{w}_{1}^{2}=$ $v\left(u-a_{1}\right)\left(u-a_{2}\right)\left(u-a_{3}\right)$, где $\widetilde{w}_{1}=\frac{w_{1}}{v}$, и поверхность $\bar{V}-$ это нормализация поверхности, заданной локально уравнениями $\widetilde{w}_{1}^{2}=v\left(u-a_{1}\right)\left(u-a_{2}\right)\left(u-a_{3}\right)$ и $\widetilde{w}_{3}^{2}=v$. Следовательно, отображение $\bar{f}_{1}$ разветвлено вдоль исключительной кривой $E$, заданной уравнением $v=0$, а $h_{1}$ не разветвлено в общей точке кривой $\bar{f}_{1}^{-1}(E)$. Таким образом, $h_{1}$ является локальным изоморфизмом в общей точке кривой $f^{-1}(E)$.

Имеем

$$
\begin{aligned}
\omega & =\left(c \frac{y d x-(x+b) d y}{(x+b) y(x-y+b)}+c_{2} \frac{d y}{y}\right) \otimes \frac{d x \wedge d y}{w_{1}} \\
& =\frac{c v^{2} d u+\left(-c b+c_{2} b^{2}+c_{2} v(\ldots)\right) d v}{v(u v+b)(u v-v+b)} \otimes \frac{d u \wedge d v}{\widetilde{w}_{1}}
\end{aligned}
$$

Поскольку $\omega \in H^{0}\left(\bar{V}, \Omega \frac{1}{V} \otimes \Omega_{\bar{V}}^{2}\right)$ и $b \neq 0$, то из леммы 4.14 следует, что разность $c-c_{2} b$ должна быть равна нулю.

Из леммы 4.21 следует, что если форма $\omega$, имеющая вид (4.27), принадлежит пространству $H_{(1)}$, то

$$
c-b c_{2}=0,
$$

где $p_{2}=(0, b)$.

Можно проверить, что точка $p_{1}$ не дает ограничений на форму (4.27). 
Посмотрим, какие ограничения на форму (4.27) дают тройные точки. Пусть одна из прямых $L_{8}$ или $L_{9}$ (скажем, $L_{9}$ ) проходит через тройную точку $p_{3+i}$ конфигурации $\bar{L}_{s}$. Так как точка $p_{3+i} \in L_{9}$, то она имеет координаты $\left(0, b_{1}\right), b_{1} \neq 0$.

ЛЕмма 4.22. Пусть $\bar{V}$ - десингуляризация ростка поверхности $(V, o) \subset$ $\left(\mathbb{C}^{2} \times \mathbb{C}^{2}, o\right)$, заданной в координатах $\left(x, y, w_{1}, w_{2}\right)$ уравнениями $w_{1}^{2}=\left(x-a_{1} y\right) \times$ $\left(x-a_{2} y\right)$ и $w_{2}^{2}=x\left(x-a_{1} y\right)$, и пусть форма

$$
\omega=\left(c \frac{\left(y+b_{1}\right) d x-x d y}{x\left(y+b_{1}\right)\left(x-y-b_{1}\right)}+c_{1} \frac{d x}{x}\right) \otimes \frac{d x \wedge d y}{w_{1}}
$$

принадлежит пространству $H^{0}\left(\bar{V}, \Omega \frac{1}{\bar{V}} \otimes \Omega_{\bar{V}}^{2}\right)$, где $a_{1}, a_{2}$ и $b_{1}$ - некоторье константы $, a_{1} \neq a_{2}, a_{i} \neq 0, b_{1} \neq 0$. Тогда $c-b_{1} c_{1}=0$.

ДокАЗАТЕЛЬСТво. Как и в доказательстве леммы 4.17, рассмотрим отображение $g: V \rightarrow g(V)=Z \subset \mathbb{C}^{2}$, заданное формулой

$$
g\left(\left(x, y, w_{1}, w_{2}\right)\right)=(x, y)
$$

и пусть $\sigma: \widetilde{Z}^{2} \rightarrow Z$ - раздутие точки $g(o)=(0,0), E=\sigma^{-1}(g(o))$. Согласно лемме 1.4 отображение $f: \bar{V} \rightarrow \widetilde{Z}$, индуцированное отображением $g$, является регулярньм накрытием, и оно может быть разложено в композицию $f=\bar{f}_{1} \circ h_{1}$, где $\bar{f}_{1}: \bar{V}_{1} \rightarrow \widetilde{Z}$ является $\mathbb{Z} / 2 \mathbb{Z}$-накрытием и поверхность $\bar{V}_{1}$ бимероморфна поверхности, заданной уравнением $w_{1}^{2}=\left(x-a_{1} y\right)\left(x-a_{2} y\right)$.

Пусть $\sigma$ задано уравнениями $x=u, y=u v$ в некоторых локальных координатах на $\widetilde{Z}$. Тогда поверхность $\bar{V}_{1}$ локально задается уравнением $\widetilde{w}_{1}^{2}=\left(1-a_{1} v\right)\left(1-a_{2} v\right)$, где $\widetilde{w}_{1}=\frac{w_{1}}{u}$, и поверхность $\bar{V}$ является нормализацией поверхности, заданной локально уравнениями $\widetilde{w}_{1}^{2}=\left(1-a_{1} v\right)\left(1-a_{2} v\right)$ и $\widetilde{w}_{2}^{2}=\left(1-a_{1} v\right)$, где $\widetilde{w}_{2}=\frac{w_{2}}{u}$. Следовательно, $\bar{f}_{1}$ не разветвлено вдоль исключительной кривой $E$, заданной уравнением $u=0$, и $h_{1}$ не разветвлено в общей точке кривой $\bar{f}_{1}^{-1}(E)$. Таким образом, $h_{1}$ является локальным изоморфизмом в общей точке кривой $f^{-1}(E)$.

Имеем

$$
\begin{aligned}
\omega & =\left(c \frac{\left(y+b_{1}\right) d x-x d y}{x\left(y+b_{1}\right)\left(x-y-b_{1}\right)}+c_{1} \frac{d x}{x}\right) \otimes \frac{d x \wedge d y}{w_{1}} \\
& =\frac{c b_{1}-c_{1} b_{1}^{2}+u(\ldots) d u+u(\ldots) d v}{u\left(u v+b_{1}\right)\left(u-u v-b_{1}\right)} \otimes \frac{d u \wedge d v}{\widetilde{w}_{1}} .
\end{aligned}
$$

Поскольку $\omega \in H^{0}\left(\bar{V}, \Omega_{\bar{V}}^{\frac{1}{V}} \otimes \Omega_{\bar{V}}^{2}\right)$ и $b_{1} \neq 0$, то число $c-c_{1} b_{1}$ должно быть равно нулю.

Из леммы 4.22 следует, что если форма $\omega$, записанная в виде (4.27), принадлежит пространству $H_{(1)}$, то

$$
c-c_{1} b_{1}=0
$$

где $p_{3+i}=\left(0, b_{1}\right) \in L_{9}$.

Если конфигурация $\bar{L}$ имеет две тройные точки с координатами $\left(0, b_{1}\right)$ и $\left(0, b_{2}\right)$, лежащие на прямой $L_{9}$, то уравнения $c-c_{1} b_{1}=0$ и $c-c_{1} b_{2}=0$ являются линейно 
независимыми. Аналогично, легко проверить, что если тройная точка $p_{s+3} \in L_{8}$, то она также дает некоторое линейное уравнение вида

$$
f\left(c, c_{3}\right)=0
$$

В качестве следствия получаем, что пространство $H_{(1)}$ состоит из форм

$$
\omega=\left(c \frac{y d x-x d y}{x y(x-y)}+c_{1} \frac{d x}{x}+c_{2} \frac{d y}{y}+c_{3} \frac{d(x-y)}{x-y}\right) \otimes \frac{d x \wedge d y}{w_{1}}
$$

удовлетворяющих $2+s$ линейным уравнениям (4.28)-(4.31). Отметим, что эти уравнения являются линейно независимыми. Следовательно, $\operatorname{dim} H_{(1)}=$ $\max (0,2-s)$.

Обозначим через $\mathscr{M}_{6-s}, 0 \leqslant s \leqslant 4$, объединение неприводимых компонент схемы модулей поверхностей Бурниа типа $s$ и через $\widetilde{\mathscr{B}}_{6-s}$ образ многообразия $\mathscr{B}_{6-s}$ в $\mathscr{M}_{6-s}$.

СлЕДСТВИЕ 4.23. Многообразие $\widetilde{\mathscr{B}}_{6-s}$ является всюду плотным в $\mathscr{M}_{6-s}$, если $s \leqslant 2$, и, кроме того, имеет место:

(i) пространство $\mathscr{M}_{2}$ является неособим в точке $X_{4,0}=\widetilde{\mathscr{B}}_{2}, \operatorname{dim} \mathscr{M}_{2}=6$;

(ii) пространство $\mathscr{M}_{3}$ является неособим во всех точках $X_{3} \in \widetilde{\mathscr{B}}_{3}$, $\operatorname{dim} \mathscr{M}_{3}=4$, и $\widetilde{\mathscr{B}}_{3}$ является рациональной кривой;

(iii) пространство $\mathscr{M}_{4}=M_{4}^{\prime} \cup M_{4}^{\prime \prime}$ coстоит из двух неприводимых рациональных поверхностей $\mathscr{M}_{4}^{\prime}$ и $\mathscr{M}_{4}^{\prime \prime}, \mathscr{M}_{4}$ не особо в каждой точке $X_{2} \in \widetilde{\mathscr{B}}_{4}$;

(iv) пространство $\mathscr{M}_{5}$ является унирациональным трехмерным многообразием, неособым в каждой точке $X_{1} \in \widetilde{\mathscr{B}}_{5}$;

(v) пространство $\mathscr{M}_{6}$ является унирациональным четырехмерным многообразием, неособим в каждой точке $X_{0} \in \widetilde{\mathscr{B}}_{6}$.

ДокАЗАТЕЛЬСТво следует из (4.17)-(4.21), утверждений 4.10, 4.11 и предложения 4.12.

ПРЕДЛОЖЕНИЕ 4.24. В обозначениях, сделанных в $n .4 .1$, пусть накрытие Кампеделли $f_{C}: X_{C} \rightarrow \widetilde{\mathbb{P}}^{2}$ разветвлено вдоль конфигурации Кампеделли, изображсенной на рис. 6. Тогда поверхность Бурниа $X_{4}$ изоморфна поверхности Кампеделли $X_{C}$.

ДокАЗАТЕЛьСтво. Прежде всего заметим, что накрытие $f_{C}$ может быть разложено в композицию $f_{C}=f_{C, 1} \circ h_{C, 1}$, где $\bar{f}_{C, 1}: X_{C, 1} \rightarrow \widetilde{\mathbb{P}}^{2}$ является десингуляризацией двойного накрытия $g_{C, 1}: Y_{C, 1} \rightarrow \mathbb{P}^{2}$, заданного в неоднородных координатах уравнением

$$
w_{1}^{2}=l_{(1,0,0)} l_{(1,1,0)} l_{(1,0,1)} l_{(1,1,1)}
$$

(здесь $l_{\alpha}(x, y)=0$ - уравнение прямой $\left.L_{\alpha}\right)$. Чтобы разрешить особые точки поверхности $Y_{C, 1}$, надо раздуть точки $p_{1}, \ldots, p_{6}$. Пусть $\sigma: \widetilde{\mathbb{P}}^{2} \rightarrow \mathbb{P}^{2}-$ композиция этих раздутий, $E_{i}=\sigma^{-1}\left(p_{i}\right)$ и $\widetilde{L}_{\alpha}=\sigma^{-1}\left(L_{\alpha}\right)$.

Легко видеть, что кривые $E_{i}$ не принадлежат дивизору ветвления накрытия $f_{C, 1}$ и для каждого элемента $\left(0, a_{2}, a_{3}\right)$ собственный прообраз $f_{C, 1}^{-1}\left(\widetilde{L}_{\left(0, a_{2}, a_{3}\right)}\right)$ кривой $\widetilde{L}_{\left(0, a_{2}, a_{3}\right)}$ является несвязным объединением двух рациональных кривых 


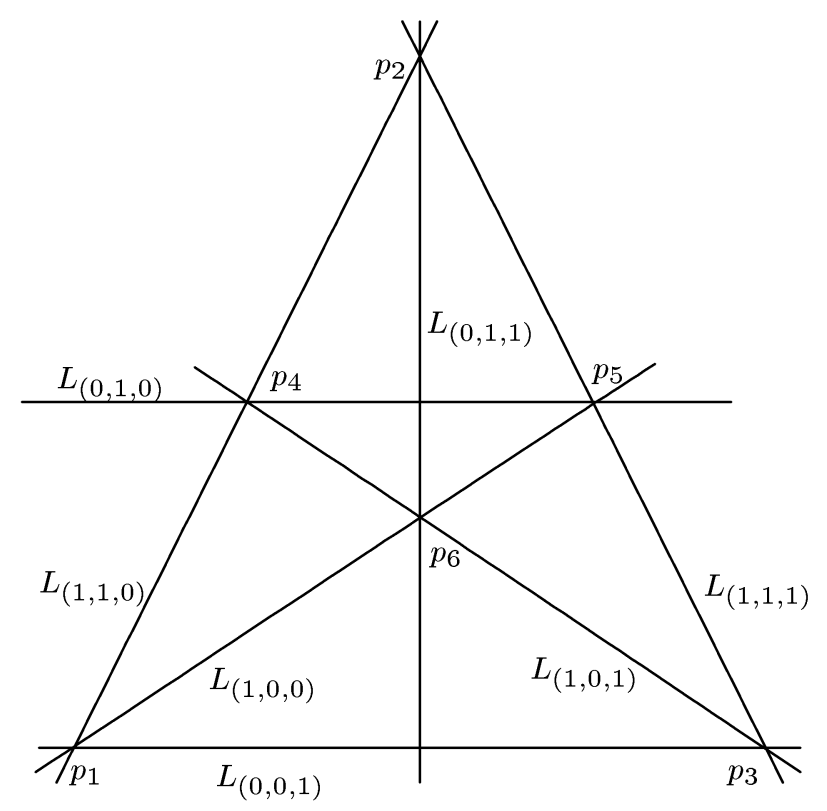

Рис.6

$L_{\left(0, a_{2}, a_{3}\right)}^{\prime}$ и $L_{\left(0, a_{2}, a_{3}\right)}^{\prime \prime}$, так как рациональная кривая $\widetilde{L}_{\left(0, a_{2}, a_{3}\right)}$ не пересекается с дивизором ветвления накрытия $f_{C, 1}$. Следовательно, $(\mathbb{Z} / 2 \mathbb{Z})^{2}$-накрытие $h_{C, 1}$ : $X_{C} \rightarrow X_{C, 1}$ разветвлено над объединением кривых $E_{i}, i=1, \ldots, 6$, и кривых $f_{C, 1}^{-1}\left(\widetilde{L}_{\left(0, a_{2}, a_{3}\right)}\right),\left(a_{2}, a_{3}\right) \in(\mathbb{Z} / 2 \mathbb{Z})^{2}$. Имеем

$$
\left(L_{\left(0, a_{2}, a_{3}\right)}^{\prime}, L_{\left(0, a_{2}, a_{3}\right)}^{\prime}\right)_{X_{C, 1}}=\left(L_{\left(0, a_{2}, a_{3}\right)}^{\prime \prime}, L_{\left(0, a_{2}, a_{3}\right)}^{\prime \prime}\right)_{X_{C, 1}}=-1
$$

так как индекс самопересечения $\left(\widetilde{L}_{\left(0, a_{2}, a_{3}\right)}, \widetilde{L}_{\left(0, a_{2}, a_{3}\right)}\right)_{\widetilde{\mathbb{P}}^{2}}=-1$ и $\operatorname{deg} f_{C, 1}=2$. Совместная конфигурация кривых $L_{\left(0, a_{2}, a_{3}\right)}^{\prime}, L_{\left(0, a_{2}, a_{3}\right)}^{\prime \prime},\left(a_{2}, a_{3}\right) \in(\mathbb{Z} / 2 \mathbb{Z})^{2}$, изображена на рис. 7.

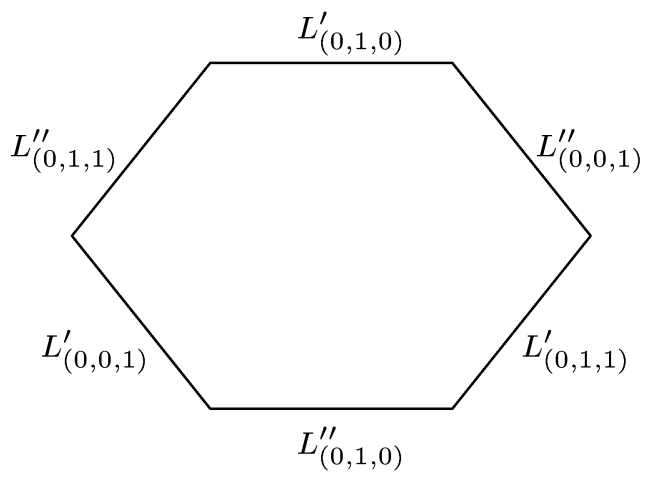

Рис. 7 
Аналогично, для каждого $\left(1, a_{2}, a_{3}\right)$ индекс самопересечения

$$
\left(\widetilde{L}_{\left(1, a_{2}, a_{3}\right)}, \widetilde{L}_{\left(1, a_{2}, a_{3}\right)}\right)_{\widetilde{\mathbb{P}}^{2}}=-2,
$$

и, следовательно, собственный прообраз $D_{\left(1, a_{2}, a_{3}\right)}=f_{C, 1}^{-1}\left(\widetilde{L}_{\left(1, a_{2}, a_{3}\right)}\right)$ имеет индекс самопересечения

$$
\left(D_{\left(1, a_{2}, a_{3}\right)}, D_{\left(1, a_{2}, a_{3}\right)}\right)_{X_{C, 1}}=\left(\frac{1}{2} f_{C, 1}^{*}\left(\widetilde{L}_{\left(1, a_{2}, a_{3}\right)}\right), \frac{1}{2} f_{C, 1}^{*}\left(\widetilde{L}_{\left(1, a_{2}, a_{3}\right)}\right)\right)_{X_{C, 1}}=-1
$$

а также

$$
\left(D_{\left(1, a_{2}, a_{3}\right)}, L_{\left(0, b_{2}, b_{3}\right)}^{\prime}\right)_{X_{C, 1}}=\left(D_{\left(1, a_{2}, a_{3}\right)}, L_{\left(0, b_{2}, b_{3}\right)}^{\prime \prime}\right)_{X_{C, 1}}=0
$$

для всех $\left(a_{2}, a_{3}\right)$ и $\left(b_{2}, b_{3}\right)$. Заметим, что каждая $D_{\left(1, a_{2}, a_{3}\right)}$ также является рациональной кривой.

Нетрудно видеть, что $X_{C, 1}$ является рациональной поверхностью, и если $\tau$ : $X_{C, 1} \rightarrow \widetilde{X}_{C, 1}-$ стягивание кривых $L_{(0,1,0)}^{\prime}, L_{(0,0,1)}^{\prime}, L_{(0,1,1)}^{\prime}$ и четырех кривых $D_{\left(1, a_{2}, a_{3}\right)}$ в точки, то $\widetilde{X}_{C, 1}$ изоморфна проективной плоскости $\mathbb{P}^{2}$, а образ

$$
\tau\left(\sum_{i=1}^{6} E_{i}+L_{(0,1,0)}^{\prime \prime}+L_{(0,0,1)}^{\prime \prime}+L_{(0,1,1)}^{\prime \prime}\right)
$$

является конфигурацией Бурниа $\bar{L}_{4}$ и накрытие $h_{C, 1}$ совпадает с накрытием Бурниа $f: X_{4} \rightarrow \widetilde{\mathbb{P}}^{2}$.

СЛЕДСТВИЕ 4.25. Пространство модулей $\mathscr{M}_{2}$ совпадает с пространством модулей $\mathscr{C}$ поверхностей Кампеделли.

4.3. Поверхности $X$ общего типа с $p_{g}=0, K_{X}^{2}=6$ и $(\mathbb{Z} / 3 \mathbb{Z})^{3} \subset$ $\operatorname{Tors}(X)$. Пусть $\bar{L}=L_{1}+\cdots+L_{6}-$ конфигурация шести прямых в $\mathbb{P}^{2}$, имеющая три тройные точки $p_{1}, p_{2}, p_{3}$, не лежашие на одной прямой. Такая конфигурация $\bar{L}$ изображена на рис. 8.

Рассмотрим накрытие $g: Y \rightarrow \mathbb{P}^{2}$, ассоциированное с эпиморфизмом $\varphi$ : $H_{1}\left(\mathbb{P}^{2} \backslash \bar{L}, \mathbb{Z}\right) \rightarrow G=(\mathbb{Z} / 3 \mathbb{Z})^{2}$, заданным формулами

$$
\begin{aligned}
& \varphi\left(\lambda_{1}\right)=\varphi\left(\lambda_{2}\right)=\varphi\left(\lambda_{3}\right)=(1,0), \\
& \varphi\left(\lambda_{4}\right)=(2,1), \quad \varphi\left(\lambda_{5}\right)=(1,1), \quad \varphi\left(\lambda_{6}\right)=(0,1) .
\end{aligned}
$$

Поверхность $Y$ имеет три особые точки, лежашие над тройными точками $p_{i}$. Согласно лемме 1.4 , чтобы разрешить эти особые точки, достаточно раздуть точки $p_{i}$ и рассмотреть индуцированное накрытие Галуа $f: X \rightarrow \widetilde{\mathbb{P}}^{2}$. Пусть $\sigma: \widetilde{\mathbb{P}}^{2} \rightarrow \mathbb{P}^{2}-$ композиция раздутий точек $p_{i}$. Обозначим через $E_{i}=\sigma^{-1}\left(p_{i}\right)$ исключительную кривую, лежащую над точкой $p_{i}$. 


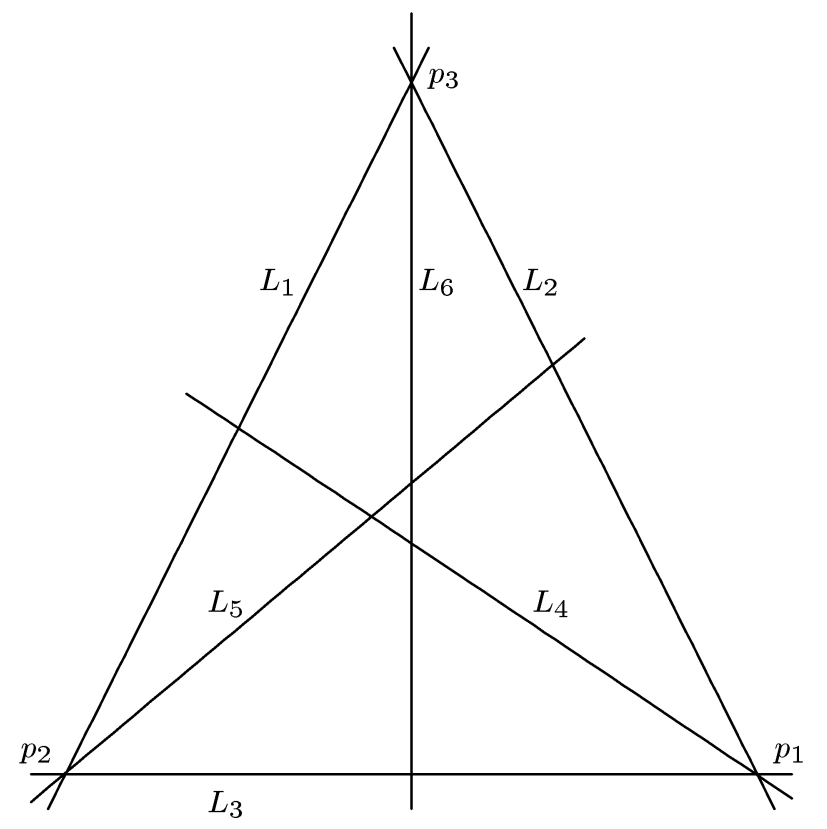

Рис. 8

ПРЕДЛОЖЕНИЕ 4.26. Построенная выше поверхность $X$ является поверхностью общего типа с $K_{X}^{2}=6, p_{g}=0 u(\mathbb{Z} / 3 \mathbb{Z})^{3} \subset \operatorname{Tors}(X)$.

ДокАЗАТЕЛЬСТво. Согласно утверждению 2.2 имеем $3 K_{X}=\left|f^{*}\left(3 L-\sum E_{i}\right)\right|$, где $L=\sigma^{*}\left(\mathbb{P}^{1}\right)$ - полньй прообраз прямой $\mathbb{P}^{1} \subset \mathbb{P}^{2}$. Следовательно, $X$ является поверхностью общего типа с обильным каноническим классом. Из (2.2) и (2.8) легко видеть, что $K_{X}^{2}=6$ и $e(X)=6$. Следовательно, согласно формуле Нётера имеем $p_{a}=1-q+p_{g}=1$. Как и выше, чтобы вычислить $p_{g}$, достаточно вычислить геометрические роды четырех циклических накрытий, ассоциированных с четырьмя эпиморфизмами из группы $G=(\mathbb{Z} / 3 \mathbb{Z})^{2}$ в циклическую группу $\mathbb{Z} / 3 \mathbb{Z}$. Эти накрытия задаются в неоднородных координатах соответственно следующими уравнениями:

$$
\begin{aligned}
& w_{1}^{3}=l_{1} l_{2} l_{3} l_{4}^{2} l_{5}, w_{2}^{3}=l_{1} l_{2} l_{3} l_{5}^{2} l_{6}, \\
& w_{3}^{3}=l_{1} l_{2} l_{3} l_{4} l_{6}^{2}, \quad w_{4}^{3}=l_{4} l_{5} l_{6} .
\end{aligned}
$$

Применяя утверждение 3.3 , легко проверить, что геометрический род каждого из этих накрытий равен нулю. Следовательно, поверхность $X$ имеет геометрический род $p_{g}=0$.

Чтобы увидеть, что $(\mathbb{Z} / 3 \mathbb{Z})^{3} \subset \operatorname{Tors}(X)$, рассмотрим универсальное накрытие $g_{u(3)}: \widetilde{Y}_{u(3)} \rightarrow \widetilde{\mathbb{P}}^{2}$, соответствуюшее эпиморфизму

$$
\bar{\varphi}: H_{1}\left(\mathbb{P}^{2} \backslash \bar{L}, \mathbb{Z}\right) \rightarrow H_{1}\left(\mathbb{P}^{2} \backslash \bar{L}, \mathbb{Z} / 3 \mathbb{Z}\right) \simeq(\mathbb{Z} / 3 \mathbb{Z})^{5}
$$


заданному формулами

$$
\begin{array}{ll}
\varphi\left(\lambda_{1}\right)=(1,0,0,0,0), \quad \varphi\left(\lambda_{2}\right)=(1,0,1,0,0), \\
\varphi\left(\lambda_{3}\right)=(1,0,0,1,0), \quad \varphi\left(\lambda_{4}\right)=(2,1,0,0,1), \\
\varphi\left(\lambda_{5}\right)=(1,1,0,0,0), \quad \varphi\left(\lambda_{6}\right)=(0,1,2,2,2) .
\end{array}
$$

Легко видеть, что накрытие Галуа $h_{u, \varphi}: X_{u} \rightarrow X$, индуцированное проекцией $\psi:(\mathbb{Z} / 3 \mathbb{Z})^{5} \rightarrow G=(\mathbb{Z} / 3 \mathbb{Z})^{2}$ на первые две координаты, является неразветвленным. Следовательно, согласно следствию 1.6 имеем включение $(\mathbb{Z} / 3 \mathbb{Z})^{3} \subset$ Tors $X$.

УТВЕРЖДЕНИЕ 4.27. Иррегулярность поверхности $X_{u}$ равна $q\left(X_{u}\right)=3$.

ДокАЗАТЕЛьСтво. Как и в доказательстве утверждения 4.8, чтобы вычислить $q$, достаточно вычислить $p_{a}$ и $p_{g}$.

Имеем $p_{a}(X)=1$. Следовательно, арифметический род $p_{a}\left(X_{u}\right)=3^{3}$, так как $h_{u, \varphi}$ является неразветвленным накрытием и $\operatorname{deg} h_{u, \varphi}=3^{3}$.

Согласно утверждению 3.3 , чтобы вычислить $p_{g}$, достаточно вычислить геометрические роды $\frac{3^{5}-1}{2}=121$ циклических накрытий, соответствующих $\frac{3^{5}-1}{2}$ эпиморфизмам $\psi_{m}, m=1, \ldots, 121$, группы $G_{u, \varphi}=(\mathbb{Z} / 3 \mathbb{Z})^{5}$ в циклическую группу $\mathbb{Z} / 3 \mathbb{Z}$. Эти вычисления оставлены читателю, заметим лишш, что вклад в иррегулярность поверхности $X_{u}$ дают только следуюшие циклические накрытия:

$$
z_{1}^{3}=l_{1} l_{2} l_{6}, \quad z_{2}^{3}=l_{1} l_{3} l_{5}, \quad z_{3}^{3}=l_{2} l_{3} l_{4} .
$$

СлЕДСТВИЕ 4.28. Фундаментальная группа построенной выше поверхности $X$ является бесконечной неабелевой группой.

ДоКАЗАТЕЛЬСТво следует из утверждения 4.27.

4.4. Поверхности Годо. Пусть $\bar{L}=L_{1}+L_{2}+L_{3}+L_{4}$-конфигурация четырех прямых в $\mathbb{P}^{2}$, находящихся в общем положении. Рассмотрим следующие накрытия: универсальное накрытие $g_{u(5)}: Y_{u(5)} \rightarrow \mathbb{P}^{2}$, соответствующее эпиморфизму

$$
\bar{\varphi}: H_{1}\left(\mathbb{P}^{2} \backslash \bar{L}, \mathbb{Z}\right) \rightarrow H_{1}\left(\mathbb{P}^{2} \backslash \bar{L}, \mathbb{Z} / 5 \mathbb{Z}\right) \simeq(\mathbb{Z} / 5 \mathbb{Z})^{3}
$$

накрытие $g: Y \rightarrow \mathbb{P}^{2}$, ассоциированное с эпиморфизмом $\varphi: H_{1}\left(\mathbb{P}^{2} \backslash L, \mathbb{Z}\right) \rightarrow(\mathbb{Z} / 5 \mathbb{Z})^{2}$, заданным (в некоторых координатах в групе $G=(\mathbb{Z} / 5 \mathbb{Z})^{2}$ ) формулами

$$
\varphi\left(\lambda_{1}\right)=(1,0), \quad \varphi\left(\lambda_{2}\right)=(0,1), \quad \varphi\left(\lambda_{3}\right)=(1,2), \quad \varphi\left(\lambda_{4}\right)=(3,2),
$$

и накрытие $h: Y_{u(5)} \rightarrow Y$, соответствующее эпиморфизму $\psi:(\mathbb{Z} / 5 \mathbb{Z})^{3} \rightarrow G=$ $(\mathbb{Z} / 5 \mathbb{Z})^{2}$ такому, что $\varphi=\psi \circ \bar{\varphi}$. Из леммы 1.4 следует, что поверхность $Y$ является неособой, и согласно предложению 1.5 накрытие $h$ является неразветвленным.

ПРЕДЛОЖЕНИЕ 4.29. Построенная выше поверхность $Y$ является поверхностью общего типа с $K_{Y}^{2}=1, p_{g}=0 u \operatorname{Tors}(Y)=\mathbb{Z} / 5 \mathbb{Z}$. 
ДоказАТЕЛЬСтво. Согласно утверждению 2.2 имеем $5 K_{Y}=\left|f^{*}(L)\right|$, где $L-$ прямая в $\mathbb{P}^{2}$. Следовательно, $Y$ является поверхностью общего типа с обильным каноническим классом. Применив $(2.2)$ и $(2.3)$, легко видеть, что $K_{Y}^{2}=1$ и $e(Y)=11$. Следовательно, согласно формуле Нётера, $p_{a}=1-q+p_{g}=1$. Чтобы вычислить $p_{g}$, достаточно вычислить геометрические роды шести циклических накрытий, ассоциированных с шестью циклическими подгруппами в группе $G$ и заданных соответственно следуюшими уравнениями:

$$
\begin{aligned}
& w_{1}^{5}=l_{1} l_{3} l_{4}^{3}, \quad w_{2}^{5}=l_{2} l_{3}^{2} l_{4}^{2}, \quad w_{3}^{5}=l_{1} l_{2} l_{3}^{3}, \\
& w_{4}^{5}=l_{1}^{2} l_{2} l_{3}^{4} l_{4}^{3}, \quad w_{5}^{5}=l_{1} l_{2}^{2} l_{4}^{2}, \quad w_{6}^{5}=l_{1} l_{2}^{3} l_{3}^{2} l_{4}^{4} .
\end{aligned}
$$

Применяя вычисления, проведенные в $\S 3$, можно легко проверить, что геометрический род каждого из этих накрытий равен нулю. Следовательно, поверхность $Y$ имеет геометрический род $p_{g}=0$.

Поскольку накрытие Галуа $h$ является неразветвленным, то имеет место вложение $\mathbb{Z} / 5 \mathbb{Z} \subset \operatorname{Tors}(Y)$. Чтобы показать, что $\operatorname{Tors}(Y)=\mathbb{Z} / 5 \mathbb{Z}$, достаточно показать, что поверхность $Y_{u(5)}$ односвязна. Более того, легко видеть, что $Y_{u(5)}$ изоморфна некоторой гладкой поверхности в $\mathbb{P}^{3}$. Действительно, выберем однородные координаты $\left(x_{0}: x_{1}: x_{2}\right)$ в $\mathbb{P}^{2}$ так, чтобы $x_{i}=0$ было уравнением прямой $L_{i+2}$. Пусть $\sum a_{i} x_{i}=0$ - уравнение прямой $L_{1}$. Не ограничивая общности, можем предполагать, что накрытие $g_{u(5)}$ ассоциированно с эпиморфизмом $\bar{\varphi}: H_{1}\left(\mathbb{P}^{2} \backslash L, \mathbb{Z}\right) \rightarrow(\mathbb{Z} / 5 \mathbb{Z})^{3}$, заданньм формулами

$$
\bar{\varphi}\left(\lambda_{1}\right)=(0,0,1), \quad \bar{\varphi}\left(\lambda_{2}\right)=(4,4,4), \quad \bar{\varphi}\left(\lambda_{3}\right)=(1,0,0), \quad \bar{\varphi}\left(\lambda_{4}\right)=(0,1,0) .
$$

В этом случае поверхность $Y_{u(5)}$ задается уравнениями

$$
z_{3}^{5}=z_{1}, \quad z_{4}^{5}=z_{2}, \quad z_{5}^{5}=a_{0}+a_{1} z_{1}+a_{2} z_{2}
$$

в неоднородных координатах $\left(z_{1}, z_{2}, \ldots, z_{5}\right)$, где $z_{1}=\frac{x_{1}}{x_{0}}$ и $z_{2}=\frac{x_{2}}{x_{0}}$, и, следовательно, поверхность $Y_{u(5)}$ изоморфна проективному замыканию поверхности в $\mathbb{C}^{3}$, заданной уравнением $z_{5}^{5}=a_{0}+a_{1} z_{3}^{5}+a_{2} z_{4}^{5}$ (cp. [5]).

\section{Список литературы}

1. Barth W., Peters C., Van de Ven A. Compact complex surfaces. Berlin-HeidelbergN.Y.-Tokyo: Springer-Verlag, 1984.

2. Burniat P. Sur les surfaces de genre $P_{12}>0$ // Ann. Pura Appl. 1966. V. 71. № 4. P. 1-24.

3. Campedelli L. Sopra alcuni piani doppi notevoli con curve di diramazione del decimo ordine // Atti Acad. Naz. Lincei. 1932. V. 15. P. 536-542.

4. Dolgachev I. Algebraic surfaces with $q=p_{g}=0$ // Algebraic surfaces. Napoli: Liguori, 1971.

5. Godeaux L. Sur une surface algebrique de genere zero et bigenere deux // Atti. Acad. Naz. Lincei. 1931. V. 14. P. 479-481. 
6. Grauert H., Remmert R. Komplexe Raume // Math. Ann. 1958. V. 136. P. 245-318.

7. Kharlamov V., Kulikov Vik.S. Deformation inequivalent complex conjugated complex structures and applications // Turk. J. Math. 2002. V. 26. P. 1-25.

8. Miyaoka Y. On numerical Campedelli surfaces // Complex analysis and Algebraic geometry. Tokyo: Iwanami Shoten, 1977. P. 113-118.

9. Peters $C$. On certain examples of surfaces with $p_{g}=0 / /$ Nagoya Math. J. 1977. V. 66 . P. 109-120.

10. Mendes Lopes M., Pardini R. A connected component of the moduli space of surfaces with $p_{g}=0$ // Topology. 2001. V. 40. № 5. P. 977-991.

Математический институт им. В. А. Стеклова РАН

Поступило в редакцию

E-mail: kulikov@mi.ras.ru

13.04 .2004 